\title{
Fast Adaptive Digital Pre-Distortion Scheme for Application in Cellular Base Station Power Amplifiers
}

by

Francisca Funmilayo Adaramola, B.Eng.

A thesis submitted to the

Faculty of Graduate and Postdoctoral Affairs

in partial fulfillment of the requirements for the degree of

Master of Applied Science in Electrical Engineering

Ottawa-Carleton Institute for Electrical and Computer Engineering

Department of Systems and Computer Engineering

\author{
Carleton University \\ Ottawa, Ontario \\ June, 2015 \\ (C) Copyright
}

Francisca Funmilayo Adaramola, 2015 
The undersigned hereby recommends to the

Faculty of Graduate and Postdoctoral Affairs acceptance of the thesis

\section{Fast Adaptive Digital Pre-Distortion Scheme for Application in Cellular Base Station Power Amplifiers}

submitted by Francisca Funmilayo Adaramola, B.Eng.

in partial fulfillment of the requirements for the degree of Master of Applied Science in Electrical Engineering

Professor Howard Schwartz, Thesis Co-supervisor

Professor Thomas Kunz, Thesis Co-supervisor

Professor Roshdy Hafez, Chair,

Department of Systems and Computer Engineering

Ottawa-Carleton Institute for Electrical and Computer Engineering

Department of Systems and Computer Engineering

Carleton University

June, 2015 


\section{Abstract}

In a base station, the power amplifier (PA) experiences rapid fluctuations in behavior because of the varying power levels and bandwidth of its excitation signals. High peak-to-average power ratios and non-constant envelopes of wideband signals impose stringent linear and efficiency requirements on the PA required for amplification.

It is necessary to design fast and non-complex digital pre-distorters that are able to achieve and maintain acceptable linearization performance and compensate for the dynamic distortions generated by the PA. This thesis explores an adaptive DPD scheme using multiple model control that combines a switching and a simple adaptation algorithm.

Experimental data are used in evaluating the proposed scheme. The multiple model scheme reduces the transient error response and requires a relatively small number of data samples for identification, switching and adaptation. The scheme is demonstrated to be fast, less complex, and capable of maintaining and achieving the linearity requirement of the PA. 


\section{Acknowledgments}

All thanks to God Almighty for the opportunity, ability and grace to begin and complete this research work. Only by Him, through Him and for Him was this work possible.

Sincere thanks to my supervisors: Howard Schwartz and Thomas Kunz, for believing in me, guiding and leading me patiently and constantly to the completion of this thesis. Thanks to Mark Wyville for the effort, technical support and contribution and to Ericsson for their support that has made this thesis possible.

Gratefully acknowledge the help and support received from staff, professors, friends and colleagues I have met during the period of my study. Appreciation goes to Tosin Olopade and Abubakar Hasssan for your assistance and help in answering all my questions. Thanks to the PhD students in Research Lab 7070 for the fruitful discussions directions and interactions during the work.

Finally, thank you to my family for their encouragement, love and support in all my endeavours. 


\section{Table of Contents}

Abstract $\quad$ iii

Acknowledgments $\quad$ iv

Table of Contents $\quad$ v

List of Tables $\quad$ ix

List of Figures $\quad$ xi

Nomenclature $\quad$ xvi

1 Introduction 1

1.1 Overview . . . . . . . . . . . . . . . . . . 1

1.2 Thesis / Problem Statement . . . . . . . . . . . . . . 5

1.3 Objectives/ Motivation . . . . . . . . . . . . 6

1.4 Contributions ......................... 6

1.5 Organization of the Thesis . . . . . . . . . . . . . 7

2 Background on Power Amplifier $\quad 8$

2.1 Introduction . . . . . . . . . . . . . . . . 8

2.2 PA Nonlinearity and Memory Effect . . . . . . . . . . . 8

2.3 Power Amplifier Characterization . . . . . . . . . . . . . . . 11 
2.3.1 Amplitude and Phase Distortions . . . . . . . . . . . . . . 14

2.3.2 Adjacent Channel Power Ratio . . . . . . . . . . . . . 15

2.3.3 Normalised Mean Square Error . . . . . . . . . . . . . . . . . 16

2.4 PA Modeling . . . . . . . . . . . . . . . . . . . . . 17

2.4.1 Memoryless Nonlinear Models . . . . . . . . . . . . . 18

2.4.2 Memory Nonlinear Models . . . . . . . . . . . . . . . . . . . 19

3 Adaptive Digital Pre-distortion $\quad 24$

3.1 Introduction . . . . . . . . . . . . . . . . . 24

3.2 Pre-distortion . . . . . . . . . . . . . . . . . 25

3.3 Inverse Modeling . . . . . . . . . . . . . . . . . . . . . 28

3.4 Memory Model Based Digital Pre-distortion Schemes . . . . . . . . . 30

3.5 Model Parameters Identification and Estimation Algorithms . . . . . 33

3.5.1 Least Squares Algorithm . . . . . . . . . . . . . . . . . 34

3.5.2 Adaptive Algorithms . . . . . . . . . . . . . . . . . . 35

3.6 Review of Adaptive Digital Pre-distortion Schemes . . . . . . . . . 38

3.7 Improvement to Adaptive DPD Schemes . . . . . . . . . . . . . . . 39

4 Multiple Model Baseband Adaptive Digital Pre-distortion 41

4.1 Introduction . . . . . . . . . . . . . . . . . . . 41

4.2 Data Signals Structure . . . . . . . . . . . . . . . . . . . 41

4.3 Characterization of the PA . . . . . . . . . . . . 43

4.4 Time Delay Estimation and Alignment . . . . . . . . . . . . . 44

4.5 Scaling of Output Data . . . . . . . . . . . . . . . 44

4.6 Data Pre-processing . . . . . . . . . . . . . . . . . . . . 45

4.7 Static Digital Pre-distortion Synthesis . . . . . . . . . . . . . 46

4.8 Adaptive Digital Pre-distortion Synthesis . . . . . . . . . . . . . . . . 49 
4.9 Multiple Model Digital Pre-distortion . . . . . . . . . . . . . . . 51

4.9.1 Offline Parameterization for Model Placement . . . . . . . . 53

4.9.2 Hypothesis Test Switching Algorithm . . . . . . . . . . . . 56

4.9.3 Selected Model Adaptation . . . . . . . . . . . . . . . 59

4.10 Multiple Model Configuration . . . . . . . . . . . . . . . 61

4.11 Scheme Evaluation . . . . . . . . . . . . . . . . . . 62

4.11 .1 Linearization Performance . . . . . . . . . . . . . . 62

4.11.2 Complexity and Speed ................. 64

5 Simulation Results $\quad 66$

5.1 Testbed Equipment Platform Setup . . . . . . . . . . . . 66

5.2 Data Measurement Procedure . . . . . . . . . . . . . . 68

5.3 Time Alignment Results . . . . . . . . . . . . . . . . 73

5.4 PA Characterization . . . . . . . . . . . . . . 74

5.5 Static DPD Simulation . . . . . . . . . . . . . 76

5.5.1 PA and DPD Modeling Simulation . . . . . . . . . . 77

5.5.2 Static DPD Linearization Performance . . . . . . . . . . . . . 80

5.5.3 Effect of Power Levels, Bandwidth and Model Configuration on Linearization Performance . . . . . . . . . . . . . . . 83

5.6 First Order Linear Digital Pre-distortion . . . . . . . . . . . . 86

5.7 Memory Polynomial Based Adaptive DPD Simulation . . . . . . . . . 90

5.8 Multiple Model Switching Algorithm . . . . . . . . . . . . . . . . 94

5.9 Uniform Configuration Multiple Model Performance . . . . . . . . . 95

5.10 Discussion . . . . . . . . . . . . . . . . . . 99

5.11 Non-uniform Configuration Multiple Model Performance . . . . . . . 103

5.12 Discussion . . . . . . . . . . . . . . . . . 107 
5.13 Online Adaptive Parameter Learning . . . . . . . . . . . . . . . . 107

5.14 Number of Models Reduction . . . . . . . . . . . . . . . . . 109

5.15 Summary . . . . . . . . . . . . . . . . . . 109

6 Conclusion and Future Work 111

6.1 Conclusion . . . . . . . . . . . . . . . . . . . . . 111

6.2 Future Work . . . . . . . . . . . . . . . . . . 113

List of References $\quad 115$ 


\section{List of Tables}

4.1 Possible PA and DPD model structure and configuration choice . . . 47

5.1 List of measured input and output multicarrier signals at different ith power levels. The label $i$ is the index of the input signal's power level that specifies the $i t h$ operating condition. The label $\left(D_{i}\right)$ represents the index of the dataset (input and corresponding output) with a carrier size and at a specific $i t h$ power level setting . . . . . . . . . . . . . . 71

5.2 ACPR values of measured datasets for simulation . . . . . . . . . 76

5.3 Modeling accuracy results from the PA and DPD modeling for different configurations using 2C-WCDMA and 4C-WCDMA at the same power level. The number of parameters estimated from each configuration is represented as $K M$. . . . . . . . . . . . . . . . . . . . . . . . 78

5.4 Comparison of the PA and DPD modeling accuracy with a fixed configuration for the 2C-WCDMA signal at different power levels . . . . 80

5.5 Comparison of the ACPR and NMSE of the PA output with a DPD modeled with datasets at different times and the PA output with a static DPD modeled using the signal at only time $t \ldots \ldots$. . . .

5.6 Linearization performance for varying model configurations using the 2C-WCDMA and 4C-WCDMA signal at $-10.5 \mathrm{dBm} \ldots . . . . . .884$ 
5.7 Linearization performance (ACPR in $\mathrm{dBc}$ ) for different configurations using the $4 \mathrm{C}-$ WCDMA signal at varying power levels $\ldots \ldots \ldots . . .85$

5.8 First order linear model adaptive DPD performance for low powered

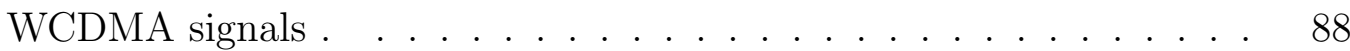

5.9 Comparison of the linearization performance (NMSE \& ACPR) between the LMS (> 50,000 samples) and RLS ( > 500 samples) based adaptive $\mathrm{DPD} \ldots \ldots \ldots \ldots \ldots$. . . . . . . . . . . . . . . 92

5.10 Results of the hypothesis test based switching. 0 indicates that the signal's bandwidth or power level remained constant and a 1 indicates changes in the signal's characteristics . . . . . . . . . . .

5.11 Comparison of the linearization performance (NMSE \& ACPR) of the RLS based ADPD and the multiple model scheme at varying signal

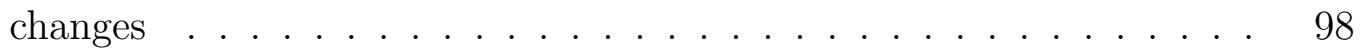

5.12 Choice of model configuration settings chosen for different power level ranges . . . . . . . . . . . . . . . . . . . 104

5.13 Parameter savings estimation . . . . . . . . . . . . . . . 104

5.14 NMSE and ACPR for 2C- and 4C-WCDMA signals exciting the PA at time $\mathrm{t}$ (first occurrence) and at time $\mathrm{t}+\mathrm{q}$ (subsequent occurence) when offline or online learned parameters are used . . . . . . . . . . . 108 


\section{List of Figures}

1.1 Block diagram of a generic wireless transmitter. . . . . . . . . . . 1

1.2 Input-Output power relationship of a PA. Adapted from [1] . . . . 3

2.1 Input and output signal power spectrum showing spectral regrowth after amplification by the $\mathrm{PA} \ldots \ldots \ldots \ldots$

2.2 Input-Output power relationship of (a) memoryless nonlinear PA and (b) memory nonlinear PA . . . . . . . . . . . . . . . 10

2.3 Output spectrum for a one-tone characterization. Adapted from [1] . 13

2.4 Output frequency spectrum from a two-tone characterization. . . . . 14

$2.5 \mathrm{AM} / \mathrm{AM}$ and $\mathrm{AM} / \mathrm{PM}$ characteristic plots for a PA. . . . . . 15

2.6 PA as a black box device under test . . . . . . . . . . . . 17

2.7 Twin box models . . . . . . . . . . . . . . . . . . . . . . . 20

2.8 Wiener-Hammerstein Model . . . . . . . . . . . . . . . . . . . . . 21

2.9 Block diagram of the memory polynomial model . . . . . . . . . . . 22

3.1 Pre-distortion scheme . . . . . . . . . . . . . . . . . 25

3.2 Diagrammatic representation of digital pre-distortion $\ldots \ldots \ldots . .27$

3.3 Model based classification of digital pre-distortion schemes . . . . . 27

3.4 Inverse control methods . . . . . . . . . . . . . . . . . . . . . 29

3.5 Direct learning architecture . . . . . . . . . . . . . . . . . . . . . . 29

3.6 Indirect learning architecture $\ldots \ldots \ldots \ldots \ldots$ 
3.7 Block diagram of a static DPD scheme . . . . . . . . . . . . . 31

3.8 Block diagram of a polynomial-based adaptive DPD . . . . . . . . . . 32

3.9 Block diagram of a gain-based LUT adaptive DPD. Adapted from [2] 32

4.1 Input and output PA data collection . . . . . . . . . . . . . . 42

4.2 Flowchart for the static DPD implementation . . . . . . . . . 46

4.3 Flowchart of an adaptive DPD implementation . . . . . . . . . . 49

4.4 Block diagram of the multiple model scheme . . . . . . . . . . . . . . 51

4.5 Multiple model DPD parameters addressing . . . . . . . . . . . . 52

4.6 Characteristic plot showing the operation of the PA over $i$ distinct operating conditions . . . . . . . . . . . . . . . 54

4.7 DPD scheme parameter initialization . . . . . . . . . . 55

4.8 Model extraction . . . . . . . . . . . . . . . . . . . 55

4.9 Flowchart of the multiple model scheme . . . . . . . . . . . . . . 60

5.1 Block diagram of the experimental platform setup . . . . . . . . . 67

5.2 Transfer characteristics of the driver amplifier in cascade with the PA in the testbed setup . . . . . . . . . . . . . . . . . 68

5.3 Changing input signals experienced by a PA over time . . . . . . . 69

5.4 PSD plots of measured input and output (a) two-carrier WCDMA and (b) four-carrier WCDMA signals for various amplitude settings (legends correspond to $i t h$ values in Table 5.1) . . . . . . . . . . . . . 72

5.5 The GUI of the AMPS software for time delay estimation and align-

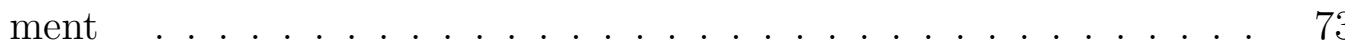

5.6 Comparison between the time domain plot of (a) the original measured input and the aligned input (b) the original measured output and the aligned output and (c) the aligned input and the output signal of a 2C-WCDMA signal . . . . . . . . . . . . . 
5.7 AM/AM and AM/PM response when the PA is excited by a twocarrier WCDMA signal at an input power level of (a) $-10.5 \mathrm{dBm}$ and

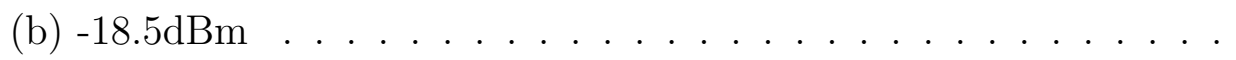

5.8 $\mathrm{AM} / \mathrm{AM}$ and $\mathrm{AM} / \mathrm{PM}$ response when the PA is excited by a fourcarrier WCDMA signal at an input power level of (a) $-10.5 \mathrm{dBm}$ and (b) $-18.5 \mathrm{dBm} \ldots \ldots \ldots \ldots \ldots \ldots \ldots \ldots$

5.9 NMSE values compared to the number of parameters used to model a PA and DPD for a two-carrier(blue) and a four-carrier (red) WCDMA signal at an $i t h$ power level . . . . . . . . . . . . . . . . . . . 79

5.10 Input signal changes experienced by a PA at time $t, t+1$, and $t+2 \ldots 81$

5.11 Linearization performance of a static DP for a PA excited by (a)$10.5 \mathrm{dBm} 4 \mathrm{C}-\mathrm{WCDMA}$ at $t$ (b) $-18.5 \mathrm{dBm} 2 \mathrm{C}-\mathrm{WCDMA}$ at $t+1$ (c) $-10.5 \mathrm{dBm} 2 \mathrm{C}-\mathrm{WCDMA} t+2 \ldots \ldots \ldots \ldots$

5.12 Comparison of the linearization performance of a static DPD with configuration $K=5, M=3$, for a PA excited by WCDMA signals at the same power level but different bandwidths (a)-10.5dBm 2CWCDMA (b)-10.5dBm 4C-WCDMA . . . . . . . . . . .

5.13 Comparison of the linearization performance of a static DPD with configuration $K=3, M=2$ and $K=5, M=3$, at $-10.5 \mathrm{dBm}$ (high powered signal) for (a) a 2C-WCDMA signal and (b) a 4C-WCDMA

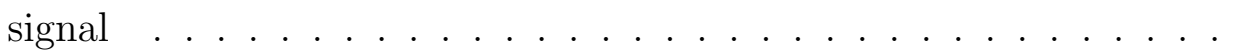

5.14 Comparison of the linearization performance of a static DPD with configuration $K=3, M=2$ and $K=5, M=3$, at $-17.5 \mathrm{dBm}$ (low powered signal) for (a) a 2C-WCDMA signal and (b) a 4C-WCDMA signal. . . . . . . . . . . . . . . . . . . 85

5.15 Block diagram of a first order linear adaptive DPD . . . . . . . . . 86 
5.16 Time plot of the original output and estimated output of a modeled PA for a (a) two-carrier WCDMA signal (b) four-carrier WCDMA signal

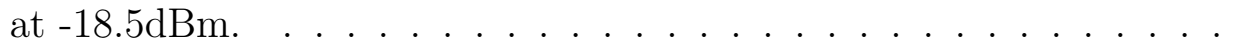

5.17 PSD plots of the original input, the original output before the DPD and the simulated DPD+PA output of a low powered (a) two-carrier WCDMA signal and (b) four-carrier WCDMA signal using the first order linear model-based ADPD . . . . . . . . . . . . . . . .

5.18 PSD plots showing linearization performance of the first order linear model ADPD for a high powered 4C-WCDMA signal. . . . . . . . .

5.19 Transient response of an LMS based adaptive DPD for input signal changes with respect to time . . . . . . . . . . .

5.20 PSD plots of the original input, the original output before DPD, the simulated LMS based DPD+PA output and the simulated RLS based DPD+PA output with 1000 samples from a pure batch of high powered (a) two-carrier WCDMA signal (b) four-carrier WCDMA signal . . .

5.21 Comparison of the linearization performance of the multiple model and LMS based ADPD for low powered (a) two-carrier WCDMA signal and (b) four-carrier WCDMA signal. . . . . . . . . . . . . . .

5.22 Comparison of the linearization performance of the multiple model and LMS based ADPD for high powered (a) two-carrier WCDMA signal and (b) four-carrier WCDMA signal. . . . . . . . . . . . . .

5.23 Measured PA output spectra showing: the measured input (blue), measured output without a DPD (red), output with the LMS based adaptive pre-distorter (pink), RLS based adaptive pre-distorter (black), and LMS based multiple model (green) for signal change to (a) $-17.5 \mathrm{dBm}$ two-carrier WCDMA signal, (b) -17.5dBm four-carrier WCDMA signal. 100 
5.24 Measured PA output spectra showing: the measured input (blue), measured output without a DPD (red), output with the LMS based adaptive ADPD (pink), RLS based adaptive ADPD (black), and proposed multiple model scheme (green) for (a) -10.5dBm two-carrier WCDMA signal, (b) -10.5dBm four-carrier WCDMA signal. . . . . . . . . . . 101

5.25 Comparison of the linearization performance of the multiple model scheme at different configurations with low powered signals for (a) $2 \mathrm{C}$ WCDMA and (b) 4C-WCDMA . . . . . . . . . . . 105

5.26 Comparison of the linearization performance of the multiple model scheme at different configurations with high powered signals for (a)

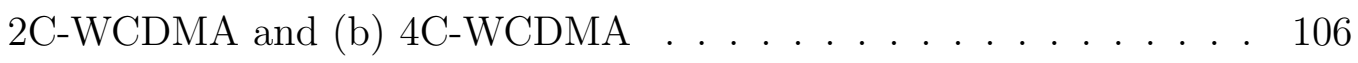




\section{Nomenclature}

\begin{tabular}{ll}
\hline Acronyms & Definition \\
\hline \hline 1dB & 1dB Compression Point \\
ACPR & Adjacent Channel Power Ratio \\
ADPD & Adaptive Digital Pre-distortion \\
AM/AM & Amplitude-Amplitude Distortion \\
AM/PM & Amplitude-Phase Distortion \\
ANN & Artificial Neural Network \\
CALLUM & Combined Analogue Locked Loop Universal Modulator \\
CW & Continuous Wave \\
dB & Decibels \\
DUT & Device Under Test \\
DPD & Digital Pre-distortion \\
DSP & Digital Signal Processing \\
EER & Envelope Elimination and Restoration \\
GMP & Generalized Memory Polynomial \\
LINC & Linear Amplification with Nonlinear Components \\
IMD & Intermodulation Distortion \\
LMS & Least Mean Squares \\
\hline & \\
\hline
\end{tabular}




\begin{tabular}{ll}
\hline Symbols & Definition \\
\hline \hline LS & Least Squares \\
LTE & Long Term Evolution \\
LTI & Linear Time-Invariant \\
LUT & Look-Up Table \\
MM & Multiple Model \\
MP & Memory Polynomial \\
NARMA & Nonlinear Auto-regressive Moving Average \\
NMSE & Normalised Mean Square Error \\
OFDM & Orthogonal Frequency Division Multiplexing \\
PA & Power Amplifier \\
PAPR & Peak Average Power Ratio \\
PD & Pre-distortion \\
RF & Radio Frequency \\
RLS & Recursive Least Squares \\
SSG & Small Signal Gain \\
WCDMA & Wideband Code Division Multiple Access \\
\hline
\end{tabular}




\section{Chapter 1}

\section{Introduction}

\subsection{Overview}

Wireless communication technology has been used for decades to transmit information such as voice, video, and data from one point to another. The transmitter component of the system illustrated in Figure 1.1 comprises of signal processors, modulators, digital-to-analog converters, and power amplifiers (PA). These devices process the input signal and make it suitable for transmission. The transmitter component can be found in the base station of a cellular network.

At present, users of wireless technology require increased data rates and wider bandwidths. These requirements together with the limited frequency spectrum have

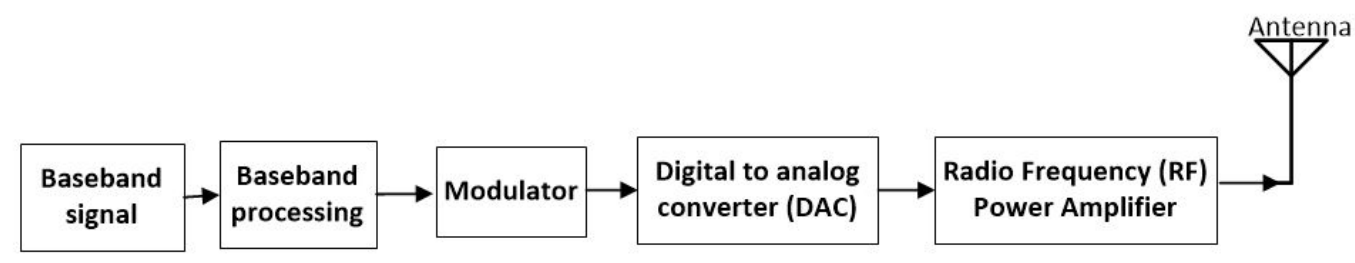

Figure 1.1: Block diagram of a generic wireless transmitter. 
resulted in the development of wireless systems with higher spectral efficiencies. Multiple access techniques produce modern wideband modulation signals capable of providing the functional requirements of modern wireless systems [3]. Examples of these multiple access techniques are Wideband Code Division Multiple Access (WCDMA), Orthogonal Frequency-Division Multiplexing (OFDM), and Long Term Evolution (LTE).

Radio-frequency (RF) PAs amplify information signals desired for transmission and consume more power than other components of the wireless system. PAs can exhibit undesirable behavior and adverse interactions with information signals [4] [5] making them the major source of nonlinearity in a wireless system. The transmitted power of a signal remains confined within its specifed bandwidth when a PA behaves linearly. However, when a PA is nonlinear, spectrum regrowth occurs [6].

The authors in $[7,8]$, reported the effect of the PA nonlinearity on the output spectrum signal. Also, these reports described the memory effect of signals on the PA. There is an inverse relationship between the linearity and efficiency of the PA. Inefficient PAs experience high power losses, which results in system over heating and reduced battery life [3].

Figure 1.2 shows the input-output power relationship of a PA. During low power conditions, there is a linear relationship between the input and output power of the PA. This relationship becomes nonlinear when the input power of the PA exceeds a certain value. This nonlinear response is severe when the input power reaches its saturation point, $\mathrm{P}_{\text {sat }}$, as shown in Figure 1.2.

A potential solution to improve the nonlinear response of the PA is to reduce its input power (back-off power). However, this approach reduces their efficiency [9] as shown in Figure 1.2. A trade-off between the PA linearity and efficiency is therefore inevitable. 


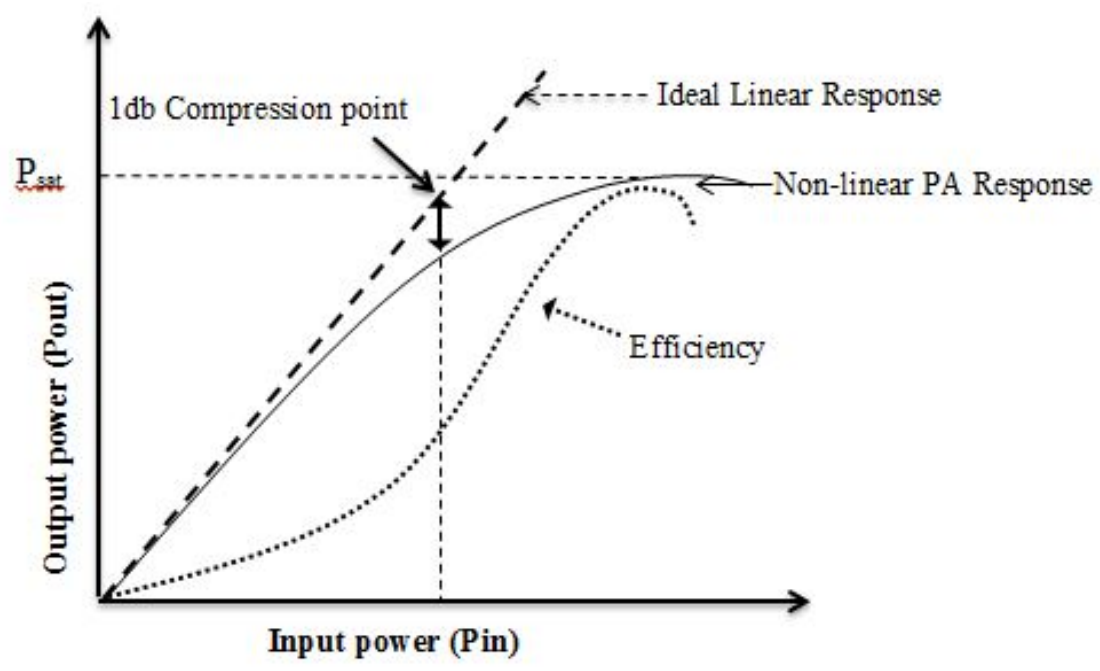

Figure 1.2: Input-Output power relationship of a PA. Adapted from [1]

Wideband modulated signal formats have high peak-to-average power ratios (PAPR) and wide bandwidths. However, high PAPRs drive the PA far into its nonlinear region while wider bandwidth increases the memory effect on the PA. Thus, wideband modulated signals place severe linearity and efficiency requirements on the PA [10]. In the nonlinear region, the PA imposes unwanted frequency spectrum (in-band and out-of-band distortions) to the incoming signal [10]. The unavoidable problem of PA nonlinearity has caused many researchers to explore solutions to an important question: How to maintain the linearity and improve the efficiency of the $P A$ ?

Several linearization techniques have been proposed to improve the performance of PAs. These are feeback, feed-forward, passivity and pre-distortion techniques. Other techniques such as linear amplification with nonlinear components (LINC), combined analogue locked loop universal modulator (CALLUM), envelope elimination and restoration $(\mathrm{EE} \& \mathrm{R})$ are not classified as linearization techniques but they attempt to maintain high efficiency (close to 100\%) [2].

Pre-distortion is a concept which intended to introduce nonlinearity to an input 
signal that can cancel out the intrinsic nonlinearity of the PA [11]. The pre-distorter (PD) can be realised in baseband and implemented using digital signal processing (DSP) techniques. This is termed as Digital Pre-distortion (DPD). DPD compensates for the nonlinearity inherent in a PA and consequently enhances the power efficiency. $[8,12-15]$. The authors in $[8,16,17]$ outlined the advantages the DPD technique has over other techniques. This makes it cost effective and reduces the complexity of its implementation [18] compared to other techniques. The behavior of the digital pre-distorter is modeled as a pseudo-inverse of the PA model. The models of the PA and digital pre-distorter are specified by values referred to as parameters. These parameters identified using mathematical algorithms characterize the model.

Mobile end users communicating with a cellular base station have different needs in terms of type of signals, bandwidth, data rate and power range. As a result, the characteristics of the excitation signal to the PA is rapidly changing over time. Such rapid changes have significant impact on the PA behavior. The characteristics of the signal relates to its power range and bandwidth. The severity of the nonlinearity imposed on signals with higher average power levels is greater compared to signals with lower average levels. Other factors such as aging, temperature changes, supply voltage variations, thermal stress, drift in bias, and frequency changes can also have significant effect on the PA characteristics [17, 19].

Consequently, the digital pre-distorter must continuously compensate for the PA defects to maintain an acceptable linearization performance. Static DPD implementations fall short of the varying changes that could occur during the operation of the PA. Schemes that make use of one-time parameter estimation algorithms such as Least Squares (LS) cannot offer real-time implementation of DPD. Adaptive digital pre-distortion (ADPD) offers a solution for real-time implementation. ADPD can employ the use of adaptive algorithms for real-time estimation of DPD parameters 
and continuously compensate for the nonlinearity and memory effect of the PA [3].

Algorithms such as the Least Mean Squares (LMS) and the Recursive Least

Squares (RLS) can be used for identification of the parameters or coefficients of the model. The adaptive schemes update the parameters of the PD on a sample by sample basis. One major drawback of conventional ADPD schemes is the large transient error experienced in the event of parameter changes [20]. As a result, the overall stability of the system is affected. A comparative study of other linearization techniques [21] shows that they are either unsuitable or have restricted application for PA linearization.

The work presented in this thesis combines multiple model adaptive control and a fast switching algorithm to improve the transient response of the PAs linearized output.

\subsection{Thesis / Problem Statement}

Varying loads of incoming voice and data traffic is a common occurrence in a macro radio base station (RBS). Such loads are interpreted to mean varying characteristics in the input signal that requires processing by a radio frequency (RF) power amplifier. The DPD for linearization must be capable of maintaining the linearity requirement and efficiency of the wireless system which is significantly affected by the PA. To address these problems, there is a need to implement cost effective and fast DPD algorithms. 


\subsection{Objectives/ Motivation}

The main purpose of this thesis is to investigate faster adaptation of a digital predistorter to rapid changes in the behavior of a prototype PA that can be used in a cellular base station. The main objective is to maintain linearity requirements and acceptable performance throughout the operation of the PA. The thesis develops a multiple model adaptive scheme for a digital pre-distorter as a potential solution for achieving PA linearity and efficiency in a fast and cost effective manner.

The overall goal is to ensure that the output of the PA at any time, regardless of the operating conditions or input stimulus, gives an amplified version of the in-

put without in-band or out of band distortions (acceptable linearized performance). Therefore, this work will improve the transient response and complexity of the digital pre-distorter for a PA operating over a large dynamic range.

The approach uses an algorithm that allows for fast switching between multiple models. The situation considered in this thesis is a PA in a wireless base station experiencing rapid behavioral changes as a result of changing characteristics in its excitation signal. The power range and bandwidth of the input excitation signal changes rapidly with respect to time. These changes are caused by the needs of the different users of the wireless system at a particular point in time.

\subsection{Contributions}

The contributions presented in this thesis are as follows:

- A scheme for adaptive DPD realisation is proposed for linearization of a PA having a large dynamic range. The large dynamic range is as a result of the rapidly changing input signals exciting the PA over a short period of time. 
- Application of the hypothesis testing switching method with the proposed DPD multiple model to facilitate fast adaptation and maintain linearization performance.

- A comprehensive evaluation of the multiple model scheme using real-time data collected from an experimental platform setup in Ericsson laboratory.

\subsection{Organization of the Thesis}

The work presented in this thesis is organized into six main chapters. The organization of the thesis is as follows:

Chapter 2 provides a summary of the important concepts of nonlinearity, characterization and the problem of accurate PA modeling for DPD linearization.

Chapter 3 provides a background on and review of related work in adaptive DPD. The issues facing current adaptive digital pre-distorters introduces the topic of multiple models for possible improvements.

Chapter 4 gives a comprehensive description of the methodology for conducting the research and evaluating the work presented. It provides a description of the measurement system for characterizing the PA used for conducting the research. It describes the multiple model adaptive pre-distortion scheme proposed for the PA linearization.

Chapter 5 contains all the simulation and experimental results to assess the work carried out in this research.

Chapter 6 concludes the thesis by reviewing the content and contribution of this work. It states the limitations identified and problems encountered providing directions to potential future work on this topic 


\section{Chapter 2}

\section{Background on Power Amplifier}

\section{$2.1 \quad$ Introduction}

In Chapter 1, the broad topic of wireless communications and the inherent behavior of the PA were introduced. As stated earlier, the main goal for this research is to achieve acceptable linearization performance for a PA in a base station experiencing rapid dynamic behavioral changes. This chapter describes the behavior, characteriz ation and modeling of the PA as an introduction to the concept of DPD and its synthesis.

\subsection{PA Nonlinearity and Memory Effect}

When a PA operates in low power conditions, the PA output power is simply a linear function of the input power. As the power increases, the PA output begins to deviate from the supposed ideal linear response (as shown in Figure 1.2). This ideal response is referred to as the small signal gain $(\mathrm{SSG})$. At the $1 \mathrm{~dB}$ compression point of the PA, the SSG decreases by $1 \mathrm{~dB}$ from the normal expected linear gain. This compression point is defined as the point where the amplifier becomes nonlinear, the amplifier gain 
flattens out, and the amplifier becomes saturated. Beyond this point, the severity of the nonlinearity increases and the PA is said to be operating at saturation.

The effect of the PA nonlinearity on excitation signals is the generation of intermodulation distortions (IMDs). Although such interactions can also generate harmonics, which are polynomial functions of the carrier frequency, they can easily be filtered out. IMDs are observed as frequency components which are linear combinations of excitation frequencies [22]. Figure 2.1 shows the input and output power spectrum of a PA.

IMDs can be categorised into in-band and out-of-band distortions [6]. In-band distortion is a measure of the level of distortion that lies within the allocated channel of the signal. Out-of-band distortion interferes with adjacent channels [9] and is the amount of power that can interfere with neighbouring adjacent channels. The overall effect of distortion is the spectral spreading of the signal.

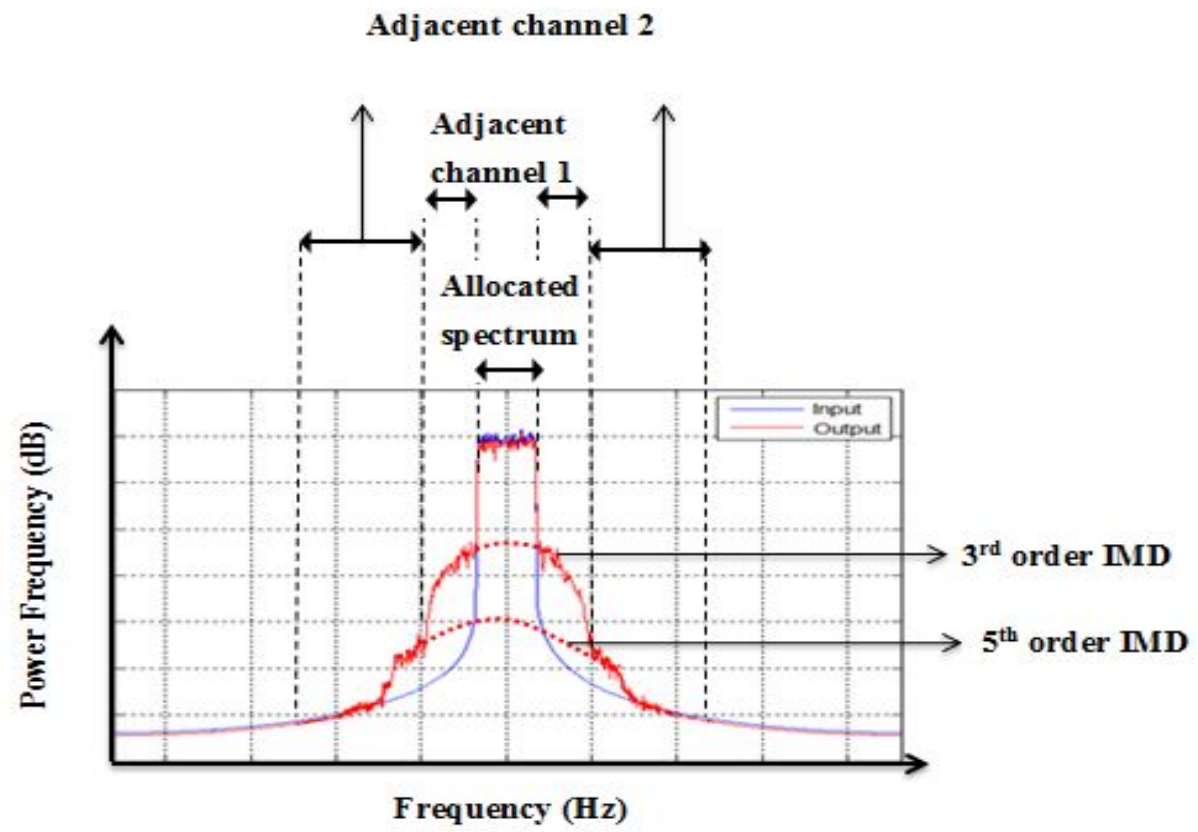

Figure 2.1: Input and output signal power spectrum showing spectral regrowth after amplification by the PA 
Signals generated using advanced and complex modulation schemes, such as the Wideband Code Division Multiple Access (WCDMA) technique, possess high PAPR, drive the power amplifier to saturation and are highly sensitive to PA nonlinearities $[8,23]$. These signals have non-constant envelopes and enable PAs to operate near saturation with improved efficiencies.

In addition to the nonlinearity imposed by the PA on wideband signals, the signals cause the PA to exhibit memory effects. Wider bandwidth signals tend to increase the memory effect exhibited by the PA. In such cases, the response of the PA is affected by the frequency of the input signal and not only its amplitude. Based on this, PA nonlinearity is classified into memoryless nonlinearities and memory-based nonlinearities.

Past inputs affect the present nonlinear effect for memory-based nonlinearities. This means that memory nonlinearities increase the number of parameters for modeling the IMDs. The severity of the memory effect on the PA increases as the number of carriers or bandwidth of the excitation signal increases. The memory effect in a PA usually appears in the input-output power plot, shown in Figure 2.2, as hysteresis and dispersions.

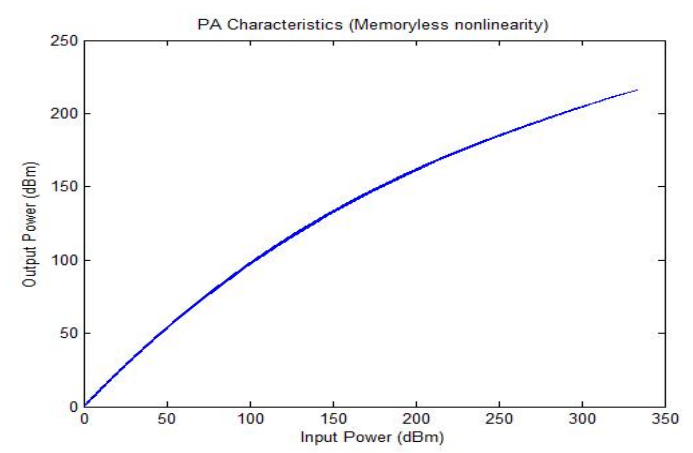

(a) Memoryless PA

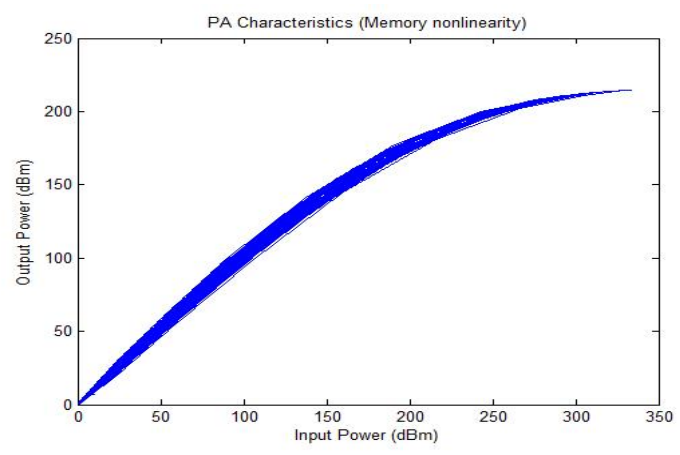

(b) PA with memory

Figure 2.2: Input-Output power relationship of (a) memoryless nonlinear PA and (b) memory nonlinear PA 


\subsection{Power Amplifier Characterization}

It is essential to characterize the distortions caused by the PA in order to adequately compensate for its nonlinearities. The PA is characterized to determine the relationship between the input and the output signal. This characterization specifies the behavior of the PA in terms of the degree of its nonlinearity and the depth of the memory effect it experiences.

Nonlinearity characterization of a PA can be done through testing by sweeping signals across the PA. These tests provide a good understanding of the amplitudeamplitude (AM/AM) distortions, amplitude-phase (AM/PM) distortions, and inband and out-of-band intermodulation distortions exhibited by the PA. Linearity specifications, such as Adjacent Channel Power Ratio (ACPR) and Normalised Mean Square Error (NMSE), are also given to characterize and measure the degree of the unwanted signal generated by the PA.

A third order polynomial given in Equation 2.1 describes the input/output characteristics of a nonlinear PA.

$$
Y=b_{1} X+b_{2} X^{2}+b_{3} X^{3}
$$

where $X$ and $Y$ represent the input and output signals respectively. The $b_{i}$ terms represent the real or complex-valued coefficients. The first order term specifies the gain of the PA and is the only term for a linear PA. The second and third order terms, represent the quadratic and cubic nonlinearities respectively.

For a continuous wave $(\mathrm{CW})$ one-tone characterization, an input signal $X(t)$, can be written as

$$
X(t)=A \sin (w t)
$$


where $A$ and $w$ represent the amplitude and the frequency of the signal respectively. The resulting output of the PA using Equation 2.2 as input gives

$$
Y=b_{1}(A \sin (w t))+b_{2}(A \sin (w t))^{2}+b_{3}(A \sin (w t))^{3}
$$

The expansion of the terms in Equation 2.3 produces Equation 2.4 which can be re-written simply as Equation 2.5

$$
\begin{gathered}
Y=b_{1} A \sin (w t)+b_{2} \frac{A^{2}}{2}-b_{2} \frac{A^{2}}{2} \cos (2 w t)+3 b_{3} \frac{A^{3}}{4} \sin (w t)-b_{3} \frac{A^{3}}{4} \sin (3 w t) \\
Y=a \sin (w t)+b-b \cos (2 w t)+c \sin (w t)-d \sin (3 w t)
\end{gathered}
$$

The second term of Equation 2.3 generates a DC term and a $2^{\text {nd }}$ order harmonic which are the second and third terms of Equation 2.4 respectively. These terms distort the output signal and appear as out-of-band distortions. The third term of Equation 2.3 generates an in-band distortion at the fundamental frequency and a $3^{\text {rd }}$ order harmonic. Figure 2.3 illustrates the output spectrum for the one-tone characterization of the PA. This shows that only odd order nonlinear terms generate in-band distortions [24]. For a two-tone test characterisation, two signals $X_{1}$ and $X_{2}$, written as Equations 2.6 and 2.7 respectively, are used as input to the PA

$$
\begin{aligned}
& X_{1}(t)=A_{1} \sin \left(w_{1} t\right) \\
& X_{2}(t)=A_{2} \sin \left(w_{2} t\right)
\end{aligned}
$$

where $A_{i}^{\prime} s$ and $w_{i}^{\prime} s$ represent the amplitude and the frequency of the input signals respectively. Assuming all $A_{i}{ }^{\prime} s$ are equal, the output of the PA from Equation 2.1 


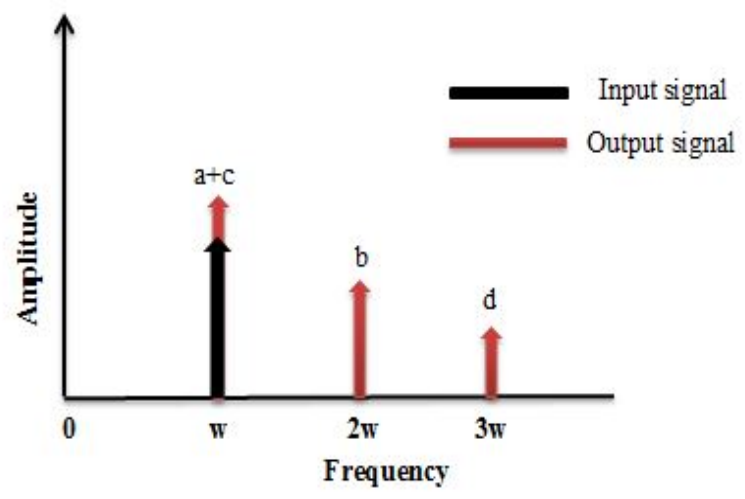

Figure 2.3: Output spectrum for a one-tone characterization. Adapted from [1]

can be written as

$$
\begin{gathered}
Y=b_{1} A\left(\sin \left(w_{1} t\right)+\sin \left(w_{2} t\right)\right)+b_{2} A^{2}\left(1+\cos \left(\left(w_{2}-w_{1}\right) t\right)\right. \\
+\frac{9 b_{3} A^{3}}{4}\left(\sin \left(w_{1} t\right)+\sin \left(w_{2} t\right)\right) \\
+\frac{3 b_{3} A^{3}}{4}\left(\sin \left(\left(2 w_{1}-w_{2}\right) t\right)+\sin \left(\left(2 w_{2}-w_{1}\right) t\right)\right) \\
-b_{2} A^{2}\left(\cos \left(\left(w_{1}+w_{2}\right) t\right)-\frac{b_{3} A^{3}}{4}\left(\sin \left(3 w_{1} t\right)-\sin \left(3 w_{2} t\right)\right)\right. \\
-\frac{3 b_{3} A^{3}}{4}\left(\sin \left(\left(2 w_{1}+w_{2}\right) t\right)-\sin \left(\left(2 w_{1}-w_{2}\right) t\right)\right)
\end{gathered}
$$

Figure 2.4 shows the output frequency components (in-band and out-of-band distortions) generated using the two-tone test. The one-tone characterization is inaccurate as it fails to completely identify the distortions that the PA can impose on a signal $[25,26]$. The two-tone characterization is more accurate than the one-tone characterization. However, it does not capture the full behavior of the PA especially when excited by signals with wider bandwidth. Memory effects exhibited by wideband signals are neglected by these traditional characterizations and this makes it 


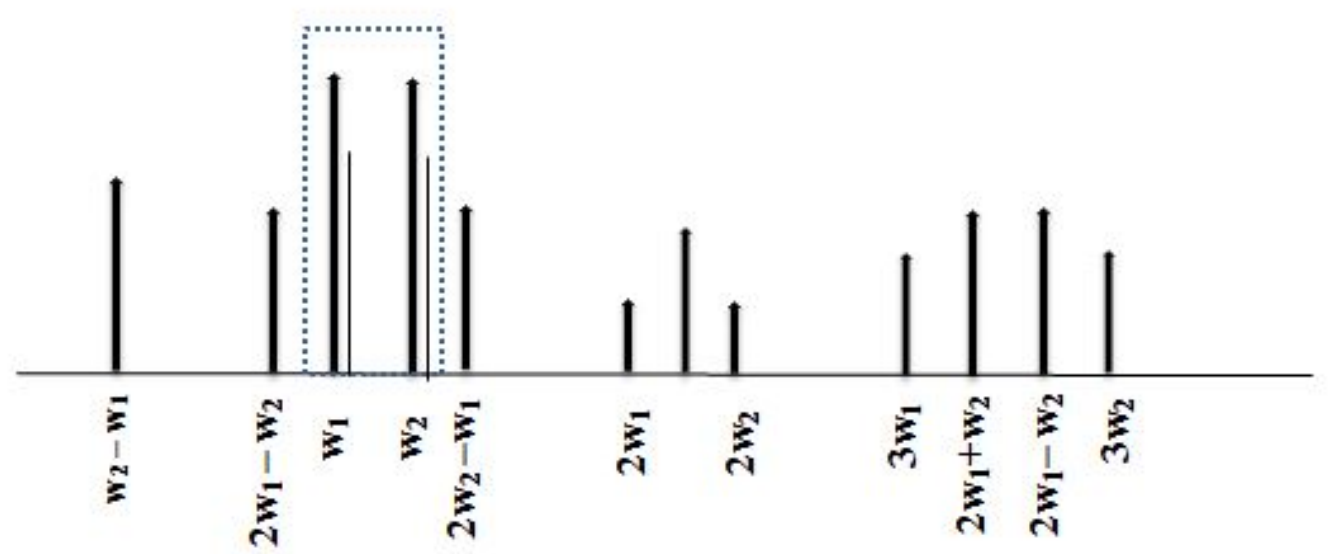

Figure 2.4: Output frequency spectrum from a two-tone characterization.

insufficient for PA characterization. Thus, it is necessary to characterize the PA with signals it will experience in practice that can provide complete modeling of the PA nonlinear behavior.

\subsubsection{Amplitude and Phase Distortions}

The effect of changes in the amplitude of the input on the output amplitude and phase can characterize the nonlinear PA. Two effects that can characterize the nonlinear PA are: 1) the amplitude-amplitude (AM/AM) distortion and 2) the amplitude-phase (AM/PM) distortion. The AM/AM characteristic specifies the changes in the output amplitude power due to changes in the input amplitude power level. The AM/PM is the variation in the phase of the output signal according to the changes in the amplitude of the input signal.

The graphical illustration of these two effects are referred to as the characteristic plots. Figure 2.5 shows the AM/AM and AM/PM characteristic plots of a PA. The 
$\mathrm{x}$ and $\mathrm{y}$ coordinates of the $\mathrm{AM} / \mathrm{AM}$ and $\mathrm{AM} / \mathrm{PM}$ plots can be derived as

$$
\begin{aligned}
& \mathrm{AM}_{x}=20 \log _{10}|x(n)| \\
& \mathrm{AM}_{y}=20 \log _{10}\left\{\frac{|y(n)|}{|x(n)|}\right\} \\
& \mathrm{AP}_{x}=20 \log _{10}|x(n)| \\
& \mathrm{AP}_{y}=\frac{\angle y(n)}{\angle x(n)}
\end{aligned}
$$

where $A M$ and $A P$ are the $\mathrm{AM} / \mathrm{AM}$ and $\mathrm{AM} / \mathrm{PM}$ respectively, $x(n)$ is the input signal, and $y(n)$ is the output signal.
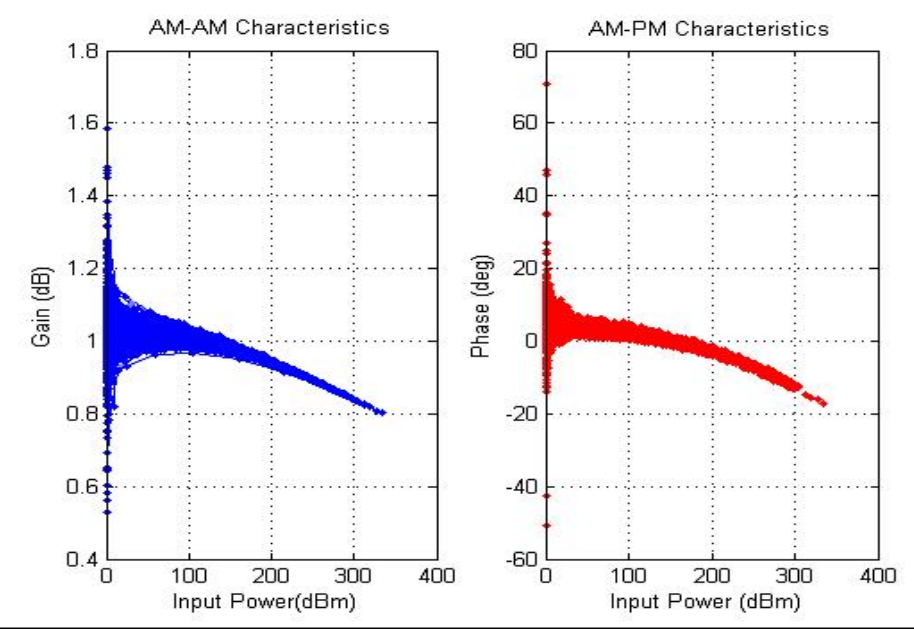

Figure 2.5: $\mathrm{AM} / \mathrm{AM}$ and $\mathrm{AM} / \mathrm{PM}$ characteristic plots for a PA.

\subsubsection{Adjacent Channel Power Ratio}

An important metric to characterize the PA behavior is the Adjacent Channel Power Ratio (ACPR). The ACPR specifies the amount of spectral regrowth in adjacent channels surrounding the main allocated channel. ACPR is the comparison between the power in the adjacent channels and power in the required signal's channel. The ACPR is calculated as 


$$
A C P R=\max _{m=1,2 \ldots} \frac{\int_{(a d j) m}|Y(f)|^{2} d f}{\int_{c h}|Y(f)|^{2} d f}
$$

where $Y(f)$ is the discrete fourier transform of the signal. The integral $\int_{(a d j) m}$ measures the power in each $m$ number of upper and lower adjacent channels close to the main channel. The integral $\int_{c h}$ measures the power in the main channel. The maximum ACPR value from all $m$ adjacent channels is selected as the ACPR measurement.

\subsubsection{Normalised Mean Square Error}

The NMSE compares the measured output signal and the estimated output signal from a model of the PA in the time domain. The NMSE evaluates the in-band distortions of the PA and the accuracy of the estimated time domain output signal of a PA model. The NMSE can be calculated using Equation 2.12 given as

$$
N M S E[d B]=10 \log _{10}\left[\frac{\sum_{n}|y(n)-\tilde{y}(n)|^{2}}{\sum_{n}|y(n)|^{2}}\right]
$$

where $y(n)$ and $\tilde{y}(n)$ represent the measured output and estimated model output signals of the PA respectively.

The typical linearity requirements of a PA specified by the $3 \mathrm{GPP}$ standard [10] for wideband signals are

- $\mathrm{ACPR}$ at $5 \mathrm{MHz}$ must be lesser than $-45 \mathrm{dBc}$ and $-50 \mathrm{dBc}$ at $10 \mathrm{MHz}$.

- NMSE should be lesser than $-35 d B$. 


\subsection{PA Modeling}

Accurate modeling of the distortions which the PA imposes on a signal while interacting with it, is critical for the synthesis of an effective digital pre-distorter. This is because ideally, the pre-distorter should possess complete knowledge and/or estimates of all the distortions and memory effect it is required to compensate for.

Several methods for modeling the behavior of a nonlinear system such as the PA exists. Examples of modeling methods include the physics-based and the system-level models. Physical models require theoretical rules describing the interactions between electronic components that make up the PA [6]. System-level models, otherwise known as behavioural models, help to simplify the problem of modeling, because it requires little knowledge of the PA circuit and hardware functionality.

Behavioral modeling can do without a priori information and only requires the input and output data from the system. The system is taken as a device under test (DUT) represented by a model seen as a black box. A mathematical equation specifies the relationship between the input and output of the black box. These equations used to represent the DUT can define its characteristics in different ways termed as model structures. Figure 2.6 illustrates the PA as a black box.

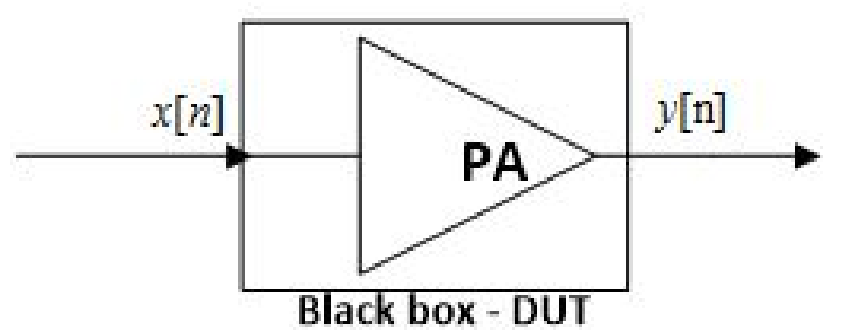

Figure 2.6: PA as a black box device under test 
The two classes of behavioral models for nonlinear systems reported in the literature are memoryless and memory based models. The classification is based on their ability to represent the PA memory effects. It is worth mentioning that no best model exists [14] as modeling depends on factors such as the specific type of PA and its excitation signal. The appropriate model selection is based on the model that is good enough to estimate the behavior of the specific device under test.

\subsubsection{Memoryless Nonlinear Models}

A memoryless nonlinear model has its output as a function of only the present input. They are usually referred to as frequency independent nonlinear models. Current systems employing wideband signals do not use memoryless model structures to represent the PA because they fall short of capturing the memory effects exhibited by wideband signals having wider bandwidth. A few memoryless models exist in the literature such as the Saleh model $[27,28]$, the look-up table model $[2,17]$ and the memoryless polynomial model $[27,29]$.

Saleh [28] proposed a nonlinear model that characterises the behavior of the PA as shown in Equation 2.13

$$
y(n)=\frac{\alpha_{a}|x(n)|}{1+\beta_{a}|x(n)|^{2}} \exp \left\{j \frac{\alpha_{b}|x(n)|^{2}}{1+\beta_{b}|x(n)|^{2}}\right\}
$$

where $\alpha_{a}, \alpha_{b}, \beta_{a}$ and $\beta_{b}$ are the parameters of the model that describe the AM/AM and $\mathrm{AM} / \mathrm{PM}$ distortions and $x(n)$ and $y(n)$ are the input and output of the PA respectively. The memoryless polynomial models approximates the PA behavior as a summation of polynomials written as

$$
y(n)=\sum_{k=1}^{K} b_{k}|x(n)|^{k-1} x(n)
$$


where $x(n)$ and $y(n)$ are the input and output of the PA respectively, $b_{k}$ are the complex valued coefficients and $\mathrm{K}$ is the highest order of the polynomial model. This thesis focuses on wideband signals, which require the use of memory models. Therefore, further information on memoryless models is not provided.

\subsubsection{Memory Nonlinear Models}

Memory effects or dynamic distortions [17] cause the output of the PA to be a function of previous input samples together with current ones. Several memory models with differing levels of complexity have been proposed. These include: Voltera series, twinbox, memory polynomial, and generalised memory polynomial models. Others are: the nonlinear auto-regressive moving average (NARMA) model structure [30], the dynamic deviation reduction (DDR) based Volterra series model [31], artificial neural network (ANN) based model [1] and the look-up table (LUT) model structures. As no perfect model exists, model structure selection is based on a specific application and the power level of operation of the PA.

\section{Volterra Series Model}

The Volterra series is a popular and comprehensive nonlinear model capable of accurately modeling dynamic nonlinear systems $[17,27]$. One major drawback of this complex model is the large number of parameters that are required to be estimated. Equation 2.15 shows the input-output relationship of the Volterra model.

$$
y(n)=\sum_{k=1}^{K} \sum_{i_{1}=0}^{M} \ldots \sum_{i_{k}=0}^{M} h_{k}\left(i_{1}, \ldots, i_{k}\right) \prod_{j=1}^{K} x\left(n-i_{j}\right)
$$

where $h_{k}\left(i_{1}, \ldots, i_{k}\right)$ are the parameters of the model, $K$ is the nonlinearity order and $M$ is the memory depth. The model is well suited for mild nonlinearity. In the face 
of strong nonlinearity, the Volterra model will require a large number of parameters. Other variants of the Volterra series with reduced complexity (number of parameters) and comparable performance have been developed in [13,30-32].

\section{Twin-box Models}

Twin box models are a combination of a linear time-invariant (LTI) system with an impulse response function as Equation 2.16, followed by a memoryless nonlinearity given in Equation 2.17. This model assumes that memory effects are linear and can be decoupled from the nonlinear system [32]. One disadvantage of the Wiener (Figure 2.7(a)) and Hammerstein models (Figure 2.7(b)) is the fact that the output depends nonlinearly on the coefficients. Estimation of coefficients becomes more difficult in the twin-box models compared to models that are linear in the parameters [13].

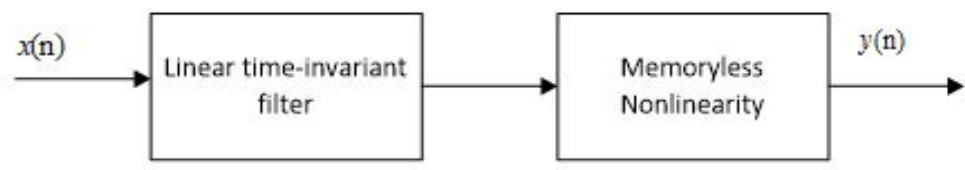

(a) Wiener model

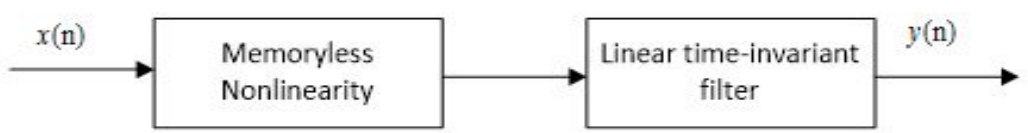

(b) Hammerstein model

Figure 2.7: Twin box models

The combination of the Wiener and Hammerstein models seeks to improve upon 
the undesirability of the twin-box models. The Wiener-Hammerstein model is illustrated in Figure 2.8.

$$
\begin{gathered}
w(n)=\sum_{m=0}^{M} c_{m} x(n-m) \\
y(n)=\sum_{k=1}^{K} b_{k} w(n)|w(n)|^{k-1}
\end{gathered}
$$

where $x(n)$ and $y(n)$ are the input and output of the PA respectively, $b_{k}$ and $c_{m}$ are the coefficients, $K$ is the highest order of the memoryless nonlinear equation and $M$ is the memory length of the impulse response function. In Equation 2.17, the coefficients $c_{m}$ will appear nonlinear.

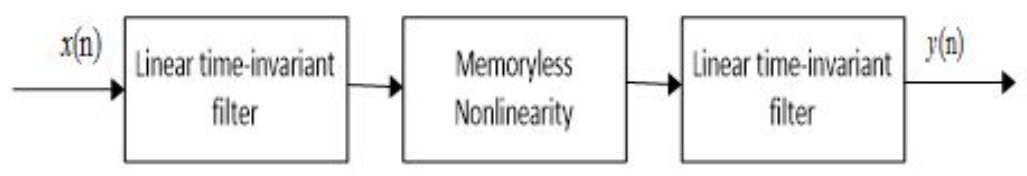

Figure 2.8: Wiener-Hammerstein Model

\section{Memory Polynomial Model}

The memory polynomial (MP) model has been extensively used in the literature, especially in the linearization of power amplifiers with memory $[1,14,17,27]$. It is a special case of the Volterra model proposed by [15] and usually referred to as pruned Volterra series model. It is a simpler form of the Volterra series with a high modeling accuracy capability for a nonlinear system.

The output of this model is linear with respect to the parameters. This makes it possible for the model parameters to be extracted systematically and directly using linear identification algorithms such as the least squares algorithm. Figure 2.9 shows a block diagram representation of the memory polynomial model. The MP model 
structure can be represented in different forms written as

$$
\begin{gathered}
y(n)=\sum_{k=1}^{K} \sum_{m=0}^{M-1} a_{k m} x(n-m)|x(n-m)|^{k-1} \\
y(n)=\sum_{m=0}^{M} \sum_{k=1}^{K} a_{2 k-1, m} x(n-m)|x(n-m)|^{2(k-1)}
\end{gathered}
$$

where $x(n)$ and $y(n)$ are the input and output respectively, $a_{k m}$ and $a_{2 k-1, m}$ are the coefficients, $K$ is the highest nonlinear order and $M$ is the memory length. The form in Equation 2.18 uses even and odd terms $\left(x, x|x|, x|x|^{2}, x|x|^{3} \ldots\right)$. Equation 2.19 uses only odd nonlinear terms $\left(x, x|x|^{2}, x|x|^{4}, x|x|^{6} \ldots\right)$. Including the even-order nonlinear terms can increase modeling accuracy and it permits the use of lower-order polynomials that can offer better numerical accuracy [33].

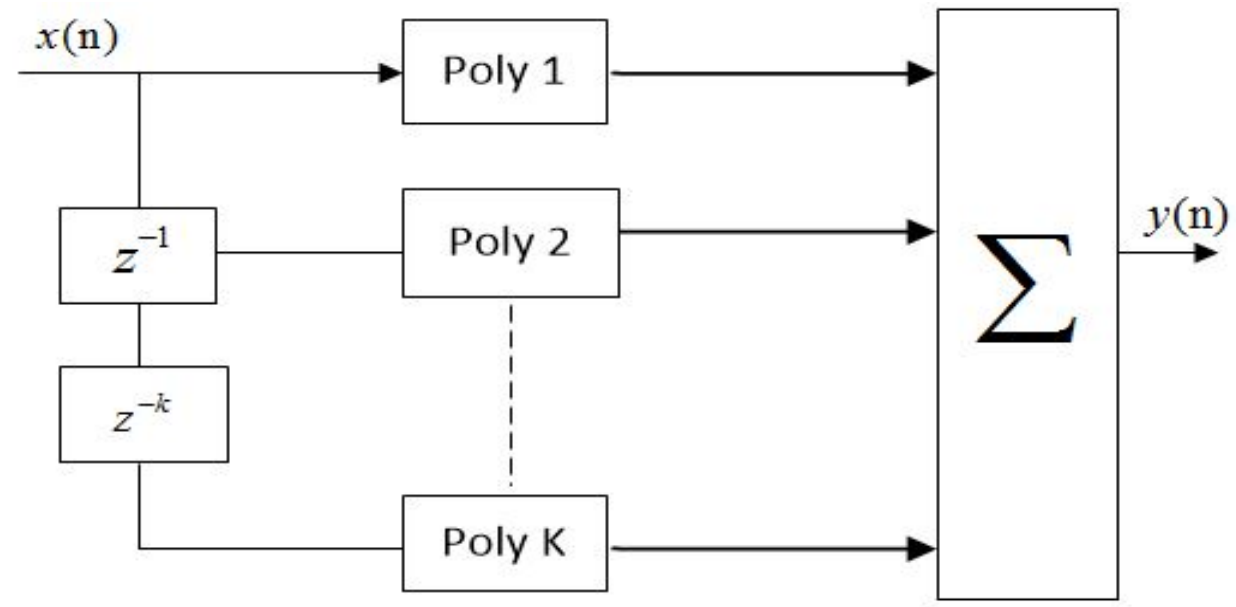

Figure 2.9: Block diagram of the memory polynomial model

A more robust variant of the MP model is the generalized memory polynomial 
(GMP) [13]. The model can be written as

$$
\begin{gathered}
y(n)=\sum_{k=0}^{K_{a}-1} \sum_{l=0}^{L_{a}-1} a_{k l} x(n-l) \mid x(n-l)^{k}+ \\
\sum_{k=1}^{K_{b}} \sum_{l=0}^{L_{b}-1} \sum_{m=1}^{M_{b}} b_{k l m} x(n-l)|x(n-l-m)|^{k}+ \\
\sum_{k=1}^{K_{c}} \sum_{l=0}^{L_{c}-1} \sum_{m=1}^{M_{c}} c_{k l m} x(n-l)|x(n-l+m)|^{k}
\end{gathered}
$$

where $x(n)$ and $y(n)$ are the input and output respectively, $K_{a} L_{a}$ represents the number of coefficients similar to those of the MP model, $K_{b} L_{b} M_{b}$ are the number of coefficients for lagging envelope and signal, and $K_{c} L_{c} M_{c}$ represents the number of coefficients for the signal and leading envelope. The cross-terms, $(x(n)|x(n-1)|, x(n) \mid x(n-$ $2)|, x(n-1)| x(n-2) \ldots)$, can be estimated using linear estimating algorithms and this gives favorable implications for algorithm stability and computational complexity.

This thesis uses the MP model structure for fitting the characteristics of the PA and its inverse from measured input and output. Polynomial models have showed good performance when used with weak or high nonlinear PA [14]. The number of parameters are not as much as the Volterra series model and they can be estimated using linear model identification algorithms. 


\section{Chapter 3}

\section{Adaptive Digital Pre-distortion}

\subsection{Introduction}

Digital pre-distortion is a technique used for the linearization of power amplifiers. It has the capability to improve the linearity and efficiency of PAs. DPD uses the inverse models of power amplifiers to control the output of the PA. This approach is referred to as inverse modeling. When the operating condition of a PA changes, the output of the PA may experience different levels of distortion. Therefore, adaptive DPD can maintain the desired output of the PA.

At present, several adaptive DPD schemes exist. The scheme proposed in this thesis is intended to maintain the linearity of PAs through faster adaptation, re-

duced transient errors and reduced complexity. This chapter reviews the concept of pre-distortion, inverse modeling, adaptive DPD and model identification algorithms. Adaptive DPD schemes are also reviewed. 


\subsection{Pre-distortion}

Pre-distortion is the introduction of distortion to the input signal before its interaction with a PA. A pre-distorter (PD) is a nonlinear block with an inverse characteristic of the PA [6]. The purpose of the PD is to introduce a complementary nonlinearity to an input signal that can cancel out the intrinsic nonlinearity of the PA [11]. Figure 3.1 illustrates the concept of pre-distortion. The pre-distorter is inserted prior to the PA to invert the gain characteristic of the PA. The PD introduces an expansive distortion to the signal which is cancelled by the compressive distortion introduced by the PA.
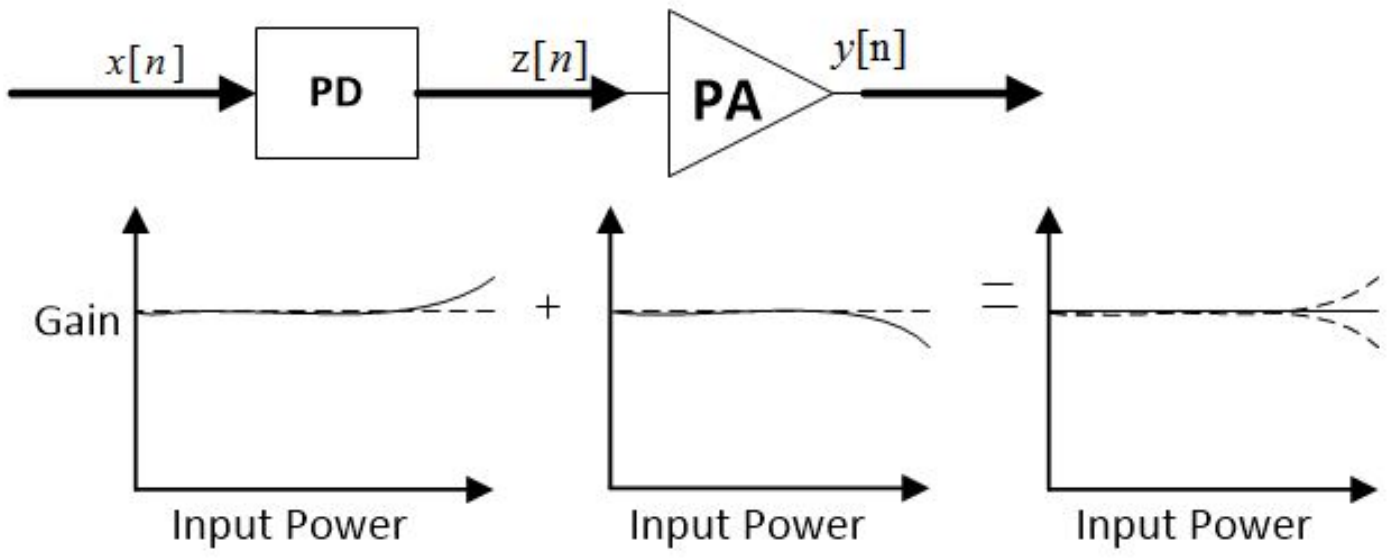

Figure 3.1: Pre-distortion scheme

The PA output $y_{p}$ and the PD output $y_{d}$ can be given as

$$
\begin{aligned}
& y_{p}=x_{p} \cdot G\left(\left|x_{p}\right|\right) \\
& y_{d}=x_{d} \cdot F\left(\left|x_{d}\right|\right)
\end{aligned}
$$

where $G\left(\left|x_{p}\right|\right)$ and $F\left(\left|x_{d}\right|\right)$ denote the AM-AM and AM-PM characteristics of the PA and PD respectively. Whereas, $x_{p}$ and $x_{d}$ represent the input signal of the PA and 
PD respectively. The terms $y_{p}, x_{p}, y_{d}, x_{d}, G\left(\left|x_{p}\right|\right)$ and $F\left(\left|x_{d}\right|\right)$ are all in complex form. The output of the cascade of the PA and PD is given as

$$
y_{p}=x_{d} \cdot F\left(\left|x_{d}\right|\right) \cdot G\left(\left|x_{d} \cdot F\left(\left|x_{d}\right|\right)\right|\right)
$$

Overall, the output of the linearized PA can be written as

$$
y_{p}=A \cdot x_{d}
$$

where $A, y_{p}$ and $x_{d}$ represents the gain, output and input signal of the linearized PA respectively.

The PD can be implemented in analog at intermediate frequency (IF) or in digital form at baseband using digital signal processing (DSP) techniques. The pre-distortion achieved at baseband is referred to as baseband digital pre-distortion (DPD). Implementing pre-distortion with DSP at baseband is usually preferred because it reduces cost, and enjoys flexibility [34], and is better suited for realising adaptive predistorters $[16,19,23]$. The work reported in [23] showed that a digital pre-distorter offered better linearization performance than other analog pre-distorters.

Baseband DPD incorporates both up- and down- conversion. Figure 3.2 shows the representation of a digital pre-distorter scheme. The signal from the PA is downconverted (from RF to digital) before it can be processed by the PD. The output signal of the PD is up-converted (digital to RF) for power amplification. The DPD operates in the digital domain of a general transmitter chain.

The synthesis of the pre-distorter function requires an adequate model structure that models the behavior of the pre-distorter. However, the performance of a digital pre-distorter depends on how well the actual nonlinearity of the PA and its inverse 


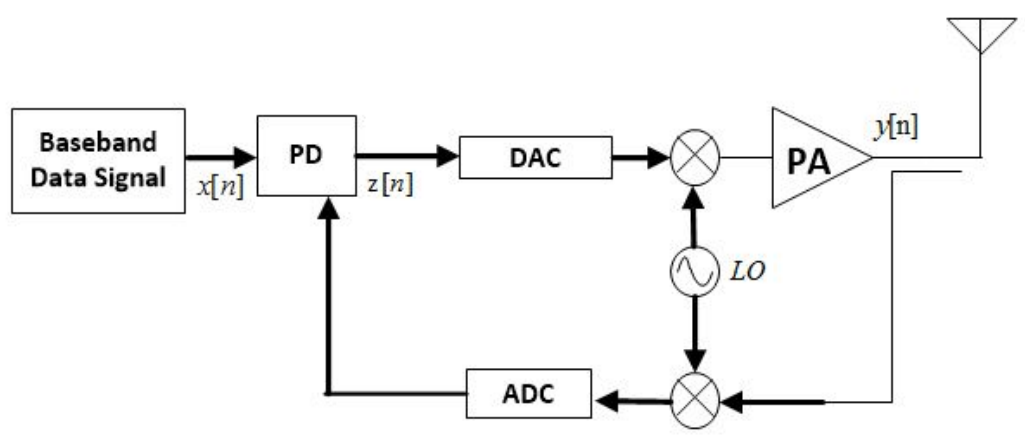

Figure 3.2: Diagrammatic representation of digital pre-distortion

function are modeled to match their true characteristics. The relationship between the input and output of the pre-distorter can be determined using models.

Behavioral models, such as those described in Section 2.4, are used to model the behavior of the digital pre-distorter. DPD schemes can also be classified based on the models used to characterize them. The two classes are: 1) the memoryless model based DPD and 2) memory model based DPD. These are further divided into static and adaptive schemes as shown in Figure 3.3.

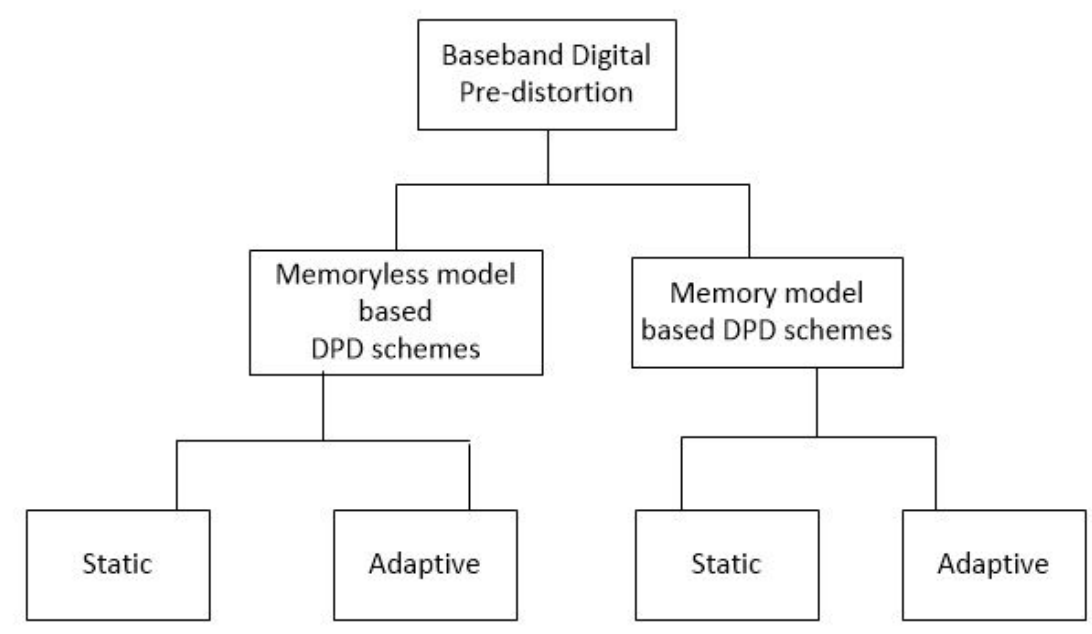

Figure 3.3: Model based classification of digital pre-distortion schemes 
The memory based schemes can capture the memory effects exhibited by the wideband signals while the memoryless schemes cannot capture memory effects. The characteristics of the model specified by the parameters of the model structure can then be estimated using identification algorithms. As mentioned earlier, memory based schemes are considered because wideband signals are used in this thesis.

\subsection{Inverse Modeling}

Inverse modeling is described as the method by which a model uses its inverse to control itself. This approach reduces the difference between the input and output of the system. The inverse of a model can be applied before or after the model to be controlled. Application of the inverse model before and after is referred to as pre-inverse and post-inverse respectively. Figure 3.4 shows the diagrammatic representation of the concept of pre- and post-inverse modeling. The method chosen to estimate the inverse of a system has a large impact on the result of the cascade of the inverse model and the model to be controlled. Inverse modeling is used in DPD for control and linearization of the PA.

One common problem with the pre- and post-inverse methods is finding the inverse model itself. There are different methods for estimating a system's inverse. These include inversion by feedback, analytic inversion, and inversion by system simulation [35]. Inversion by feedback requires that a model that characterises the PA is selected.

The model of the PA is estimated and inverted to generate the inverse model, the PD.

Analytic inversion uses an ideal pre-distorter to obtain an estimate. The PA model is estimated and the PD model is placed in series with the PA. The parameters are estimated based on the error between the input and the output of the PA and PD 


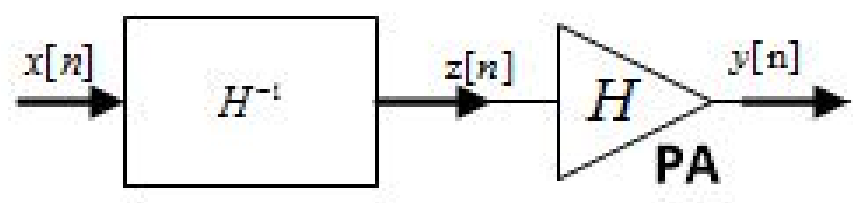

Pre-inverse

(a) Pre-inverse

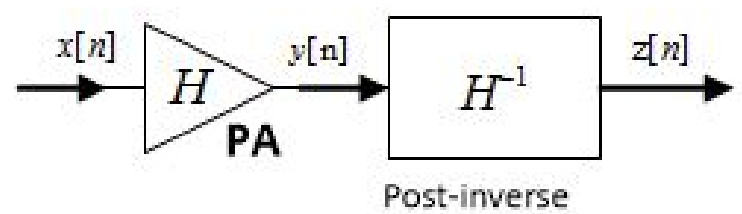

(b) Post-inverse

Figure 3.4: Inverse control methods

combination. This is termed the direct learning architecture (DLA) [15]. The same model structures can be used for the PA and the PD or the PA model structure can be more complex. In DLA, the predistorter is obtained by direct pre-inversion of the PA characteristics. Figure 3.5 illustrates the direct learning architecture.

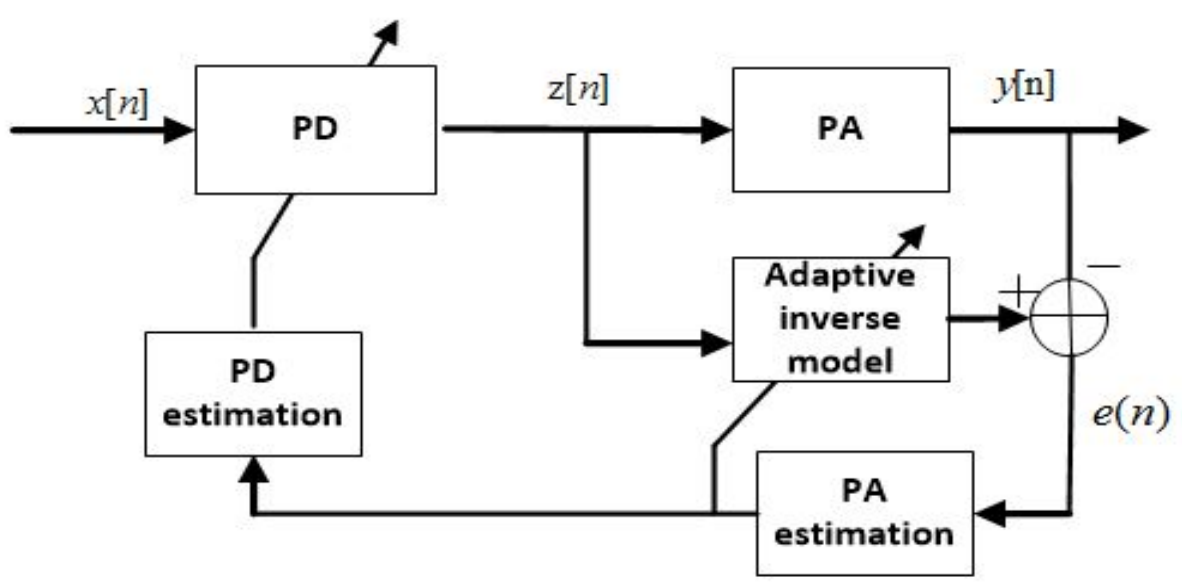

Figure 3.5: Direct learning architecture 
Inversion by system simulation estimates the inverse of the PA directly without a PA model. Input and output data are used directly to identify the inverse model. This is termed as the indirect learning architecture (ILA) [14]. For the ILA, a post-inverse of the nonlinear model is derived and then transferred for use as a pre-distorter. Figure 3.6 illustrates the indirect learning architecture. A PD implemented using the ILA proposed in [14] achieved a robust linearization performance because the memory polynomial structure based pre-distorter was not tied to a particular PA model.

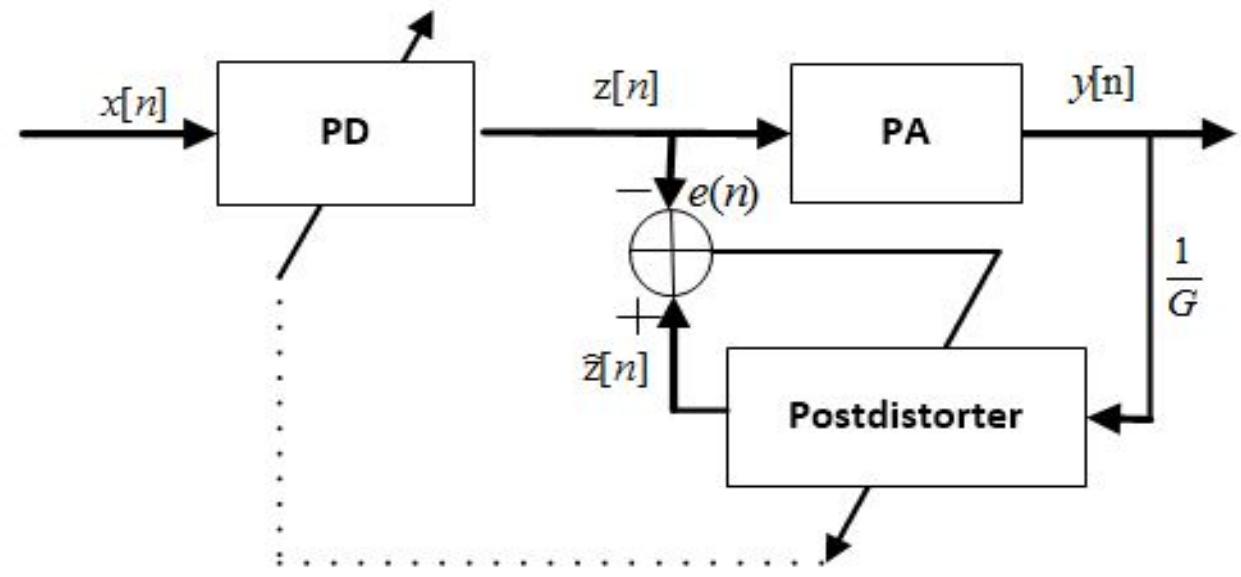

Figure 3.6: Indirect learning architecture

In this thesis, the indirect learning architecture is the inverse modeling method of choice. The basis for this is because it is more commonly used than the direct learning architecture and the inverse can be estimated directly.

\subsection{Memory Model Based Digital Pre-distortion Schemes}

Static memory model based DPDs, sometimes referred to as open-loop DPD architecture, can be suitable for PAs with small dynamic ranges. They are effective when the 
PA's input-output characteristics are nonvarying. Figure 3.7 shows the diagrammatic representation of the static pre-distorter scheme. The characteristics of the PA and DPD can be estimated using an estimation algorithm such as the Least Squares (LS) algorithm. This estimation is a one-time estimation done offline.

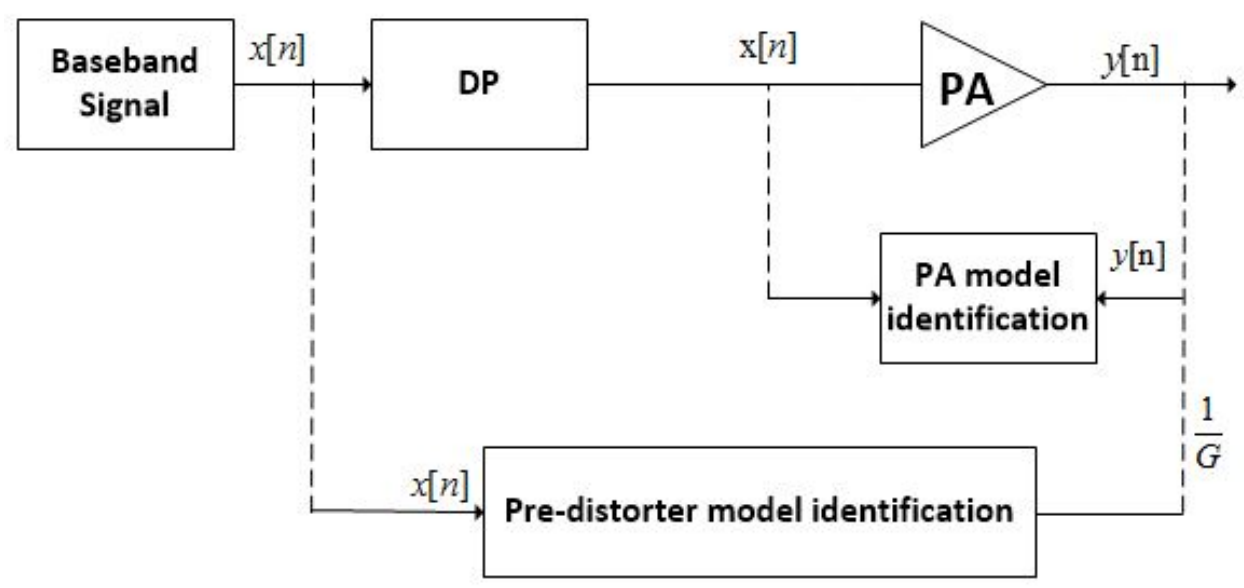

Figure 3.7: Block diagram of a static DPD scheme

The nonlinear behaviour of the PA depends on the statistics of its input signal $[5,36]$. The AM/AM and AM/PM distortions are functions of the input signal and different levels of distortions can be obtained for different excitation signals. Thus, static DPD cannot compensate for the changing characteristics exhibited by the PA.

Adaptive DPDs can compensate for the nonlinearities of a PA in real time [34] and continuously update the coefficient of the DPD. They are capable of tracking possible changes in the PA behavior by getting feedback from the PA's output. The adaptation is based on the difference between the desired output and the PA's actual output.

Adaptive DPD schemes can employ adaptive filtering algorithms that estimate the pre-distorters coefficient .Adaptation can also be performed through the use of a look-up table (LUT) [37,38]. A complex polynomial generates and updates the entries of the LUT and the parameters of the PD model are drawn and updated from the 
LUT. For the former, polynomial-based adaptation algorithms such as Least Mean Squares (LMS) and Recursive Least Squares (RLS), are used for the identification and continuous update of the model parameters. Figure 3.8 shows the generic polynomialbased adaptive scheme for PA linearization. The LUT-based adaptive scheme is very

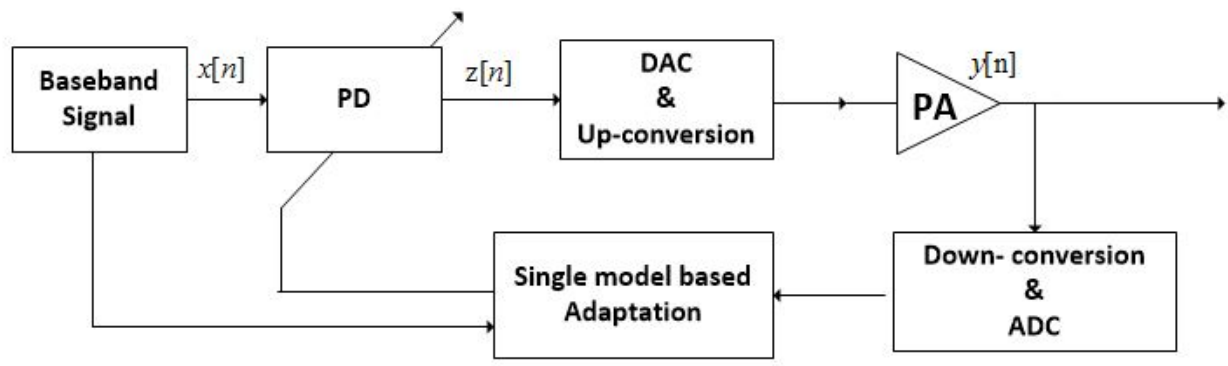

Figure 3.8: Block diagram of a polynomial-based adaptive DPD

flexible and has high accuracy. An increase in the number of LUT entries increases the accuracy of the estimated model. However, the complexity of the scheme increases as the number of LUT entries increases. Thus, performance trade-offs exist with the LUT-based adaptive DPD schemes. Figure 3.9 shows a gain-based LUT adaptive DPD scheme.

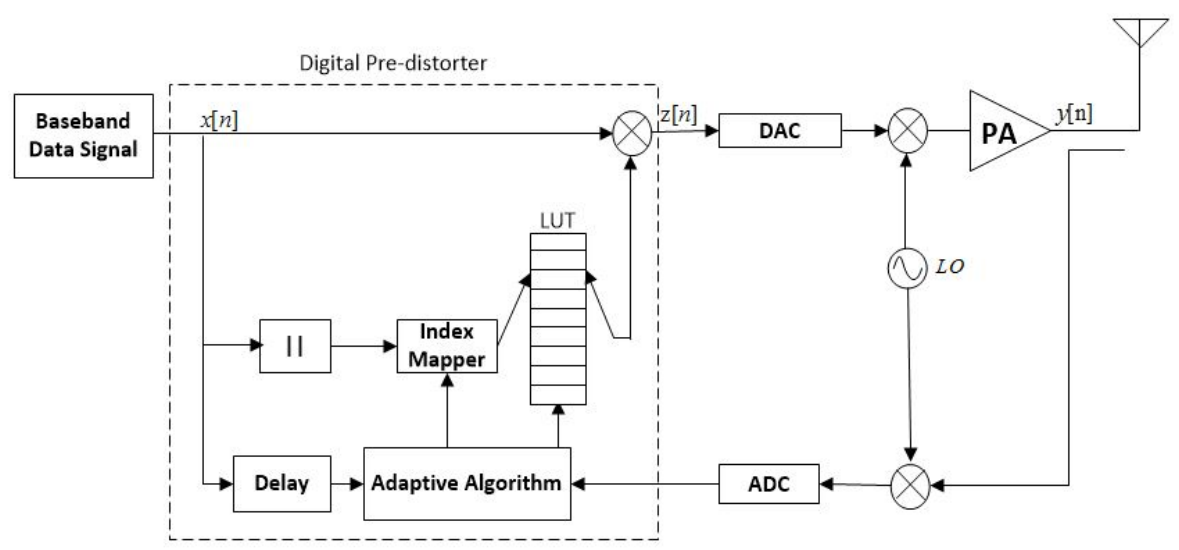

Figure 3.9: Block diagram of a gain-based LUT adaptive DPD. Adapted from [2] 
For all adaptive algorithms, coefficients of the PD model are updated by comparison between the original excitation signal at the PDs input, and the PA output. This is carried out on a sample-by-sample basis and the size of the estimation error is monitored to continuously minimize it. The error is used in the adaptation algorithm to control the system. A comparison of the polynomial and LUT-based adaptations reported in [19] showed that the polynomial-based adaptation algorithms provided better performance in terms of convergence time and error than the LUT-based adaptation. This work focuses on the use of polynomial-based memory model adaptive pre-distortion.

\subsection{Model Parameters Identification and Estima- tion Algorithms}

Model parameters are extracted using identification algorithms that make use of the input data and the corresponding output data from the system to be identified. The accuracy of the estimated output of the model depends on the model structure chosen and the parameter extraction algorithm used. Most of these models are approximates of the complex Volterra series model and this makes the quality of the behavioral model depend on the parameter extraction process rather than the model structure [39]. The main algorithms for extracting model parameters obtained from real time

data measurements are: least squares (LS), least mean squares (LMS), and recursive least squares(RLS). 


\subsubsection{Least Squares Algorithm}

The least squares (LS) algorithm is used for a one-time parameter estimation of a model. It finds the best set of parameters for a model using a given set of data points by minimizing the sum of the squares of the residuals between the true system output and the model's output estimates. Equation 3.5 describes the model structure associated with the memory polynomial model of a nonlinear system.

$$
y(n)=\sum_{k=1}^{K} \sum_{m=0}^{M-1} a_{k m} x(n-m)|x(n-m)|^{k-1}
$$

where $x(n)$ and $y(n)$ are vectors containing the input and output data samples, $M$ is the memory depth and $K$ is the nonlinear order. The variable $n$ denotes the sample index Equation 3.5 can be written as:

$$
\begin{aligned}
& y(n)=\phi(n) \theta \\
& \phi(n)=\left[\begin{array}{ccccccc}
x_{10} & \cdots & x_{1(M-1)} & \cdots & x_{11}\left|x_{11}\right|^{K-1} & \cdots & x_{1(M-1)}\left|x_{1(M-1)}\right|^{K-1} \\
x_{20} & \cdots & x_{2(M-1)} & \cdots & x_{21}\left|x_{21}\right|^{K-1} & \cdots & x_{2(M-1)}\left|x_{2(M-1)}\right|^{K-1} \\
\vdots & \vdots & \vdots & \cdots & \vdots & \vdots & \vdots \\
x_{N 0} & \cdots & x_{N(M-1)} & \cdots & x_{N 1}\left|x_{N 1}\right|^{K-1} & \cdots & x_{N(M-1)}\left|x_{N(M-1)}\right|^{K-1}
\end{array}\right] \\
& \theta^{T}=\left[\begin{array}{llllllllll}
a_{10} & \cdots & a_{1(M-1)} & a_{20} & \cdots & a_{2(M-1)} & \cdots & a_{K 0} & \cdots & a_{K(M-1)}
\end{array}\right]
\end{aligned}
$$

where $\phi(n)$ is the regression matrix formed from all the present and past inputs written as Equation 3.7 and $\theta$ is the vector containing unknown complex-valued parameters of the model written as Equation 3.8. 
The least squares solution gives the estimate of the model parameters and is computed as

$$
\tilde{\theta}=\phi^{+}(n) y(n)
$$

where $\phi^{+}(n)$ is the pseudoinverse of $\phi(n)$ given as

$$
\phi^{+}(n)=\left(\phi^{H}(n) \phi(n)\right)^{-1} \phi^{H}(n)
$$

$\mathrm{H}$ represents the hermitian or complex conjugate transpose. The estimate of the model output is computed as

$$
\tilde{y}(n)=\phi(n) \tilde{\theta}
$$

The model parameters estimated are chosen to minimize the prediction error $e(n)$ between the actual output $y(n)$ and the estimated output $\tilde{y}(n)$ given as

$$
e(n)=y(n)-\tilde{y}(n)
$$

The accuracy of the model and the estimated parameters associated with the estimation is evaluated using the Normalised Mean Square Error (NMSE) metric computed as Equation 2.12 in Section 2.3.3. The NMSE value determines to what extent the model fits the data. A small NMSE value is an indication of a good estimate.

\subsubsection{Adaptive Algorithms}

Stochastic gradient algorithms (LMS) and sample-by-sample based adaptive estimation algorithms (RLS) are examples of adaptive algorithms employed for adaptive digital control. Each of these algorithms differ in performance, convergence speed 
and computation complexity. The performance, accuracy and stability of these algorithms affect the overall performance of the adaptive DPD scheme.

\section{Least Mean Squares (LMS)}

The parameters of a model given as Equation 3.5 and 3.6 can be determined online using the least mean squares (LMS) algorithm. The model parameters, $\theta$ (Equation 3.8) are continuously adjusted to minimize $e(n)$ given as

$$
e(n)=y(n)-\tilde{y}(n)
$$

where $y(n)$ and $\tilde{y}(n)$ are the actual and estimated output respectively and $n$ is the sample index. $\tilde{y}(n)$ is given as

$$
\tilde{y}(n)=\phi(n) \tilde{\theta}_{n}
$$

The algorithm computes the current parameters using:

$$
\tilde{\theta}_{n+1}=\tilde{\theta}_{n}+\left(\mu * \phi^{T}(n) * e(n)\right)
$$

where $\phi(n)$ is the regression matrix, $\tilde{\theta}_{n}$ are the parameters using previous $n$ samples, $\tilde{\theta}_{n+1}$ are related to current $n+1$ samples, $\mu$ is the step size and $(.)^{T}$ denotes the transpose operator. The current parameter, $\tilde{\theta}_{n+1}$, is based on previously estimated parameters, $\tilde{\theta}_{n}$, and $e(n)$. The step size $\mu$ is chosen such that

$$
0<\mu<\frac{2}{\lambda_{\max }}
$$

where $\lambda_{\max }$ is the maximum eigenvalue of the covariance matrix $\rho$ derived as

$$
\rho=E\left[\phi(n) \phi^{T}(n)\right]
$$


where $\phi(n)$ is the regressor vector, $E[$.$] represents the mean and \lambda_{\min }$ is the minimum eigenvalue of the covariance matrix. The condition number of the covariance matrix tells how good an estimation is. A small condition number indicates a well conditioned system and a good estimate can be determined. The illconditioning of this matrix will generate a bad model estimate. The condition number is defined as

$$
\subset=\frac{\lambda_{\max }(\rho)}{\lambda_{\min }(\rho)}
$$

\section{Recursive Least Squares (RLS)}

The set of Equations 3.19 to 3.21 describe the RLS algorithm used to estimate the parameters, $\theta$, of a model given as Equation 3.5.

$$
\begin{gathered}
\tilde{\theta}_{n}=\tilde{\theta}_{n-1}+L(n)\left(y(n)-\phi(n) \tilde{\theta}_{n-1}\right) \\
L(n)=\frac{P(n-1) \phi^{T}(n)}{\lambda+\phi^{T}(n) P(n-1) \phi(n)} \\
P(n)=\frac{P(n-1)-L(n) \phi(n) P(n-1)}{\lambda}
\end{gathered}
$$

where $x(n)$ and $y(n)$ are vectors of length $N$ containing the input and output data respectively, $L(n)$ is the gain vector, $P(n)$ is the covariance matrix of the estimate and $\lambda$ is the forgetting factor.

The RLS algorithm uses changes in the error to track and update the model parameters. The parameters that minimize the prediction error $e(n)$ such that

$$
\begin{aligned}
& e(n)=y(n)-\tilde{y}(n) \\
& \tilde{y}(n)=\phi(n) \tilde{\theta}(n-1) \\
& \left.e(n)=y(n)-\phi(n) \tilde{\theta}_{(} n-1\right)
\end{aligned}
$$


where $y(n)$ and $\tilde{y}(n)$ are the actual and estimated output respectively, $n$ is the time step, and $\left.\tilde{\theta}_{(} n-1\right)$ is the vector of the parameters using $(n-1)$ samples.

The forgetting factor $\lambda$ allows the RLS algorithm to track changing parameters by discounting old data. $\lambda$ can be chosen such that

$$
0.95<\lambda<1
$$

If $\lambda$ is close to 0.95 , RLS algorithm will be able to track parameter changes quickly. If close to 1 , estimates change slowly. The covariance matrix $P(n)$ is defined as $p I$ where $I$ is an identity matrix with rows and columns equal to the number of parameters to be estimated. The term $p \in \Re$ is chosen based on how well the parameters are known. The value $p$ ranges from 0 to an arbitrarily large value. A p value of 0 is chosen when the parameters are well known otherwise, a large p value is used.

\subsection{Review of Adaptive Digital Pre-distortion Schemes}

Adaptive pre-distorter schemes are intended to linearize the output of PAs. Their performance can be measured in terms of speed, complexity and stability. The speed of the pre-distorter is determined by: 1) the time taken at startup for the parameters to converge to values close to the true values for the unknown DPD model and 2) the time taken for the parameters to reconverge after sudden changes in the PA behavior. Complexity depends on the number of computations that are required in the predistorter scheme. Complexity is also determined by the number of samples processed at every estimation and behavioral change. Stability is a measure of how well the parameters transition to the true values at every estimation. 
One of the earliest adaptive PDs reported by [21] offered precise compensation for the nonlinear distortions and adapted to the changes of the PA. The adaptive LUT-based DPD scheme proposed in [17] showed a reduction in convergence time, reconvergence time and complexity compared to [21]. The cascade of a LUT PD and piecewise pre-equalisers proposed in [40] improved on the the LUT-based Hammerstein PD. This method corrected all types of memory effects and simplified the hardware implementation.

An adaptive pre-distorter with a modified LMS algorithms was presented in [29]. A third and fifth order adaptive pre-distorter scheme reported in [16] used a onedimentional search adaptation algorithm to improve the speed and complexity of PAs. However, these adaptive schemes did not evaluate the performance of PAs with fast load changes, especially changes experienced in a base station.

A learning module PD for adaptive PD implementation [41] evaluated with only two discrete power level changes was reported to have rapid convergence of parameters and the ability to learn from past experiences. The PD was demonstrated to improve the transient error and provide implementation at low cost compared to conventional adaptive schemes.

The authors in $[7,42]$ proposed an adpative scheme to suppress distortions due to power changes, but did not validate the scheme using large dynamic ranges. A poweradaptive DPD approach, which avoided parameter recaliberation and eliminated PA distortion due to large dyanmic changes was presented in [43].

\subsection{Improvement to Adaptive DPD Schemes}

Stochastic systems can experience rapid disturbances and multiple parameter changes. Conventional adaptive control schemes for stochastic systems have large transient 
errors during initial estimation and re-estimation of parameters as they transition to their true values. A multiple model control scheme for a controller operating in multiple environments [44] was shown to improve the transient response of the system.

Adaptive control can use multiple models to manage large transients with rapid convergence $[20,45,46]$. A set of models can be used as opposed to a single model used in conventional schemes. The models can have the same structure with equal number of parameters or different structures with different required parameters. Likewise, the same or different algorithms can be used to estimate the model parameters. The model chosen at any instant must provide the best possible linearization performance.

Multiple models require a switching method between the set of models (bank of models). The hypothesis test switching method presented in [47-49] has improved stability than the heuristic performance index switching method proposed in [50].

In this thesis, the adaptive digital pre-distorter is required to provide acceptable linearization performance for a PA. The PA experiences rapid behavioral changes as a result of the changing excitation signal. The parameters of the DPD changes to compensate for the different distortions. The objective is to linearize the PA with increased speed and reduced complexity.

Multiple model adaptive DPD with a hypothesis test based switching method is proposed and evaluated to offer significant improvements over existing conventional adaptive schemes. 


\section{Chapter 4}

\section{Multiple Model Baseband Adaptive Digital Pre-distortion}

\subsection{Introduction}

This chapter explains the multiple model adaptive digital pre-distortion scheme proposed in this thesis. It describes the data employed in the simulation of the DPD. The modeling and simulation procedure of a static and an adaptive DPD are presented.

The synthesis of the multiple model DPD scheme is presented. It improves and builds on the structure of the static and adaptive DPD. The hypothesis test switching algorithm incorporated in the multiple model scheme is presented. The scheme is evaluated based on its linearization performance, complexity and speed.

\subsection{Data Signals Structure}

The input and output data measured from a PA are required for synthesizing a baseband DPD. Figure 4.1 shows the data collection process for the input and output of a PA. These signals can be single-carrier or multi-carrier signals. In baseband, singleor multi-carrier RF signals can be represented by their in-phase (I) and quadrature 
(Q) components. The carrier frequency component of the signals are neglected for simplicity. This form of signal representation is generally referred to as the complex baseband representation of a modulated signal.

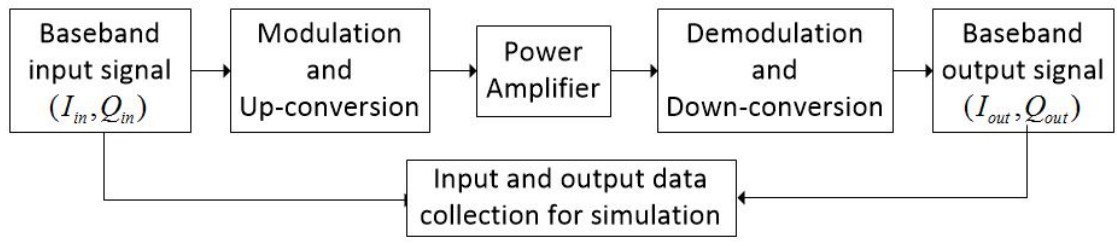

Figure 4.1: Input and output PA data collection

The $I$ and $Q$ components contain all the signal's information and can be used to describe the statistical behavior of the signal. The analysis and synthesis of a DPD is simplified with the use of signals represented in the complex baseband form.

A modulated multi-carrier signal, $x(t)$, in time domain can be defined as

$$
x(t)=\sum_{i} A_{i}(t) \cos \left(w_{i} t+\varphi_{i}(t)\right)
$$

where $A_{i}(t), \varphi_{i}(t)$ and $w_{i}$ are the amplitude, phase and frequency of the $i t h$ carrier that makes up the signal. An equivalent complex baseband notation of a single-carrier $x_{i}(t)$ of the multi-carrier signal $x(t)$ can be defined as

$$
x_{i}(t)=A_{i}(t) \exp \left(j \varphi_{i}(t)\right)
$$

The complex envelope of $x_{i}(t)$ can be represented as

$$
x_{i}(t)=x_{I_{i}}(t)+j x_{Q_{i}}(t)
$$


where,

$$
\begin{aligned}
& A_{i}(t)=\sqrt{x_{I_{i}}(t)^{2}+x_{Q_{i}}(t)^{2}} \\
& \varphi_{i}(t)=\tan ^{-1}\left|x_{Q_{i}}(t), x_{I_{i}}(t)\right|
\end{aligned}
$$

The real $\left(x_{I_{i}}(t)\right)$ and imaginary $\left(x_{Q_{i}}(t)\right)$ values of Equation 4.3 correspond to the in-phase and quadrature components of the signal respectively. The modulated signal $x_{i}(t)$ can be reproduced by multiplying the $I$ and $Q$ components by the cosine and sine of the desired frequency respectively as shown in Equation 4.5.

$$
x_{i}(t)=x_{I_{i}}(t) \cos \left(w_{i} t\right)-x_{Q_{i}}(t) \sin \left(w_{i} t\right)
$$

\subsection{Characterization of the PA}

The measured data signals define or determine the AM/AM and AM/PM characteristics or response of the PA. Thus, these signals can be used to extract the parameters of a model. The number of carriers, PAPR, and the average power of the signal are used in this thesis, to define the statistics of the data. The average power of $N$ samples of $I$ and $Q$ data is defined as

$$
P_{\text {avg }}=10 \log _{10}\left[\frac{1}{N} \sum_{n=1}^{N}|x(n)|^{2}\right][d B m]
$$

where,

$$
|x(n)|=\sqrt{x_{I}(n)^{2}+x_{Q}(n)^{2}}
$$

and $\mathrm{n}$ is the sample index. The PAPR of a signal is defined as

$$
P_{P A P R}=10 \log _{10}\left[\frac{\max \left\{|x(n)|^{2}\right\}}{\frac{1}{N} \sum_{n=1}^{N}|x(n)|^{2}}\right][d B]
$$


where $\max \left\{|x(n)|^{2}\right\}$ is the maximum $\left\{|x(n)|^{2}\right\}$ value computed for all the samples.

\subsection{Time Delay Estimation and Alignment}

The time resolution of the analog-to-digital conversion of the actual PA output signal can result in a time delay between the digitized output and input signal of the PA [51]. It is important to synchronize the time of the measured PA input and output signals and eliminate the time shift between the input and output signals.

The time delay of the PA data signals is estimated and synchronized before the DPD synthesis, using the method proposed in [51]. The time delay estimation/alignment approach presented in [51] applies a lagrange interpolation to the input and output signals. This is done to improve the time resolution of the crosscovariance estimated between the PA input and output signals. The Amplitude and Pre-distorter Simulation Software (AMPS) is utilized to synchronize the PA signals. The software follows the algorithm presented in [51].

\subsection{Scaling of Output Data}

The measured output data is scaled by a factor $(S F)$ that makes the linear gain of

the amplifier equal to 1 and replaces the $\frac{1}{S S G}$ term (SSG is the small signal gain of the PA) after the PA. The scaled output $y_{P \text { Ascaled }}(\mathrm{n})$ can be derived from the time aligned measured output data $y_{\text {timealigned }_{P A}}(\mathrm{n})$ as

$$
y_{P \text { Ascaled }}(\mathrm{n})=y_{\text {timealigned }_{P A}}(\mathrm{n}) \times \mathrm{SF}
$$


where,

$$
S F=\frac{\frac{1}{N} \sum_{n=1}^{N}\left(\mid x_{\text {timealigned } \left._{P A}(\mathrm{n}) \mid\right)}\right.}{\frac{1}{N} \sum_{n=1}^{N}\left(\mid y_{\text {timealigned }_{P A}(\mathrm{n}) \mid}\right)}
$$

and $\mathrm{N}$ is the number samples and $S F=\frac{1}{S S G}$.

\subsection{Data Pre-processing}

The peak average power ratio (PAPR) of the pre-distorted signal (DPD output) is higher than the PAPR of the actual PA input signal. Such phenomenon is referred to as DPD avalanche [52]. However, the PA is characterized with the actual input at its specific PAPR. When the PA is excited by the DPD's output signal with a higher PAPR, the PA is pushed more into saturation. Thus, the DPD in this case will only degrade the achievable linearization performance of the PA.

Higher order polynomial-based models can improve the accuracy of the estimated PA model. However, higher model orders increase the model parameters to be estimated and the numerical instability of the model parameters causing large dispersions in the parameters. Dispersions are the magnitude of the separation between the maximum and minimum parameter values.

The higher peaks and large parameter dispersions can be controlled and reduced by pre-processing the data signals $[38,52-54]$. The pre-processing of a signal $x(n)$ is described as

$$
x_{p r e-p}(\mathrm{n})=\frac{x(n)-\bar{x}}{\sigma}
$$

where $\bar{x}$ and $\sigma$ are the mean and standard deviation of the signal $x(n)$ respectively.

The notations, $x_{P A}(\mathrm{n})$ and $y_{P A}(\mathrm{n})$, that appear in the remaining sections of this thesis represent the time-aligned, scaled and pre-processed input signal and output 
signal respectively. It should be noted that the input signal is not scaled and the pre-processing step operates on the time aligned input signal.

\subsection{Static Digital Pre-distortion Synthesis}

The synthesis structure is the procedure for realising DPD via simulation. Figure 4.2 illustrates the flowchart of a static DPD implementation via simulation. First, the parameters of the PA and DPD model are estimated by an offline one-time model identification algorithm that uses the input $\mathrm{x}_{P A}(\mathrm{n})$, and output $\mathrm{y}_{P A}(\mathrm{n})$ PA data. Second, the PA input is passed through a cascade of the PA and DPD model (DPD+PA).

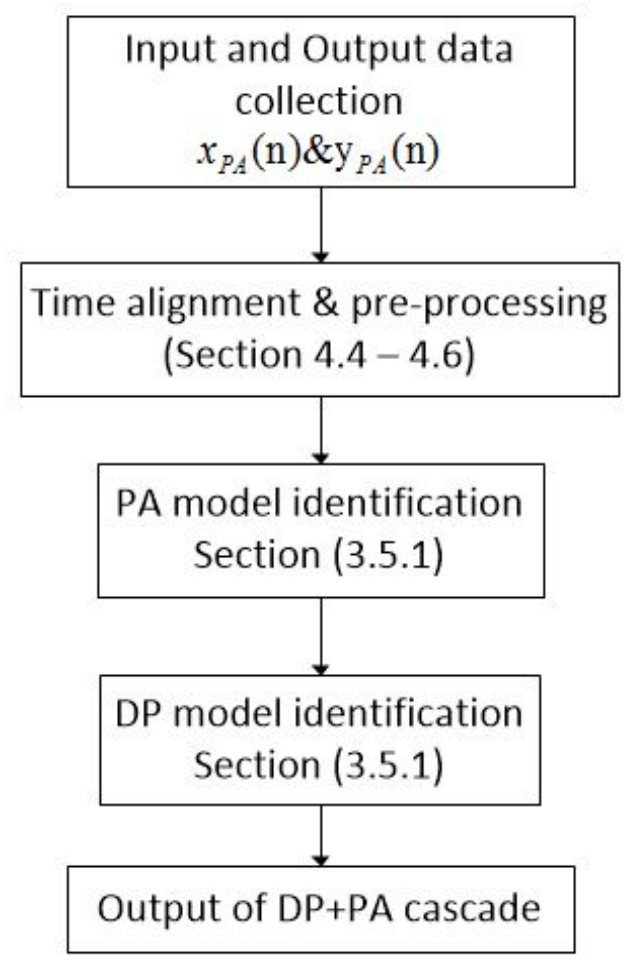

Figure 4.2: Flowchart for the static DPD implementation

The procedure below describes the synthesis of a static DPD: 
- Let us assume that the characteristics of the PA and DPD can be described by a polynomial model (MP) of the form

$$
\begin{gathered}
y_{P A}(n)=\sum_{k=1}^{K_{P A}} \sum_{m=0}^{M_{P A}-1} a_{k m_{P A}} x_{P A}(n-m)\left|x_{P A}(n-m)\right|^{k-1} \\
y_{D P}(n)=\sum_{k=1}^{K_{D P}} \sum_{m=0}^{M_{D P}-1} a_{k m_{D P}} x_{D P}(n-m)\left|x_{D P}(n-m)\right|^{k-1}
\end{gathered}
$$

where $\mathrm{x}_{P A}(\mathrm{n}), y_{P A}(\mathrm{n}), \mathrm{a}_{k m_{P A}}, M_{P A}$, and $K_{P A}$ are associated with the PA model while $\mathrm{x}_{D P}(\mathrm{n}), y_{D P}(\mathrm{n}), \mathrm{a}_{k m_{D P}}, M_{D P}$, and $K_{D P}$ are associated with the DPD model. The signals $x(n)$ and $y(n)$ are the pre-processed input and output data respectively, $a_{k m}$ are the complex-valued model parameters, $K$ is the highest nonlinear order and $M$ is the memory length of the model to be estimated.

The terms $K$ and $M$ specify the model configuration. The model structure is the form of the mathematical representation of the PA and DPD model. It should be noted that the PA model structure and configuration can be chosen differently from that of the DPD. Table 4.1 shows the possible combinations of a PA and DPD model structure and configuration.

Table 4.1: Possible PA and DPD model structure and configuration choice

\begin{tabular}{ccc}
\hline \hline Options & Model Structure & Model Configuration \\
\hline \hline Option 1 & Identical & Identical \\
Option 2 & Identical & Different \\
Option 3 & Different & Identical \\
Option 4 & Different & Different \\
\hline
\end{tabular}

The optimum option uses the smallest configuration possible and the simplest model structure that yields acceptable linearity with high speed and less complexity. 
- The LS algorithm described in Section 3.5.1 can be used for estimating the parameters of the PA model. The parameters of the PA, $\mathrm{a}_{k m_{P A}}$, and the DPD, $\mathrm{a}_{k m_{D P}}$, can be estimated as

$$
\begin{gathered}
\tilde{\mathrm{a}}_{k m_{P A}}=\phi_{P A}{ }^{+}(n) y_{P A}(n) \\
\tilde{\mathrm{a}}_{k m_{D P}}=\phi_{D P}{ }^{+}(n) y_{D P}(n)
\end{gathered}
$$

where $\phi_{P A}{ }^{+}(n)$ and $\phi_{D P}{ }^{+}(n)$ are the pseudoinverse of the regression matrix $\phi_{D P}(n)$ and $\phi_{D P}(n)$ respectively. It should be noted that the PA's input signal $x_{P A}(\mathrm{n})$ becomes the DPD's input signal $x_{D P}(\mathrm{n})$ while the PA's output signal $\mathrm{y}_{P A}(\mathrm{n})$ becomes the DPD's input signal $\mathrm{y}_{D P}(\mathrm{n})$ for modeling the DPD.

- A cascade of the PA and DPD is desired to produce the linearized output, $y_{\text {linearizedPA }}(\mathrm{n})$, similar to the actual PA input. The actual PA input data, $\mathrm{x}_{P A}(\mathrm{n})$, is passed through the DPD to yield $\mathrm{y}_{D P}(\mathrm{n})$. Then, $\mathrm{y}_{D P}(\mathrm{n})$ serves as the new PA input $\mathrm{x}_{\text {newPA }}(\mathrm{n})$. Equations 4.16 and 4.17 describe the derivation of the linearized PA output.

$$
\begin{aligned}
& \mathrm{y}_{D P}(\mathrm{n})=\phi_{P A}{ }^{T} \tilde{\mathrm{a}}_{k m_{D P}}=\mathrm{x}_{n e w P A}(\mathrm{n}) \\
& y_{\text {linearizedPA }}(\mathrm{n})=\phi_{n e w P A}{ }^{T}(\mathrm{n}) \tilde{\mathrm{a}}_{k m_{P A}}
\end{aligned}
$$

For a perfectly linearized PA,

$$
y_{\text {linearizedPA }}(\mathrm{n}) \simeq x_{P A}(\mathrm{n})
$$


The DPD can only be modeled offline because its estimation algorithm is nonadaptive. When a change in the PA behavior results in a poor linearization performance, $N$ samples of the PA have to be re-collected for the DPD to be re-trained offline for the new operating condition.

\subsection{Adaptive Digital Pre-distortion Synthesis}

The procedure for the synthesis of an adaptive DPD is similar to the static DPD except for the type of estimation algorithm. The identification algorithm can adjust the DPD parameters and the DPD can continually compensate for the varying distortions of the PA. Figure 4.3 illustrates the flowchart of an adaptive DPD implementation. The characteristics of the PA and DPD can be described by a polynomial model of

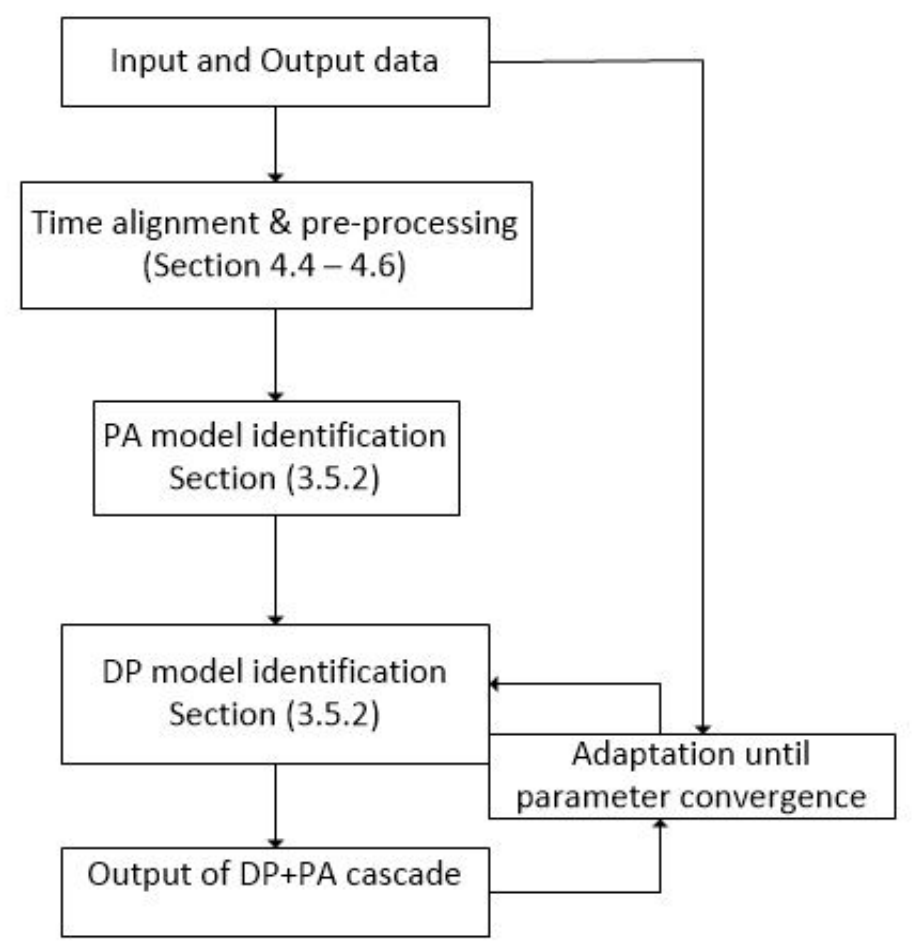

Figure 4.3: Flowchart of an adaptive DPD implementation 
the form

$$
\begin{aligned}
& y_{P A}(n)=\sum_{k=1}^{K_{P A}} \sum_{m=0}^{M_{P A}-1} a_{k m_{P A}} x_{P A}(n-m)\left|x_{P A}(n-m)\right|^{k-1} \\
& y_{D P}(n)=\sum_{k=1}^{K_{D P}} \sum_{m=0}^{M_{D P}-1} a_{k m_{D P}} x_{D P}(n-m)\left|x_{D P}(n-m)\right|^{k-1}
\end{aligned}
$$

where $\mathrm{x}_{P A}(\mathrm{n}), y_{P A}(\mathrm{n}), \mathrm{a}_{k m_{P A}}, M_{P A}$, and $K_{P A}$ are associated with the PA model while $\mathrm{x}_{D P}(\mathrm{n}), y_{D P}(\mathrm{n}), \mathrm{a}_{k m_{D P}}, M_{D P}$, and $K_{D P}$ are associated with the DPD model. The terms $x(n)$ and $y(n)$ are the pre-processed input and output data respectively, $a_{k m}$ are the complex-valued model parameters, $K$ is the highest nonlinear order and $M$ is the memory length of the model to be estimated.

The PA parameters, $a_{k m_{P A}}$, and DPD parameters, $a_{k m_{D P}}$ can be estimated using

$$
\begin{gathered}
\tilde{\mathrm{a}}_{k m_{P A}}(n+1)=\tilde{\mathrm{a}}_{k m_{P A}}(n)+\left(\mu * \phi_{P A}^{T}(n) * e_{P A}(n)\right) \\
\tilde{\mathrm{a}}_{k m_{D P}}(n+1)=\tilde{\mathrm{a}}_{k m_{D P}}(n)+\left(\mu * \phi_{D P}^{T}(n) * e_{D P}(n)\right)
\end{gathered}
$$

The estimation error $e(n)$ between the actual PA input $\mathrm{x}_{P A}(\mathrm{n})$ and the estimated output $\tilde{y}_{D P+P A}(\mathrm{n})$ of the cascade of the DPD and PA model, controls the algorithm. The error $e(n)$ is written as

$$
\mathrm{e}(\mathrm{n})=\mathrm{x}_{P A}(\mathrm{n})-\tilde{y}_{D P+P A}(\mathrm{n})
$$

New $x_{P A}(n)$ and $y_{P A}(n)$ samples can cause large errors in the estimation of the DPD parameters. The adaptive schemes described above are prone to large transient errors and the linearization performance is poor. Large changes in parameters degrade the linearization performance of the DPD. This requires larger sample sizes to be processed before parameters converge to optimal values to achieve the linearity performance specified by standards. 


\subsection{Multiple Model Digital Pre-distortion}

The multiple model DPD scheme is intended to reduce the time taken for the DPD to optimize its parameters for the present operating condition (start-up and during system changes). Figure 4.4 shows a block diagram of the multiple model DPD scheme. The scheme uses a set of DPD models $\left(D P M_{i}\right)$ each with its own set of parameters. Each $D P M_{i}$ is related to a specific operating condition of the PA. The parameters are grouped, stored and addressed in memory based on their power level and bandwidth as shown in Figure 4.5.

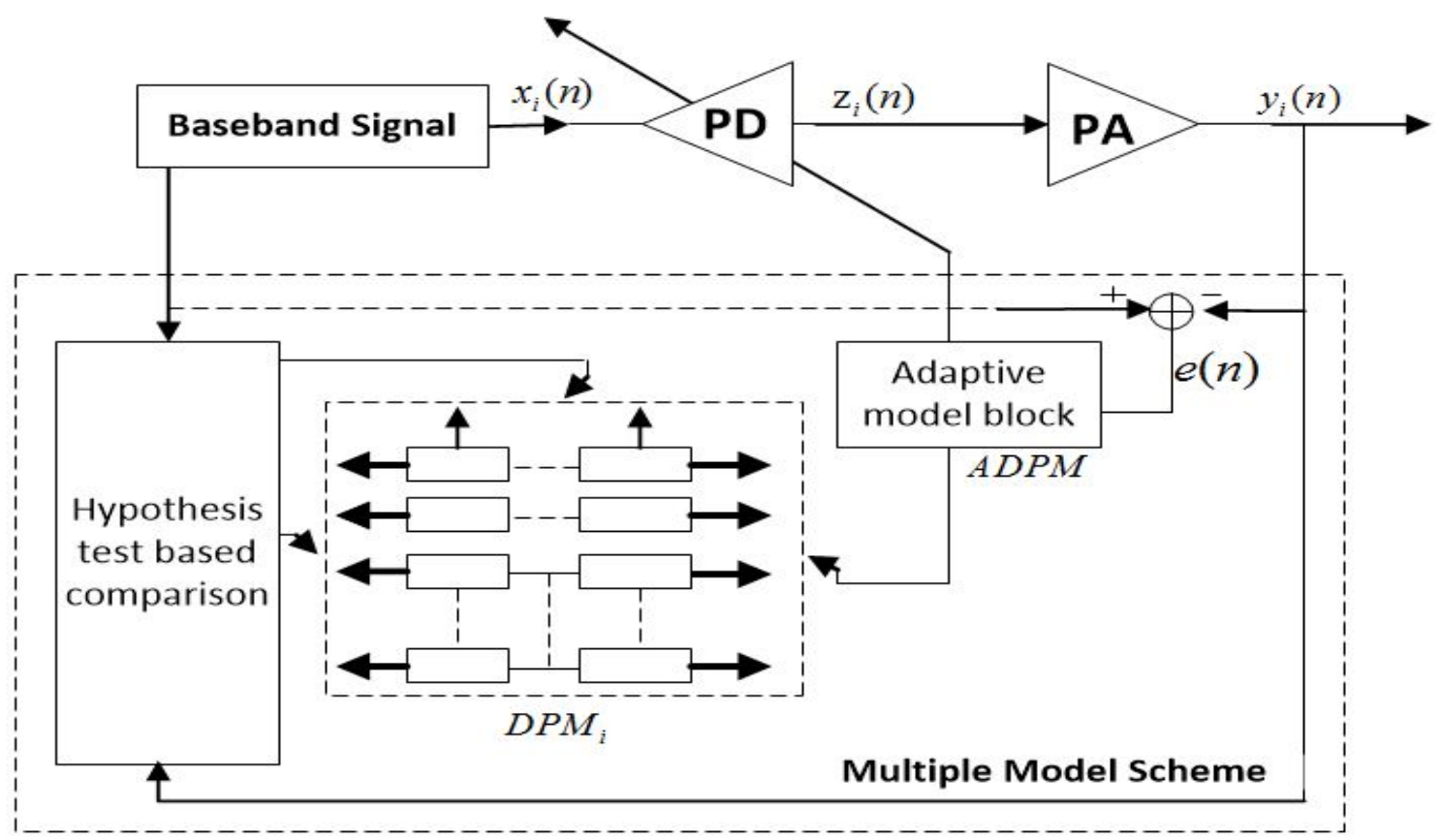

Figure 4.4: Block diagram of the multiple model scheme

The DPD models are switched based on the operating condition of the PA and related to the input signal statistics at any given instant. A hypothesis test based switching algorithm is employed to determine model switching. When a switch is initiated by the algorithm, a certain set of $D P M_{i}$ model parameters are selected. In response to the selection of a $D P M_{i}$ model after the switching decision, the adaptive 
model block is re-initialized using the initial parameters of the $D P M_{i}$ model selected. However, when a switch is not initiated, the input signal and operating conditions of the PA remained constant and the scheme uses previously estimated parameters for the DPD. The resulting parameters from the adaptive model at a particular condition

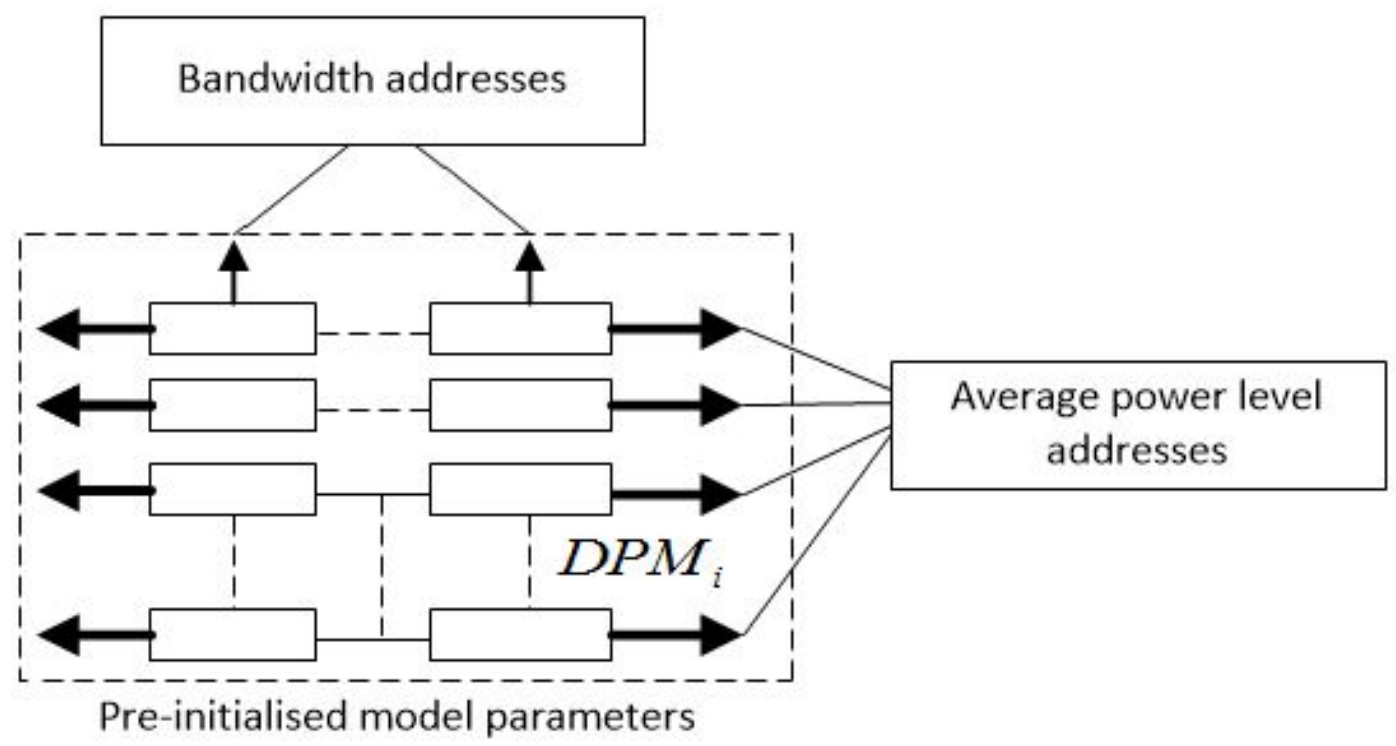

Figure 4.5: Multiple model DPD parameters addressing

can be stored after every adaptation and used to reset the initial parameters of $D P M_{i}$ model. The scheme can be described in the following steps:

a Offline parameterization for model placement

b Multiple models are initialized with parameters extracted from Step 1.

c The switching algorithm implementation

d Adaptation of selected initial parameters to fit current data 


\subsubsection{Offline Parameterization for Model Placement}

The model parameters for each DPD model $\left(D P M_{i}\right)$ are initialized at specific values to minimize transient degradation [41]. The initial values of the model parameters are determined before the PA startup. Input data, with different statistics, are used to excite the PA and their corresponding output data are retrieved. A specific input data and its corresponding output data are referred to as a dataset. The signals used for initialization are representative of the actual signals to be experienced by the PA in its real-time operation.

Initial parameters of each model are identified using the procedure similar to the static DPD synthesis described in Section 4.7. A DPD model, $\left(D P M_{i}\right)$, can be defined by a memory nonlinear polynomial model structure of the form

$$
y_{i}(n)=\sum_{k=1}^{K_{i}} \sum_{m=0}^{M_{i}-1} a_{k m_{i}} x_{i}(n-m)\left|x_{i}(n-m)\right|^{k-1}
$$

where $i$ represents the power level index of a batch of input signals that categorize an $i$ th operating condition (See Figure 4.6), $x_{i}(n)$ and $y_{i}(n)$ are the input and output DPD data respectively with unique characteristics, $a_{i_{k m}}$ are the model parameters, $K_{i}$ is the highest nonlinear order and $M_{i}$ is the memory length. $x_{i}(n), y_{i}(n), a_{i_{k m}}, M_{i}$, and $K_{i}$ are associated with a DPD model $\left(D P M_{i}\right)$. The terms $K_{i}$ and $M_{i}$ are real integer values that determine the configuration of the model structure. The configuration and structure of each $D P M_{i}$ can be identical or vary across all models. The number of samples used to estimate the complex-valued parameters, $a_{i_{k m}}$ (Equation 4.25), of each $\left(D P M_{i}\right)$, at the ith operating condition must be adequate enough to minimize 


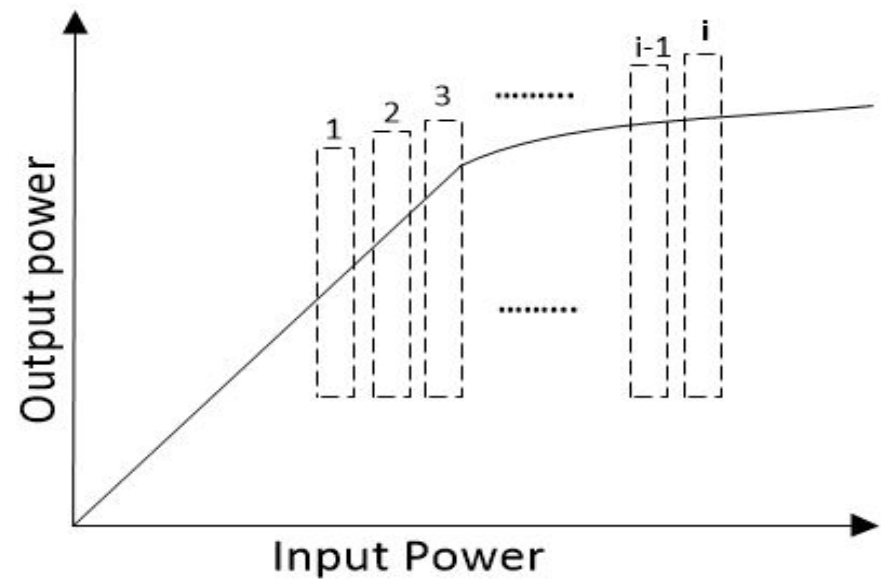

Figure 4.6: Characteristic plot showing the operation of the PA over $i$ distinct operating conditions

the cost function $E\left[e^{2}(n)\right]$ defined by Equation 4.26 .

$$
a_{i_{k m}}^{T}=\left[\begin{array}{llllllllll}
a_{i_{10}} & a_{i_{11}} & a_{i_{1 M}} & a_{i_{20}} & a_{i_{21}} & a_{i_{2 M}} & \cdots & a_{i_{K 1}} & a_{i_{K 2}} & a_{i_{K M}}
\end{array}\right]
$$

where $(.)^{T}$ is the transpose operator.

$$
E\left[e_{i}^{2}(n)\right]=\sum_{n}\left(y_{i}(n)-\tilde{y}_{i}(n)\right)^{2}
$$

where $y_{i}(n)$ and $\tilde{y}_{i}(n)$ are the actual output and estimated output respectively associated with the $i t h$ operating condition modeled by $D P M_{i}$, and $\tilde{y}_{i}(n)$ is given as

$$
\tilde{y}_{i}(n)=\phi_{i}(n) a_{k m_{i}}
$$

and $\phi_{i}^{T}(n)$ is the regression matrix formulated from the input signal $x_{i}(n)$ associated 
with the model $\left(D P M_{i}\right)$.

$$
\phi_{i}(n)=\left[\begin{array}{ccccccc}
x_{i 10} & \cdots & x_{i 1(M-1)} & \cdots & x_{i 11}\left|x_{i 11}\right|^{K-1} & \cdots & x_{i 1(M-1)}\left|x_{i 1(M-1)}\right|^{K-1} \\
x_{i 20} & \cdots & x_{i 2(M-1)} & \cdots & x_{i 21}\left|x_{i 21}\right|^{K-1} & \cdots & x_{i 2(M-1)}\left|x_{i 2(M-1)}\right|^{K-1} \\
\vdots & \vdots & \vdots & \cdots & \vdots & \vdots & \vdots \\
x_{i N 0} & \cdots & x_{i N(M-1)} & \cdots & x_{i N 1}\left|x_{i N 1}\right|^{K-1} & \cdots & x_{i N(M-1)}\left|x_{i N(M-1)}\right|^{K-1}
\end{array}\right]
$$

The pre-initialised parameters are stored in the memory of the multiple model scheme. Each set of parameters are addressed based on the statistics of the input signal that define the PA behavior and operating condition. Figure 4.7 and 4.8 illustrate the parameter initialization process.

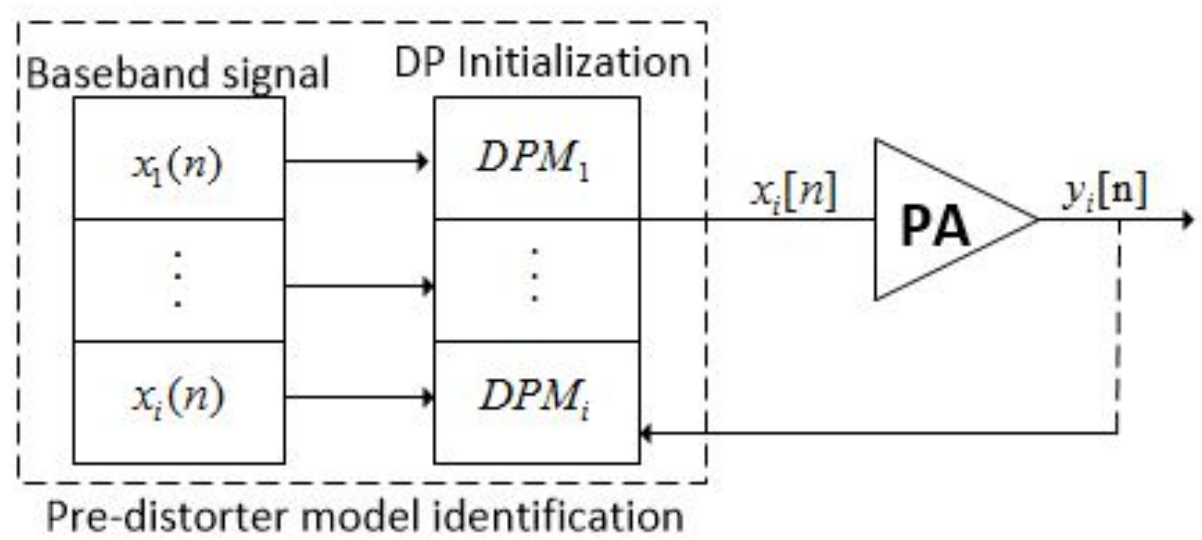

Figure 4.7: DPD scheme parameter initialization

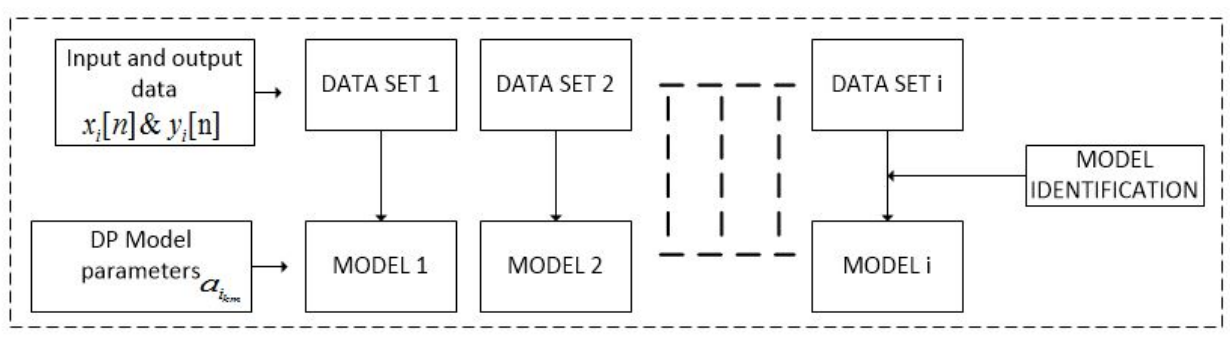

Figure 4.8: Model extraction 


\subsubsection{Hypothesis Test Switching Algorithm}

The purpose of multiple model control is to reduce the size of the transient error and adapt faster to the changes. Therefore, the decision of which model to pick is made quickly and the selected model should yield the best results when compared to other models. The purpose is to achieve fast switching by improving convergence to the new parameter values and accuracy of selection. The $D P M_{i}$ model selected at every time must give the minimum error $E\left[e_{i}^{2}(n)\right]$ between the original input and linearized output of the PA.

The decision to switch is based on an assertion that the characteristics of the input signal to the PA, has changed. It is beneficial to make use of inferential statistics which allows measurement of the properties of the data to detect the changes in the PA. The hypothesis test based switching algorithm [48] is used to determine changes to the input signal of the PA. The algorithm ensures data consistency for the case study presented in this thesis (input power level and bandwidth changes).

The switching algorithm is based on two hypothesis: 1) the null hypothesis $H_{o}$ and, 2) the alternative hypothesis $H_{a} . H_{o}$ represents a constant PA behavior and assumes the input data to the PA is not changing. The hypothesis to be proven is the alternative hypothesis $H_{a}$. The purpose of the algorithm is strictly to: Reject the null hypothesis and accept the alternative if and only if the results of the hypothesis test are statistically significant.

The hypothesis algorithm can be explained in the following steps:

a State the null hypothesis. In this thesis, the null hypothesis is that the statistics of the input signal is unchanging.

b Compute the test statistic for statistical significance

c Decision. Accept or reject null hypothesis. 
The hypothesis is set up as a two-tailed alternative. In the two-tailed test, the result of the test must be supported by evidence in one of the two directions. Results in either of the two directions can lead to the rejection of the null nypothesis. When the alternative hypothesis is accepted, the model with the minimum error is selected.

\section{Test Statistic}

The t-test observes the changes in the signal statistics (average power and bandwidth). The statistics of the pre-processed data signal approximately follows a Gaussian distribution making the t-test appropriate to monitor statistical changes. The change in statistics of the signal is observed in the t-test result $t_{o}(k)$ which is compared to a user-defined threshold value $T_{h}$. The comparison guides the decision of either accepting or rejecting the null hypothesis. The value of $T_{h}$ corresponds to a significance level, $\alpha$, using the statistical t-table.

If $\quad \mathrm{N} \quad$ samples $\quad\left(x_{a}(1), x_{a}(2), \ldots, x_{a}(N)\right) \quad$ containing $\left(\left(I_{a}(1), Q_{a}(1)\right),\left(I_{a}(2), Q_{a}(2)\right), \ldots,\left(I_{a}(N), Q_{a}(N)\right)\right)$ selected from the current batch $a$ of input data are compared with another $\mathrm{N}$ samples $\left(x_{b}(1), x_{b}(2), \ldots, x_{b}(N)\right)$ containing $\left(\left(I_{b}(1), Q_{b}(1)\right),\left(I_{b}(2), Q_{b}(2)\right), \ldots,\left(I_{b}(N), Q_{b}(N)\right)\right)$ selected from the next batch $b$ of PA input data, the test statistic (t-test) can be defined as

$$
t_{o}(k)=\frac{\frac{1}{N}\left[\sum_{n=1}^{N}\left(x_{a}(n)-x_{b}(n)\right)\right]}{S(k) / \sqrt{N}}
$$

where $k$ represents the decision index, and $S(k)$ is the running standard deviation. $x_{a}(n)$ and $x_{b}(n)$ are absolute values of the signal sequence. The running standard 
deviation, $S(k)$ is defined as

$$
S(k)=\sqrt{\sum_{n=1}^{N} \frac{\left(x_{a}(n)-x_{b}(n)\right)}{N-1}}
$$

The decision to switch indexed at $k$ is accepted if and only if:

- $t_{o}(k) \leq-T_{h}$ or

- $t_{o}(k) \geq+T_{h}$

For values of $t_{o}(k)$ that lie between $-T_{h}$ and $+T_{h}$, there is no reason to switch and the scheme simply maintains the previous model parameters. In the event of a change in PA characteristics caused by the varying dataset, a switch is permitted to another model that will yield the least error to ensure model accuracy and maintain the linearization performance.

\section{Switching Accuracy and Speed}

The sample size, $\mathrm{N}$, used in the test statistic computation determines the accuracy of the hypothesis test. A large sample size increases the accuracy but at the cost of reduced algorithm speed. A smaller sample size reduces the accuracy of the PA

and increases its speed. The sample size and threshold value are selected to ensure switches happen when input statistics changes and false switches are not accepted.

The decision to switch is false when the statistics of the input signal and the PA's behavior remain unchanged but the test algorithm initiates a switch. In this thesis, the minimum number of samples that can achieve accuracy and still maintain speed is determined by a trial and error method. The hypothesis test is a solution to deciding online the best model to use at a given point in time. 


\subsubsection{Selected Model Adaptation}

The DPD model selected at every instant generates the minimum error between the measured PA input and the linearized PA output. This selection ensures the linearization performance is maintained. When the switching algorithm is active, a set of parameters $\mathrm{a}_{i_{k m}}$, of a model $\left(D P M_{i}\right)$ associated with an ith operating point, are selected. The selected parameters are used to re-initialize the parameters of the adaptation model. The adaptive algorithms of Section 3.5.2 initialized using these parameters are used for adaptation for parameters to re-converge to give the best possible linearization performance.

Initialization at parameters estimated in advance helps when little is known about the PA's operating condition (at start-up and behavior changes). When the power level of the input signal lies betweem pre-determined ith levels, the statistics of the data can vary slightly or significantly from the PA data used to estimate the parameters for an ith level. However, adaptation from the initialized parameters associated with any of the adjacent $i t h$ levels can help maintain zero control error. The estimated parameters derived from adaptation are fed into the pre-distorter block continually to update the DPD parameters. Figure 4.9 illustrates the flowchart for the multiple model scheme.

The parameters estimated from adaptation for a particular operating condition can be used to reset the parameters of the fixed $D P M_{i}$ model gotten from the offline learning. This is the online learning. When an operating condition is experienced again, the parameters selected for adaptation from the set of $D P M_{i}$ are the ones learned from adaptation and not the offline-learned parameters. 


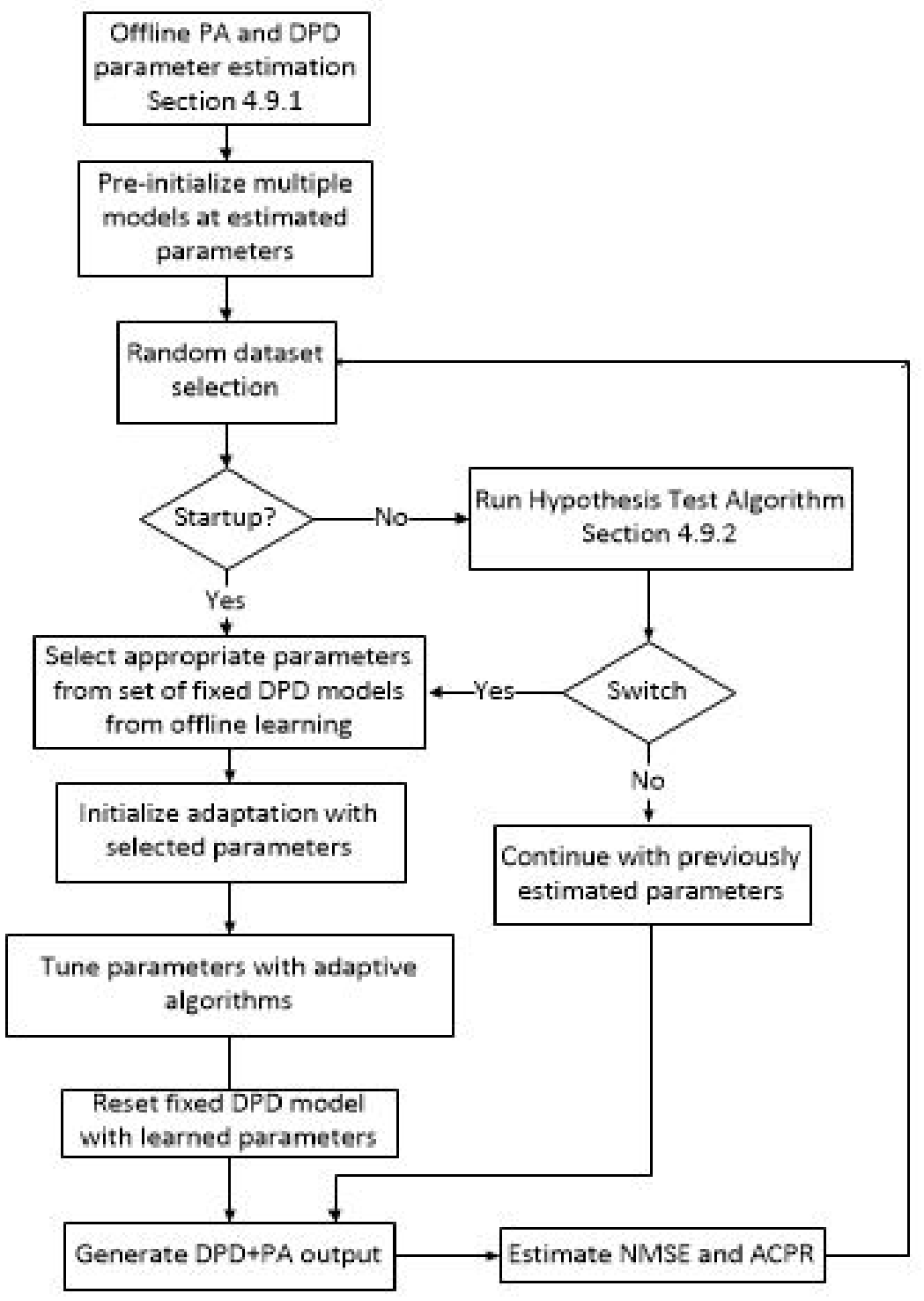

Figure 4.9: Flowchart of the multiple model scheme 


\subsection{Multiple Model Configuration}

The multiple model digital pre-distorter is more flexible than the single model adaptive schemes because each DPD model $D P M_{i}$ can be set to have different model structures and configurations. A uniform configuration for DPD models with the same structure means that the nonlinear order $K_{i}$ and memory depth $M_{i}$ for each model are the same. Thus, each model estimate generates an equal number of parameters.

The total number of parameters to be estimated can be reduced by using different model configurations for different $i t h$ power levels. This is based on the idea that fewer number of parameters are adequate to model a PA operating at a low power than a PA at a higher power. Thus, the value of the $K_{i}$ and $M_{i}$ set for every power level is not equal. Moreover, the total number of parameters to be stored in memory and the overall complexity of the scheme can be significantly reduced during low power conditions. For all configuration scenerios considered in this thesis, it is assumed that identical model structures and estimation algorithms are used for all DPD models.

1 Scenario I - Uniform configuration for ith DPD models

- For a multiple model DPD containing $D P M_{1}$ to $D P M_{i}$ models, the choice of $K_{i}$ and $M_{i}$ for each $D P M_{i}$ are the same. The number of parameters estimated in each model are equal. i.e.

$$
K_{1} M_{1}=K_{2} M_{2}=\cdots=K_{n} M_{n}
$$

where $\mathrm{n}$ is the maximum model number.

2 Scenario II - Non-uniform configuration for ith DPD models 
- For a multiple model DPD containing $D P M_{1}$ to $D P M_{i}$ models, the choice of $K_{i}$ and $M_{i}$ for each $D P M_{i}$ are not the sa,e. The number of parameters estimated in each model $D P M_{i}$ are different from one another. The terms $K_{i}$ and $M_{i}$ can be chosen as

$$
K_{1} M_{1} \leq K_{2} M_{2} \leq \cdots \leq K_{n} M_{n}
$$

such that the sum of all parameters $\sum_{i} K_{i} M_{i}<n \times K_{n} M_{n}$

\subsection{Scheme Evaluation}

The DPD scheme proposed in this thesis is evaluated based on its linearization performance, the time taken to arrive at acceptable linearization performance (speed) and the computational complexity.

\subsubsection{Linearization Performance}

An effective linearization scheme is demonstrated by a significant reduction or complete removal of the in-band distortions and the power interfering in the adjacent channels otherwise known as the out-of-band emissions seen as spectral regrowth. The linearization performance of the proposed DPD scheme is evaluated using the following metrics: 1) the ACPR (frequency domain linearization performance) and 2) the NMSE (time donamin linearization performance).

Digital signal standards such as the 3rd Generation Partnership Project (3GPP) standard specifies the maximum acceptable ACPR of $-45 \mathrm{dBc}$ at a $5 \mathrm{MHz}$ offset, $-50 \mathrm{dBc}$ at a $10 \mathrm{MHz}$ offset and the maximum acceptable NMSE value of $-35 \mathrm{~dB}$ for WCDMA signal formats. [10]. The linearization performance is accepable when the measured 
ACPR and NMSE are less than the maximum requirements.

The ACPR measures the power in the adjacent channels of the linearized output as a ratio of the power in its main channel. The frequency domain performance can also be visualized from the power spectrum density (PSD) plot. The plot shows how much spectrum regrowth in the linearized output has been suppressed compared to the original measured output. The PSD in this thesis is estimated using the Welchs method. The ACPR is calculated as

$$
A C P R=\max _{m=1,2 \ldots} \frac{\int_{(\text {adj }) m}\left|Y_{\text {linearized }}(f)\right|^{2} d f}{\int_{c h}\left|Y_{\text {linearized }}(f)\right|^{2} d f}
$$

where $Y_{\text {linearized }}(f)$ is the Fourier transform of the DPD+PA output, $\int_{(a d j) m} Y_{\text {linearized }}(f) d f$ measures the power in the adjacent channel at $m$ offsets, and $\int_{(c h) m} Y_{\text {linearized }}(f) d f$ measures the power in the main channel.

The NMSE compares the input and linearized output in the time domain. The NMSE evaluates the compensation of in-band distortions (in-band performance) and the accuracy of the time domain signal after linearization. The NMSE is calculated as

$$
N M S E(d B)=10 \log _{10}\left[\frac{\sum_{n}\left|x(n)-y_{\text {linearized }}(n)\right|^{2}}{\sum_{n}|x(n)|^{2}}\right]
$$

Equations 4.33 and 4.34 are similar to the ones previously presented in Section 2.3.2 and 2.3.2 respectively. The previous equations are applied to the measured PA input and output as opposed to the PA input and linearized PA output (DPD+PA) used here. The performance of an adaptive DPD scheme is also dependent on the accuracy of the model estimation. However, the accuracy of the estimated model is dependent on the model estimation algorithm. 


\subsubsection{Complexity and Speed}

Complexity is determined by the amount of mathematical operations that are involved in achieving the DPD $[11,23]$. It is evaluated in terms of the number of additions, subtractions, divisions, and multiplications required in a computation. In real hardware, complex operations are converted into real operations and memory size has to be considered. Convergence speed is the time taken for adjusting estimated model parameters until linearization performance is acceptable.

The speed and complexity of a DPD scheme are interpreted from the number of samples required to be processed per unit time and the number of parameters to be estimated. Larger valued parameters require higher resolution digital circuits to process and store them. An increased number of samples translates to larger matrices computations which significantly increases complexity.

For accurate model estimation, large sample sizes are required to prevent illconditioned matrices in the estimation. Smaller sample sizes are not fully statistically representative of the real data and can degrade the linearization performance of the DPD [55]. A trade-off between the sample size required and degree of estimation accuracy has to be considered. The smallest sample size possible that can provide an acceptable degree of accuracy can be selected.

A matrix multiplication $A_{I \times J} B_{J \times L}$ will require $O^{\otimes} A_{I \times J} B_{J \times L}$ complex multiplications, and $O^{\oplus} A_{I \times J} B_{J \times L}$ additions such that

$$
\begin{gathered}
O^{\otimes}{ }_{I \times J * B_{J \times L}}=J(I \times L) \\
O^{\oplus}{ }_{A_{I \times J} B_{J \times L}}=I \times L(J-1)
\end{gathered}
$$

where $A$ has $I$ rows and $J$ columns, $B$ has $J$ rows and $L$ columns, and the elements of 
$A$ and $B$ are complex valued. The computation of a matrix inversion $C_{I \times I^{-1}}$, requires $O^{\otimes} C_{I \times I}{ }^{-1}$ multiplications such that

$$
O^{\otimes} C_{I \times I}{ }^{-1}=I^{3}
$$

where $C$ has $I$ rows and columns and the elements of $C$ are complex valued. A complex multiplication is equivalent to four real multiplications [56].

The LS based estimation written as

$$
\tilde{\theta}_{K \times 1}=\left(\phi_{K \times N}^{H} \phi_{N \times K}\right)^{-1} \phi_{K \times N}^{H} y(n)_{N \times 1}
$$

where $\tilde{\theta}$ represents the parameter vector with $K$ elements, $(.)^{H}$ represents the hermitian or complex conjugate transpose, $\phi(n)$ represents the regression matrix having $N$ rows and $K$ columns, and $y(n)$ represents the output data vector of length $N$, requires $O^{\otimes}{ }_{L S}$ multiplications and $O^{\oplus}{ }_{L S}$ additions where

$$
\begin{gathered}
O^{\otimes}{ }_{L S}=N \times K^{2} \times 2+N \times K+K^{3} \\
O^{\oplus}{ }_{L S}=(N-1) \times K^{2}+N \times(K-1) \times K+(N-1) \times K
\end{gathered}
$$

Computation of the complex-valued parameters per sample using the LMS algorithm $O^{\otimes}{ }_{L M S}$ given in Equation 3.15 requires $K$ complex multiplications while the computation using the RLS estimator $O_{R L S}^{\otimes}$ given in Equation 3.19 requires $K^{2}$ complex multiplications. Thus, the computation complexity per sample for the RLS is higher than the LMS algorithm. The computation of model parameters with an identification algorithm using $<N$ samples will be faster than using $\geq N$ samples. Likewise, the complexity of the former will be reduced compared to the latter. 


\section{Chapter 5}

\section{Simulation Results}

In this chapter, the results of the simulations performed in MATLAB are presented. Simulations are performed to demonstrate the performance of existing static and adaptive DPD schemes presented by other authors. Simulations are also used to implement and evaluate the proposed multiple model adaptive DPD scheme. The effectiveness of the proposed scheme (in terms of linearization performance, complexity and speed) is assessed using real experimental data measured from a PA under test. The simulations follow the DPD synthesis, data pre-processing, time alignment, and hypothesis test switching algorithms outlined in Chapter 4.

\subsection{Testbed Equipment Platform Setup}

An experimental platform was utilized to generate the data of a real PA. This experimental platform consists of a personal computer $(\mathrm{PC})$, a vector signal generator (VSG), a driver amplifier, the device under test (DUT) i.e the PA, an attenuating load, and a spectral analyser (SA). Figure 5.1 shows the schematic diagram of the experimental platform. The function of the components of the experimental platform are 


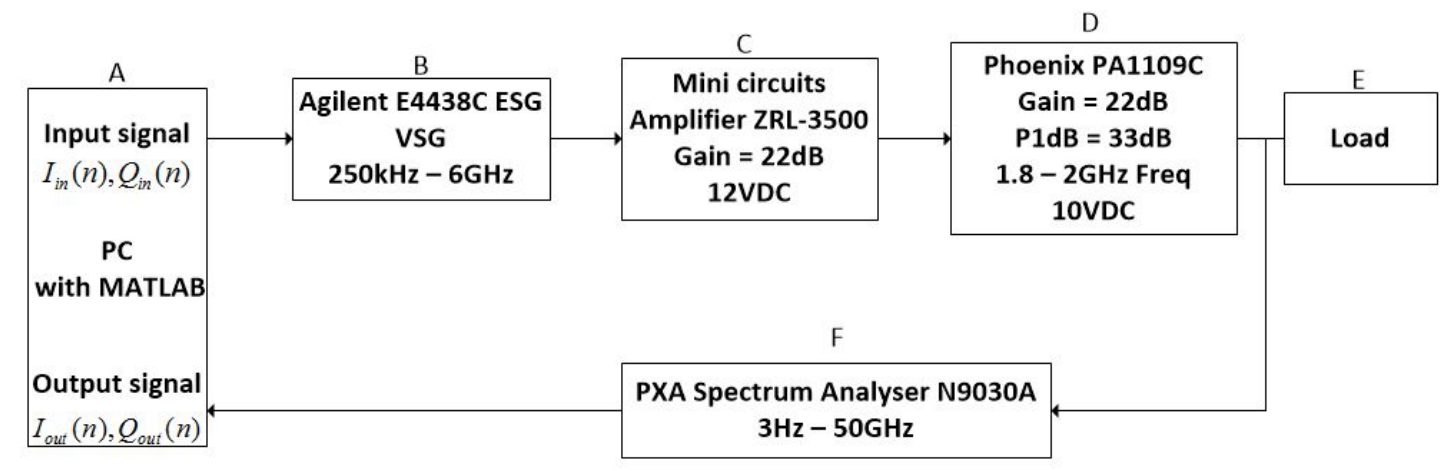

Figure 5.1: Block diagram of the experimental platform setup

- A - The PC running MATLAB simulation software. It generates complex baseband PA input excitation signals and uploads the data to the vector signal generator. The output data sequence is downloaded to the PC with a spectrum analyser (SA) data acquisition software.

- B - The Agilent E4438C ESG Vector signal generator (VSG) with $250 \mathrm{kHz}$ $6 \mathrm{GHz}$ instantaneous bandwidth. The VSG converts the digital input signal fed from the PC to analog signals and up-converts the PA input to a bandpass signal. The signal generated from the VSG is fed to a linear driver amplifier.

- C - The driver amplifier is fed with the up-converted analog signal from the VSG before it is sent to the Phoenix PA1109C DUT. The pre-amplifier prepares the signal for further processing by the PA.

- D - The DUT is a Phoenix PA1109C 10VDC PA operating at a frequency range of $1.8-2 \mathrm{GHz}$. The PA is supplied by a DC power source of $12 \mathrm{~V}$. The $1 \mathrm{~dB}$ compression point at the input of the $\mathrm{PA}$ is $33 \mathrm{~dB}$.

- E - A 6dB RF attenuator is placed at the DUT's output. The output signal is then connected to the spectrum analyser. 
- F - The PA output data are captured by the PXA spectrum analyser (SA). The analyser down-converts the analog signal from the PA and digitizes it into complex in-phase (I) and quadrature (Q) components of the bandpass signal.

The input-output characteristics of the DUT in the testbed setup is plotted and shown in Figure 5.2
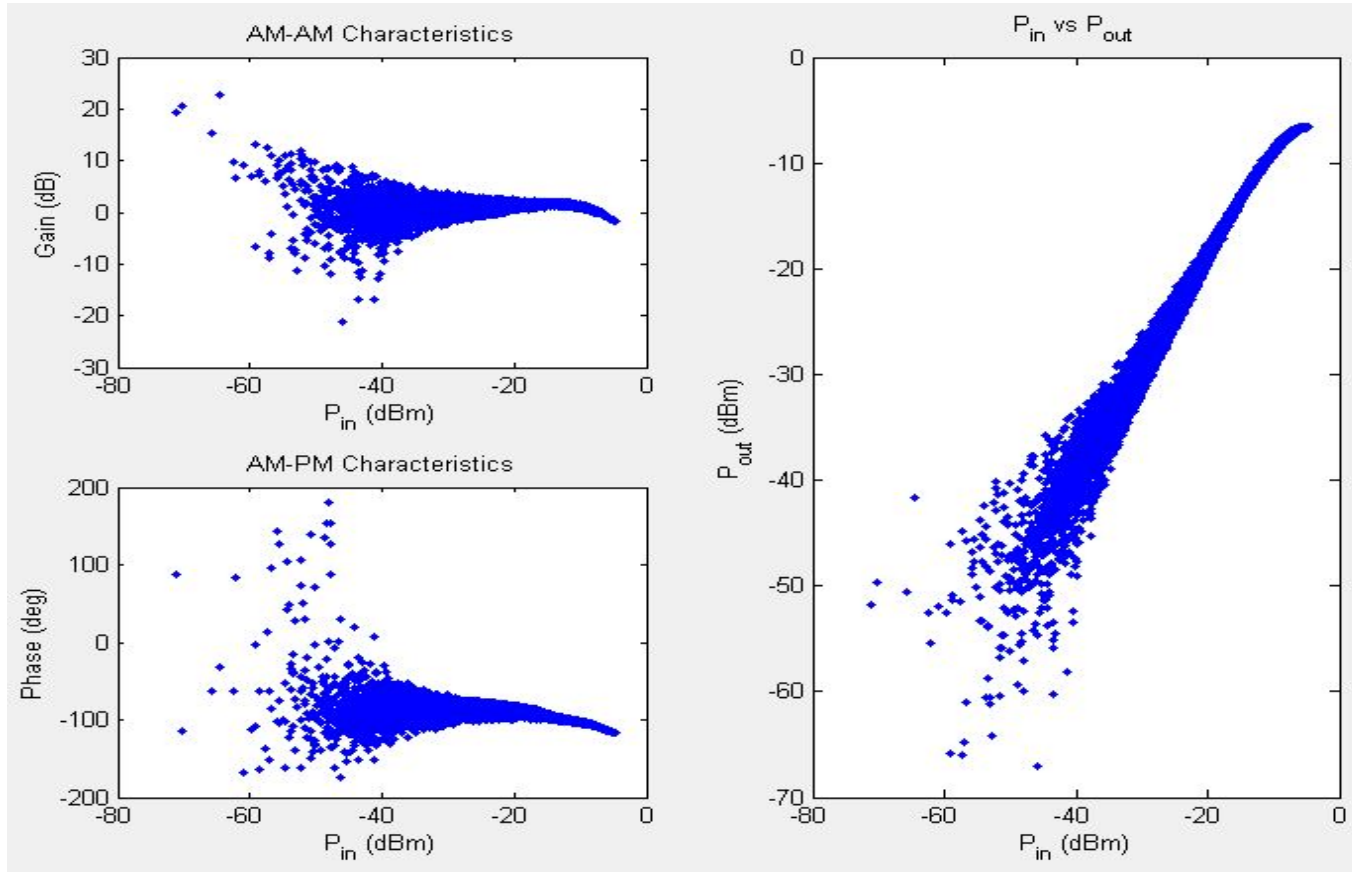

Figure 5.2: Transfer characteristics of the driver amplifier in cascade with the PA in the testbed setup

\subsection{Data Measurement Procedure}

A practical scenerio to be considered is a basestation PA experiencing signals with rapidly changing bandwidth sizes and power levels. Figure 5.3 illustrates a typical basestation PA excitation over a short period of time. To simulate a practical scenario that a PA in a basestation can experience, the PA in the experimental platform 


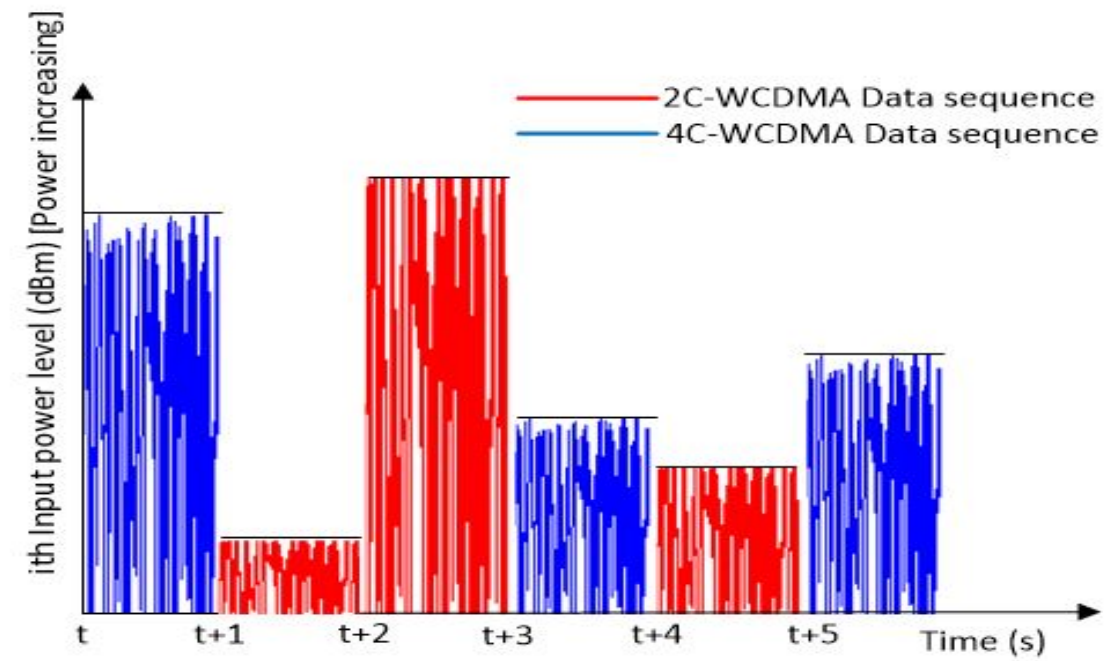

Figure 5.3: Changing input signals experienced by a PA over time

explained in the previous section, is excited by multicarrier WCDMA baseband signals with varying bandwidth and power levels. The input signals are:

- complex two-carrier (2C) WCDMA with a PAPR of 7.49dB and total bandwidth of $10-\mathrm{MHz}$ sampled at 100Mbps.

- complex four-carrier (4C) WCDMA signals (1111 carrier configuration - all carriers are used) with a PAPR of $7.64 \mathrm{~dB}$ and total bandwidth of $20-\mathrm{MHz}$ sampled at $100 \mathrm{Mbps}$.

The amplitude setting of the input signal in the VSG is varied (reference power level) to drive the PA over a large dynamic range (linear and nonlinear modes). The input signal set at different ith (high and low) average power levels is used to drive the PA. In order to protect the amplifier and the system from overheating, the maximum peak amplitude of the input signal at both bandwidths is limited to $-10.5 \mathrm{dBm}$. A million $I / Q$ input data samples are uploaded and the corresponding output data are downloaded. 
The datasets (input and corresponding output at an ith power level) are grouped and labelled for simplicity. Table 5.1 is a list of all the datasets measured at specific ith power levels used in simulations. The ith input amplitude setting is the power level of the input signal adjusted at the ESG Vector signal generator shown in Figure 5.1. The output power is the measured average power level of the output signals downloaded from the PC.

Figure 5.4 shows the power spectrum density (PSD) plots of the two- and fourcarrier WCDMA input and output datasets at different power levels. The legends in the plots correspond to the dataset, amplitude settings and carrier type listed in Table 5.1 The lowest traces in Figures 5.4(a) and 5.4(b) are the input signals with over $60 \mathrm{~dB}$ dynamic range. The output traces show the spectral regrowth caused by the amplifier, which is as high as $-25 \mathrm{~dB}$. The output traces are the plots of the original measured output signals shifted by a scaling factor defined in Section 4.5 (small signal gain). This is done to align the output trace with the input signal trace for convenience in displaying PSD plots. The output plots of all other figures hereafter shown in this chapter are shifted for convenience in displaying results. 
Table 5.1: List of measured input and output multicarrier signals at different ith power levels. The label $i$ is the index of the input signal's power level that specifies the $i t h$ operating condition. The label $\left(D_{i}\right)$ represents the index of the dataset (input and corresponding output) with a carrier size and at a specific ith power level setting

\begin{tabular}{|c|c|c|c|}
\hline Dataset $\left(D_{i}\right)$ & Test signal type & $\begin{array}{c}\text { ith Input } \\
\text { amplitude setting }(\mathrm{dBm})\end{array}$ & Output Power (dBm) \\
\hline 1 & 2C-WCDMA & -10.5 & -25.31 \\
\hline 2 & 2C-WCDMA & -11.5 & -25.98 \\
\hline 3 & 2C-WCDMA & -12.5 & -26.69 \\
\hline 4 & 2C-WCDMA & -13.5 & -27.44 \\
\hline 5 & 2C-WCDMA & -14.5 & -28.33 \\
\hline 6 & 2C-WCDMA & -15.5 & -29.26 \\
\hline 7 & 2C-WCDMA & -16.5 & -30.17 \\
\hline 8 & 2C-WCDMA & -17.5 & 31.13 \\
\hline 9 & 2C-WCDMA & -18.5 & -32.12 \\
\hline 10 & 4C-WCDMA & -10.5 & -25.37 \\
\hline 11 & 4C-WCDMA & -11.5 & -26.01 \\
\hline 12 & 4C-WCDMA & -12.5 & -26.72 \\
\hline 13 & 4C-WCDMA & -13.5 & -27.51 \\
\hline 14 & 4C-WCDMA & -14.5 & -28.38 \\
\hline 15 & 4C-WCDMA & -15.5 & -29.29 \\
\hline 16 & 4C-WCDMA & -16.5 & -30.22 \\
\hline 17 & 4C-WCDMA & -17.5 & -31.19 \\
\hline 18 & 4C-WCDMA & -18.5 & -32.17 \\
\hline 19 & 4C-WCDMA & -19.5 & -33.17 \\
\hline
\end{tabular}




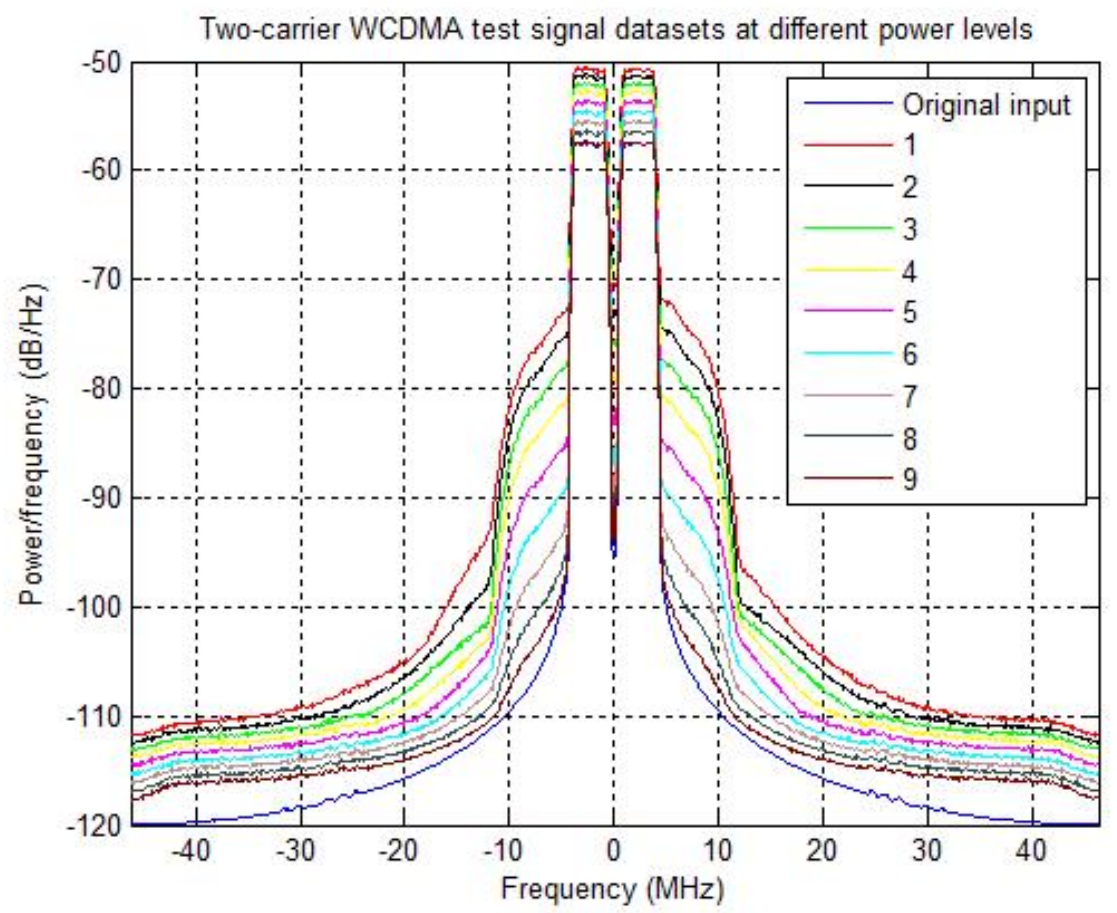

(a)

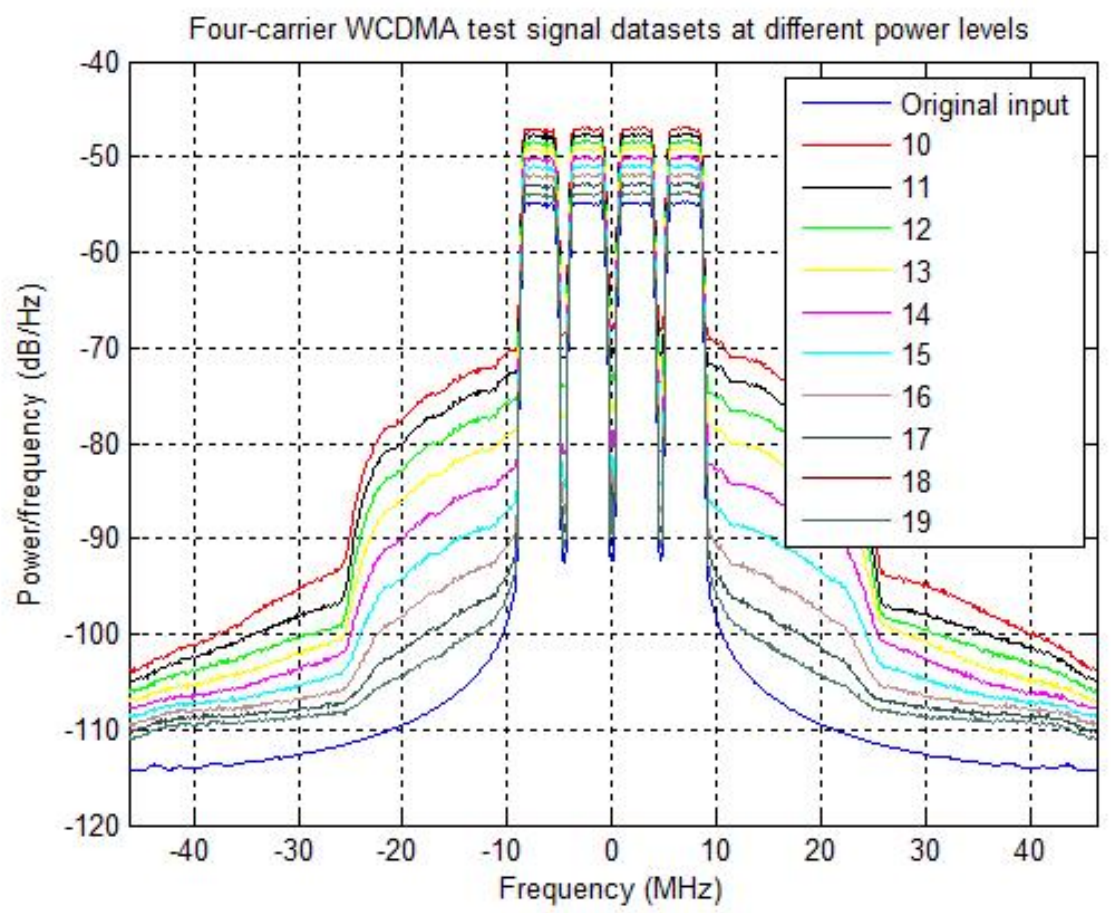

(b)

Figure 5.4: PSD plots of measured input and output (a) two-carrier WCDMA and (b) four-carrier WCDMA signals for various amplitude settings (legends correspond to ith values in Table 5.1) 


\subsection{Time Alignment Results}

The time delay estimation and alignment of the datasets is achieved using the AMPS software. This software applies the time alignment algorithm presented in [51] to the measured datasets. Figure 5.5 shows the graphical user interface (GUI) of the AMPS software. The file path of the baseband $I$ and $Q$ files can be set in the interface. The settings used for the alignment are chosen as:

- Sampling frequency - $100 \mathrm{MHz}$

- Interpolation rate - default value of 25

- Interpolation order - default value of 3 and

- Block size for calculating the cross-covariance - default value of 1000

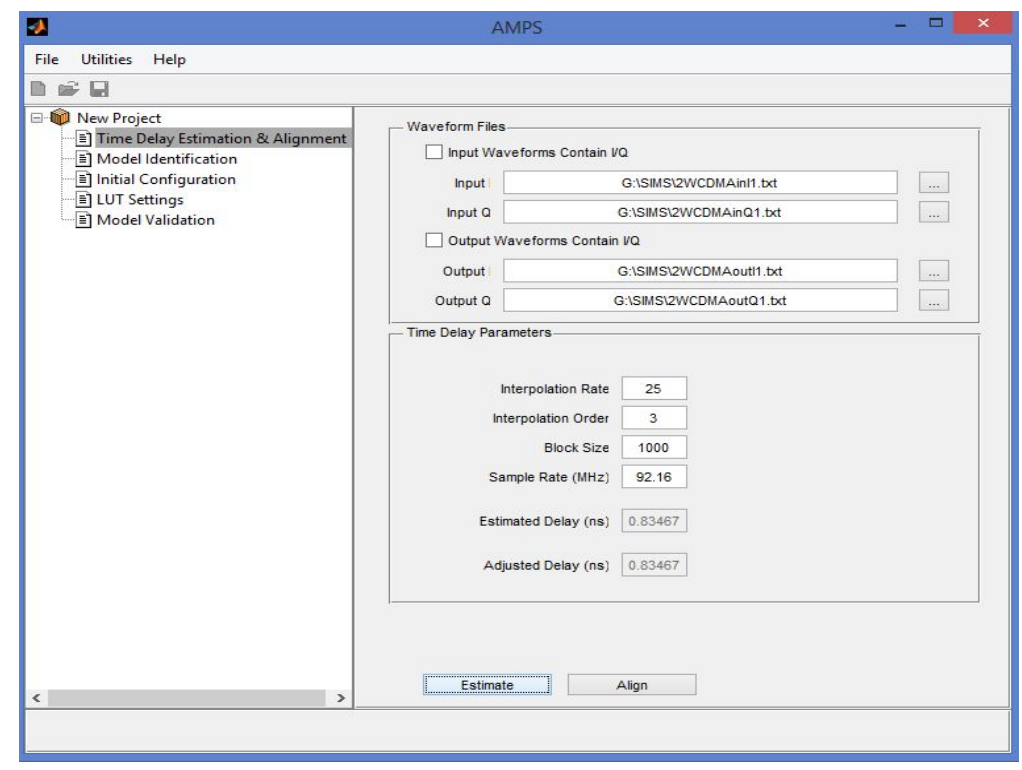

Figure 5.5: The GUI of the AMPS software for time delay estimation and alignment

The time delay estimation and alignment result of a two-carrier WCDMA signal at $-10.5 \mathrm{dBm}$ amplitude setting from the AMPS software is displayed in Figure 5.6. 

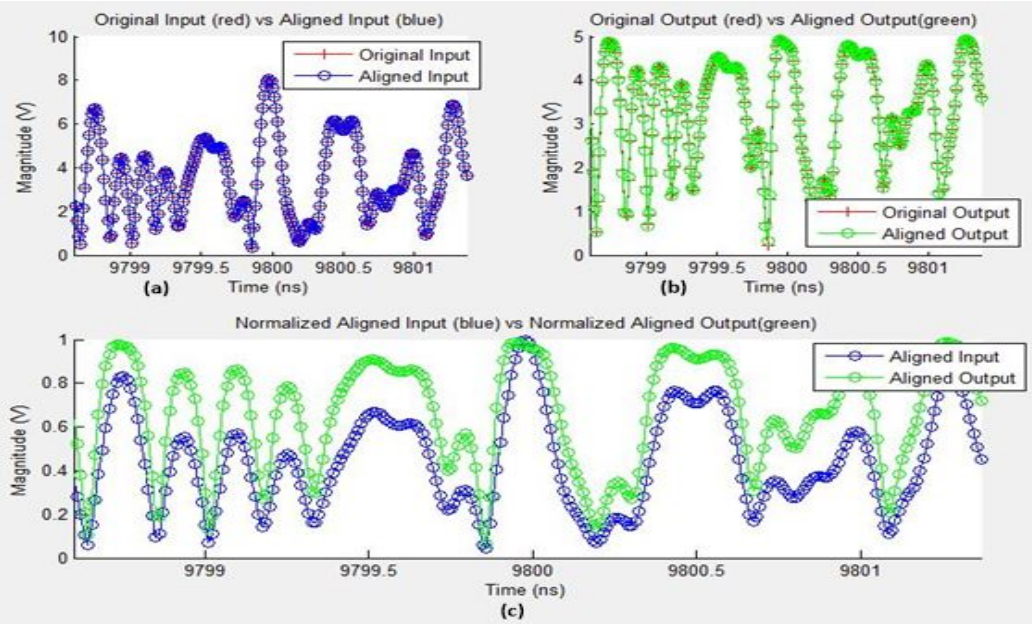

Figure 5.6: Comparison between the time domain plot of (a) the original measured input and the aligned input (b) the original measured output and the aligned output and (c) the aligned input and the output signal of a 2C-WCDMA signal

\subsection{PA Characterization}

The input and output signals measured are used to construct the AM/AM and AM/PM responses of the PA at any ith operating condition. Figure 5.7 and 5.8 show the AM/AM and AM/PM characteristic plots of the PA for the two- and fourcarrier WCDMA datasets with different bandwidths and input power levels. The $\mathrm{AM} / \mathrm{AM}$ and $\mathrm{AM} / \mathrm{PM}$ plots for different datasets show the dependence of the PA's operating point on the statistics (bandwidth and power level) of the input signal.

The increased dispersions observed in the characteristic plot for the 4C-WCDMA signal compared to that of the 2C-WCDMA shows the increase in memory effect on the PA as a result of an increase in the bandwidth of the signal. The plots for high $(-10.5 \mathrm{dBm})$ and low $(-18.5 \mathrm{dBm})$ power levels show the increase in the severity of the PA's nonlinearity as the input power level increases. This confirms the idea that the PA behavior is different for different input signals. 


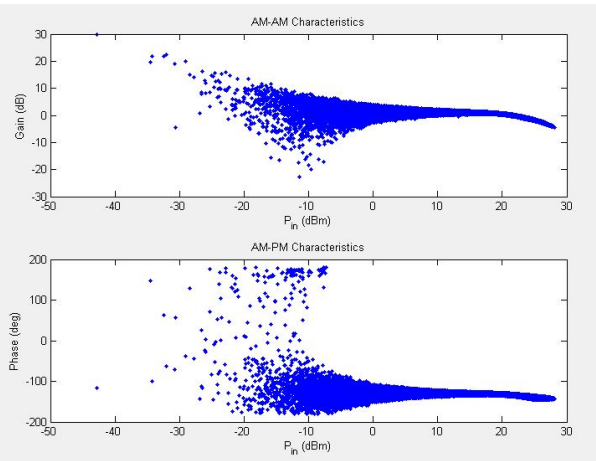

(a)
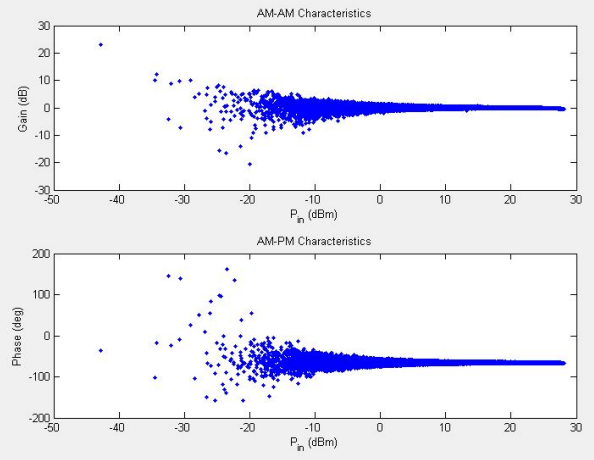

(b)

Figure 5.7: $\mathrm{AM} / \mathrm{AM}$ and $\mathrm{AM} / \mathrm{PM}$ response when the $\mathrm{PA}$ is excited by a two-carrier WCDMA signal at an input power level of (a) $-10.5 \mathrm{dBm}$ and (b) $-18.5 \mathrm{dBm}$

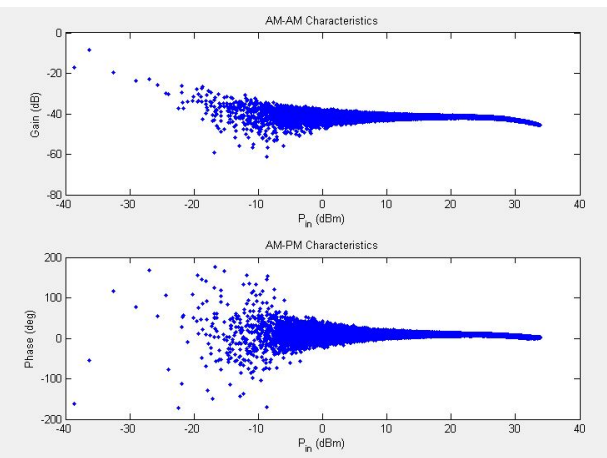

(a)

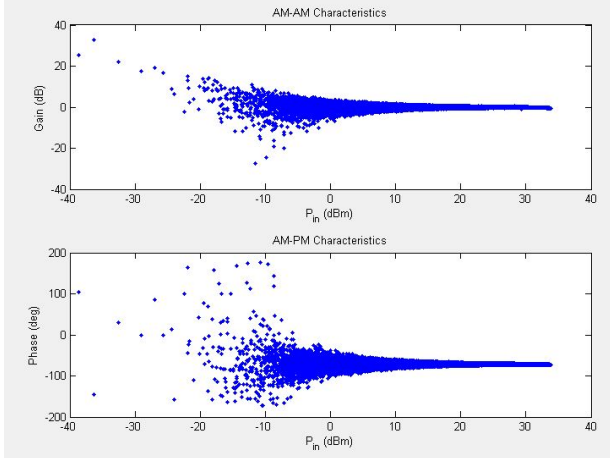

(b)

Figure 5.8: $\mathrm{AM} / \mathrm{AM}$ and $\mathrm{AM} / \mathrm{PM}$ response when the PA is excited by a four-carrier WCDMA signal at an input power level of (a) $-10.5 \mathrm{dBm}$ and (b) $-18.5 \mathrm{dBm}$

The ACPR for the original input and output signals measured are computed. The adjacent channel offsets of a WCDMA signal are typically $5 \mathrm{MHz}, 10 \mathrm{MHz}$, and $15 \mathrm{MHz}$ from the carrier. Offsets are measured from the first carrier for lower adjacent channel and the last carrier for the upper adjacent channel. The ACPR of the input signal at a particular bandwidth is constant for all power levels. The worst case ACPR in the $+5 \mathrm{MHz}$ or $-5 \mathrm{MHz}$ channel offset of the output signal is considered for evaluating the linearization performance. Table 5.2 lists the ACPR $( \pm 5 \mathrm{MHz}$ offset $)$ calculated for 
the input and output datasets measured from the PA before linearization (without a DPD). The measured output signal does not have equal ACPR at $\pm 5 \mathrm{MHz}$ offset

Table 5.2: ACPR values of measured datasets for simulation

\begin{tabular}{|c|c|c|c|}
\hline \multirow{2}{*}{ Dataset No. } & Input ACPR $(\mathrm{dBc})$ & \multicolumn{2}{|c|}{ Output ACPR $(\mathrm{dBc})$} \\
\cline { 2 - 4 } & $\pm 5 \mathrm{MHz}$ & $-5 \mathrm{MHz}$ & $+5 \mathrm{MHz}$ \\
\hline 1 & -62.82 & -36.07 & -35.48 \\
\hline 2 & -62.82 & -38.01 & -37.45 \\
\hline 3 & -62.82 & -40.20 & -39.69 \\
\hline 4 & -62.82 & -42.79 & -42.27 \\
\hline 5 & -62.82 & -46.18 & -45.78 \\
\hline 6 & -62.82 & -49.35 & -49.22 \\
\hline 7 & -62.82 & -52.38 & -52.33 \\
\hline 8 & -62.82 & -54.75 & -54.74 \\
\hline 9 & -62.82 & -56.33 & -55.80 \\
\hline 10 & -53.77 & -24.25 & -23.06 \\
\hline 11 & -53.77 & -26.00 & -25.00 \\
\hline 12 & -53.77 & -28.15 & -27.28 \\
\hline 13 & -53.77 & -30.82 & -30.08 \\
\hline 14 & -53.77 & -34.00 & -33.51 \\
\hline 15 & -53.77 & -37.42 & -37.17 \\
\hline 16 & -53.77 & -40.88 & -40.73 \\
\hline 17 & -53.77 & -44.13 & -44.08 \\
\hline 18 & -53.77 & -46.65 & -46.57 \\
\hline 19 & -53.77 & -48.51 & -48.40 \\
\hline
\end{tabular}

as observed from the asymmetrical output spectrum plot of Figure 5.4 which further confirms that a PA with memory nonlinearity generates an asymmetrical output spectrum plot.

\subsection{Static DPD Simulation}

The performance of a static DPD using the particular datasets measured is evaluated. The results from the static DPD simulation is used to: 
- Verify the effect of the nonlinear and memory order of a model structure on the modeling accuracy, and the DPD performance.

- Compare the linearization performance of signals at the same power levels and modulation but different bandwidth.

- Compare the linearization performance for signals with same modulation, same bandwidth but different power levels.

- Study the poor linearization performance of a static DPD for a PA experiencing rapid signal changes.

\subsubsection{PA and DPD Modeling Simulation}

The accuracy of the PA and DPD model is evaluated using the NMSE between the estimated model output from simulation, and the original measured input. An unbiased estimate of the PA and DPD will proportionally improve the overall linearization performance. The modeling accuracy of an estimation at a particular configuration can be affected by the:

- varying bandwidth sizes of signals at a fixed power level and

- varying power levels of signals at fixed bandwidth

The choice of the model configuration also has a significant effect on the modeling accuracy of an estimation.

\section{Fixed Power Level, Varying Bandwidth and Model Configurations}

Different configurations (polynomial order $K$ and memory depth order $M$ ) of the memory polynomial model, for the PA and DPD estimation, are tested. We use 1000 samples to extract the parameters of the PA and DPD model for different 
configuration settings. Table 5.3 lists the NMSE associated with estimating the PA and DPD model for different model configurations using the two- and four-carrier WCDMA signals at $-10.5 \mathrm{dBm}$.

Table 5.3: Modeling accuracy results from the PA and DPD modeling for different configurations using 2C-WCDMA and 4C-WCDMA at the same power level. The number of parameters estimated from each configuration is represented as $K M$.

\begin{tabular}{|l|c|c|c|c|c|c|}
\hline \multicolumn{3}{|c|}{ Configuration } & \multicolumn{2}{|c|}{ PA NMSE (dB) } & \multicolumn{2}{c|}{ DPD NMSE (dB) } \\
\hline$K$ & $M$ & $K M$ & 2C-WCDMA & 4C-WCDMA & 2C-WCDMA & 4C-WCDMA \\
\hline 3 & 1 & 3 & -29.00 & -23.73 & -26.13 & -23.16 \\
3 & 2 & 6 & -35.55 & -33.51 & -28.55 & -28.25 \\
5 & 2 & 10 & -37.32 & -35.05 & -30.56 & -30.38 \\
5 & 3 & 15 & -38.68 & -36.63 & -31.31 & -31.74 \\
5 & 4 & 20 & -39.05 & -36.81 & -31.51 & -31.82 \\
7 & 3 & 21 & -38.86 & -36.86 & -31.52 & -32.01 \\
9 & 3 & 27 & -39.00 & -36.93 & -31.60 & -32.12 \\
9 & 5 & 45 & -39.94 & -37.47 & -31.99 & -32.56 \\
13 & 5 & 65 & -40.31 & -37.61 & -32.05 & -32.79 \\
\hline
\end{tabular}

The PA and DPD estimated NMSE values decrease as the configuration order increases until a certain configuration where the NMSE remains constant regardless of any further increase in configuration. It is observed that higher model configurations are required with the 4C-WCDMA signal to achieve an equivalent modeling accuracy derived for a 2C-WCDMA signal at the same power level. The 4C-WCDMA signal requires a configuration of $K=5, M=3$ to achieve the acceptable NMSE while this can be achieved at $K=3, M=2$ for the 2C-WCDMA signal at the same power level. Figure 5.9 illustrates the relationship between the accuracy (NMSE) of an estimated 
model and the number of estimated model parameters.

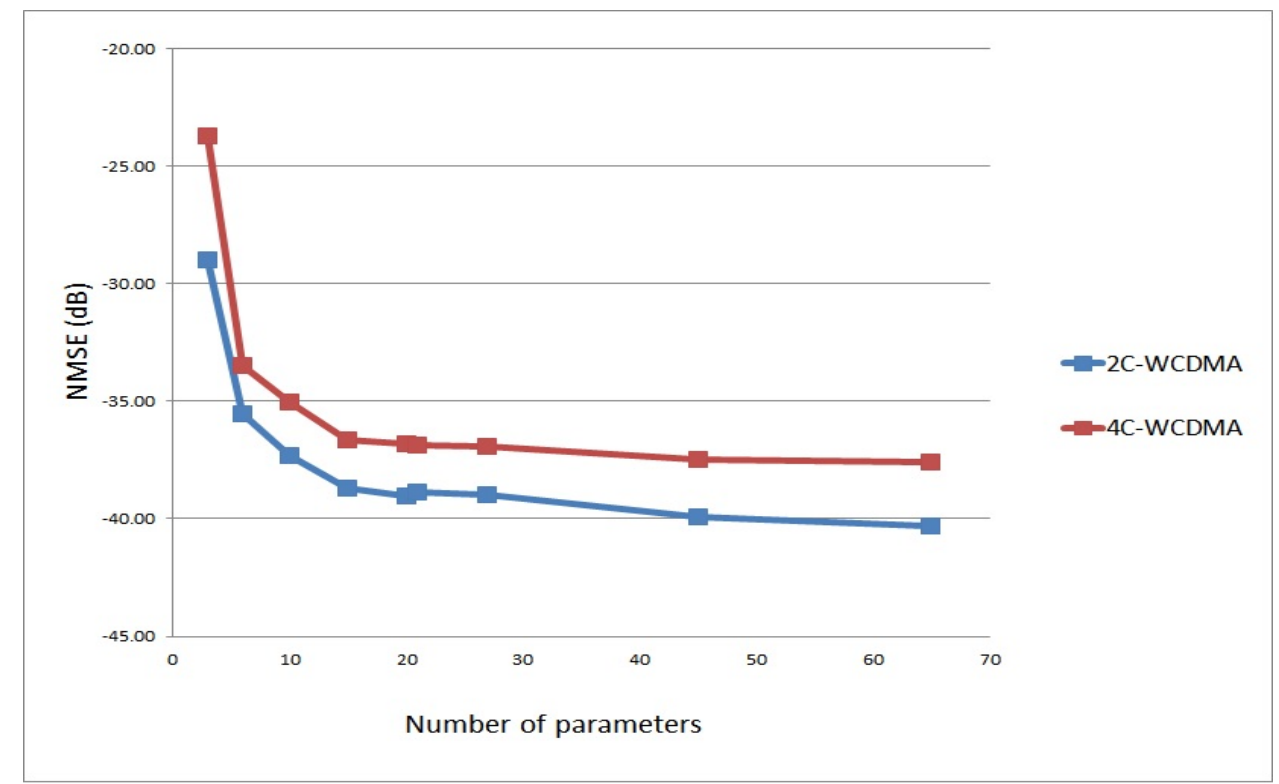

Figure 5.9: NMSE values compared to the number of parameters used to model a PA and DPD for a two-carrier(blue) and a four-carrier (red) WCDMA signal at an ith power level

\section{Varying Power Level, Fixed Bandwidth and Model Configuration}

Modeling of the PA and DPD with high powered signals require higher model configurations than with low powered signals. An increase in the configuration settings does not guarantee an improvement in the linearization performance and modeling accuracy. This is because a certain configuration is adequate to capture the nonlinear and memory effects of the PA for the highest powered signal that can excite a PA. Table 5.4 shows the accuracy of the models estimated for the two-carrier WCDMA signal at different power levels using a fixed model configuration of $K=5$ and $M=3$. The NMSE value reduces as the power level of the signal reduces for modeling with the same configuration. The nonlinear order of a model for estimating the behavior of a PA and DPD increases with the power level of the dataset until acceptable accuracy 
Table 5.4: Comparison of the PA and DPD modeling accuracy with a fixed configuration for the 2C-WCDMA signal at different power levels

\begin{tabular}{c|c|c}
\hline Dataset & PA NMSE (dB) & DPD NMSE (dB) \\
\hline \hline 2C-WCDMA @ -10.5dBm & -38.68 & -31.31 \\
2C-WCDMA @ -11.5dBm & -41.53 & -35.08 \\
2C-WCDMA @ -12.5dBm & -43.63 & -38.96 \\
2C-WCDMA @ -13.5dBm & -45.17 & -42.57 \\
2C-WCDMA @-14.5dBm & -46.04 & -44.89 \\
2C-WCDMA @-15.5dBm & -45.74 & -44.96 \\
2C-WCDMA @ -16.5dBm & -46.02 & -45.22 \\
\hline
\end{tabular}

is achieved. The memory depth order required for a model also increases with the bandwidth of the dataset.

The computational complexity for estimating the model increases with an increase in the configuration order because of the increase in the number of parameters to be estimated. Therefore, a trade-off between accuracy and complexity has to made. The least configuration possible to achieve the acceptable performance is usually a good choice if complexity of implementation is of high importance.

\subsubsection{Static DPD Linearization Performance}

The static DPD is simulated using the procedure outlined in Chapter 4 and its linearization performance is evaluated. The case of a PA experiencing input signal changes is considered to study the poor performance of a static DPD (Figure 5.10). At a time $t$, the PA is excited by a $-10.5 \mathrm{dBm} 4 \mathrm{C}$-WCDMA signal. The DPD at this time $t$ is modeled using 1000 data samples from the 4C-WCDMA dataset. Figure 5.11(a) shows the PSD plots of the original input, original output without DPD and the output after the static DPD is applied to a PA excited by a 4C-WCDMA signal at a specific time $t$. The output spectrum regrowth shown as the red trace was significantly suppressed by about $25 \mathrm{~dB}$ to give the green trace in Figure 5.11(a). 


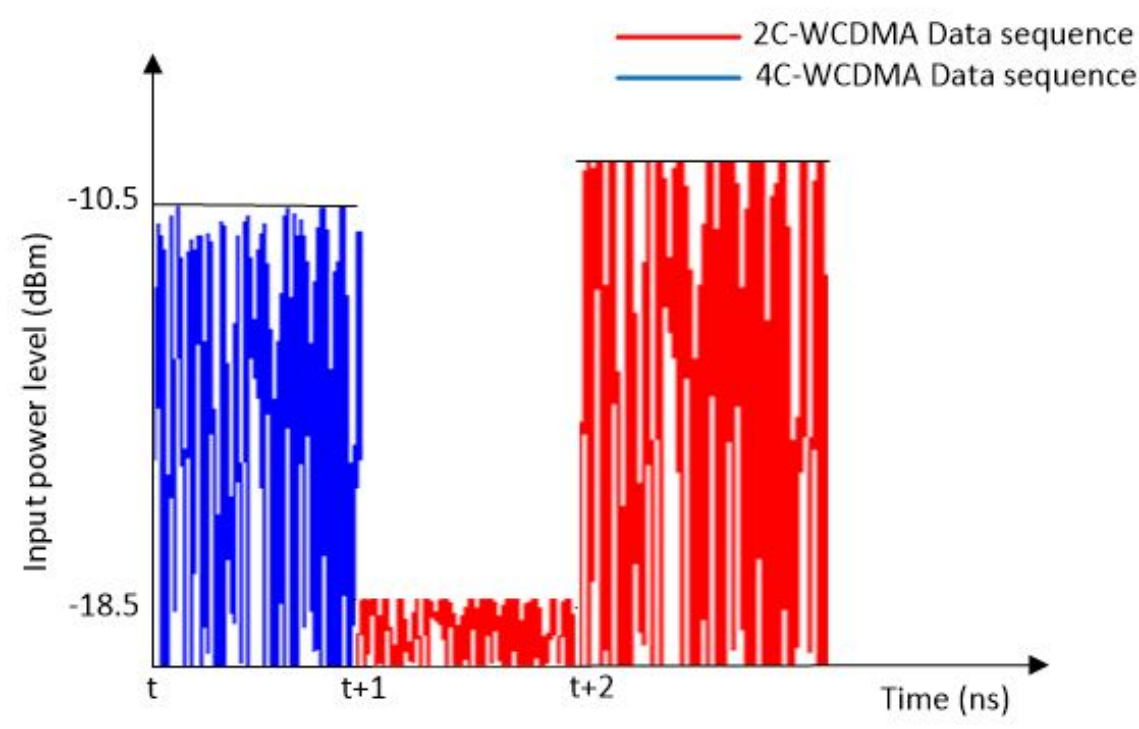

Figure 5.10: Input signal changes experienced by a $\mathrm{PA}$ at time $\mathrm{t}, \mathrm{t}+1$, and $\mathrm{t}+2$

At a time $t+1$, the input signal to the PA changes to a $-18.5 \mathrm{dBm} 2 \mathrm{C}-\mathrm{WCDMA}$ signal and at time $t+2$, the PA input becomes a $-10.5 \mathrm{dBm} 2 \mathrm{C}$-WCDMA signal. The performance of the static DPD for the input signals at time $t+1$ and $t+2$ is shown in Figure 5.11(b) and 5.11(c). An undesired spectrum regrowth increase (red to green trace) is observed in Figure 5.11(b) and a 10dB spectrum regrowth reduction in Figure 5.11(c). Thus, a poor linearization performance is observed for any other dataset different from the dataset $(-10.5 \mathrm{dBm}$ 4C-WCDMA signal in this case) used to model the static DPD.

The static DPD cannot adapt to the new data at any time $\geq t+1$. In practice, samples from the new dataset are measured and utilized to re-train the DPD. The static DPD scheme experiences large transient errors and increased computation complexity. Table 5.5 lists the linearization performance for a static DPD trained once at a time $\mathrm{t}$ in comparison with a static DPD re-trained at every change in input signal. A good linearization performance is only expected when the DPD experiences 
the signal it was trained with.

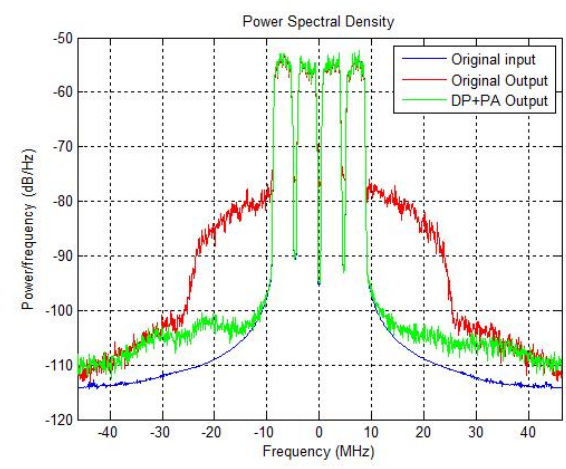

(a)

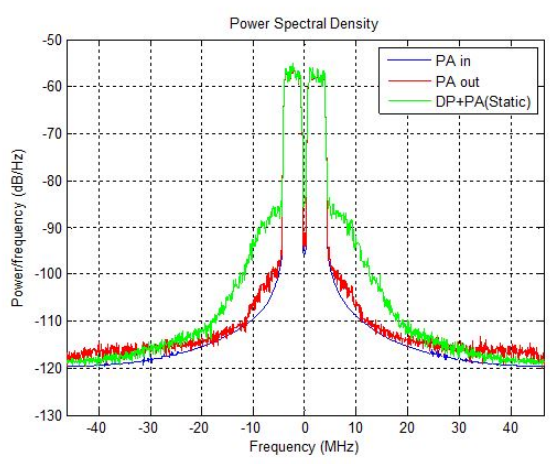

(b)

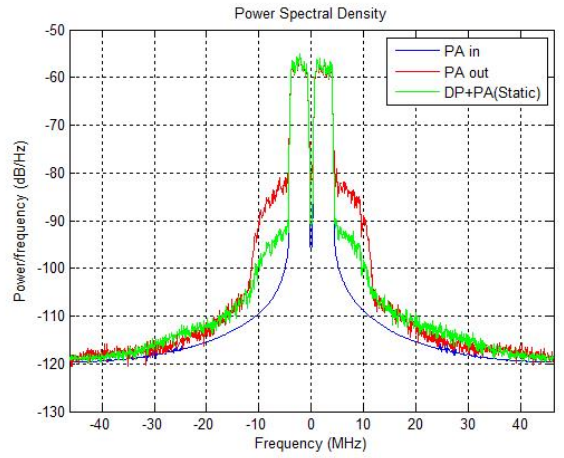

(c)

Figure 5.11: Linearization performance of a static DP for a PA excited by (a)$10.5 \mathrm{dBm} 4 \mathrm{C}$-WCDMA at $t$ (b) $-18.5 \mathrm{dBm} 2 \mathrm{C}$-WCDMA at $t+1$ (c) $-10.5 \mathrm{dBm}$ $2 \mathrm{C}-\mathrm{WCDMA} t+2$

Table 5.5: Comparison of the ACPR and NMSE of the PA output with a DPD modeled with datasets at different times and the PA output with a static DPD modeled using the signal at only time $t$

\begin{tabular}{c|c|c|c}
\hline Time & Dataset & NMSE $(\mathrm{dB})$ & ACPR $(\mathrm{dBc})$ \\
\hline$t$ & 4C-WCDMA @ -10.5dBm & -41.66 & -47.77 \\
\hline \hline$t$ & 2C-WCDMA @ $-18.5 \mathrm{dBm}$ & -25.38 & -40.46 \\
$t+1$ & 2C-WCDMA @ -18.5dBm & -56.02 & -53.67 \\
\hline \hline$t$ & 2C-WCDMA @ $-10.5 \mathrm{dBm}$ & -22.35 & -45.05 \\
$t+2$ & 2C-WCDMA @-10.5dBm & -44.03 & -51.78 \\
\hline
\end{tabular}




\subsubsection{Effect of Power Levels, Bandwidth and Model Config- uration on Linearization Performance}

The linearization performance of a DPD can be affected by the choice of the model configuration for signals at a particular power level and bandwidth size. The static DPD is simulated at different configurations for signals at different power levels and bandwidth sizes.

\section{Fixed Power Level, Varying Bandwidth}

The linearization performance of the static DPD is compared for the $2 \mathrm{C}$ - and $4 \mathrm{C}$ WCDMA signal at a fixed configuration and power level. Figure 5.12 shows the PSD plot of the linearized output (green traces) of the 2C-WCDMA and 4C-WCDMA signal at the same power level. The spectrum regrowth reduction in the $2 \mathrm{C}$-WCDMA output signal is observed to be higher than the reduction in the 4C-WCDMA output signal.

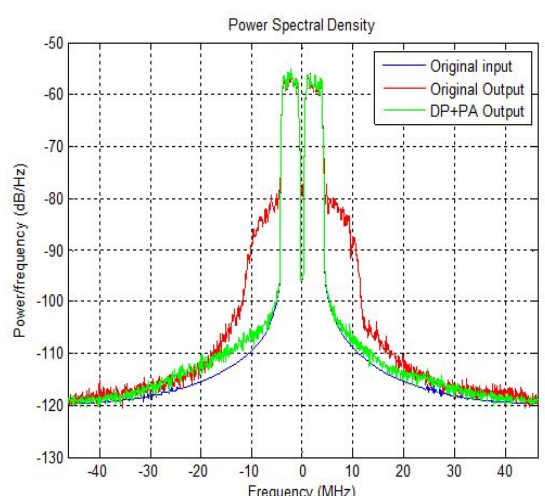

(a)

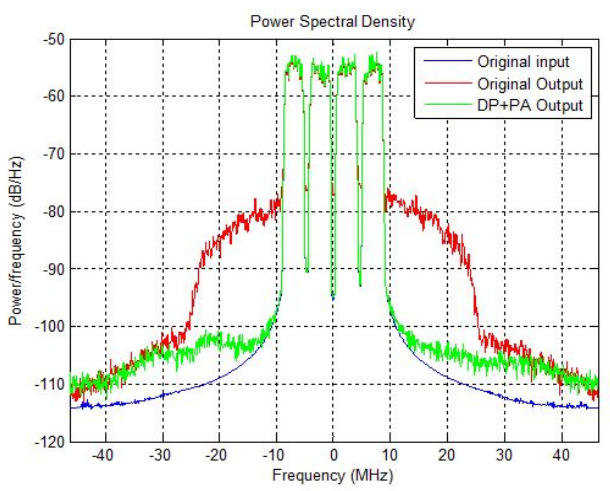

(b)

Figure 5.12: Comparison of the linearization performance of a static DPD with configuration $K=5, M=3$, for a PA excited by WCDMA signals at the same power level but different bandwidths (a)-10.5dBm 2C-WCDMA (b)-10.5dBm 4C-WCDMA 
The linearization performance of the static DPD is also compared at different configurations for the 2C-WCDMA and 4C-WCDMA signals at the same power level. Table 5.6 shows the ACPR and NMSE achieved for the 4C-WCDMA signal and 2CWCDMA signal at a fixed power level for different configurations. At a given power level, higher configuration orders are required for signals at a higher bandwidth to give comparable linearization performances when signals at a lower bandwidth are used.

Table 5.6: Linearization performance for varying model configurations using the 2C-WCDMA and 4C-WCDMA signal at $-10.5 \mathrm{dBm}$

\begin{tabular}{c|c|c|c}
\hline Model configuration & Dataset & NMSE $(\mathrm{dB})$ & $\mathrm{ACPR}(\mathrm{dBc})$ \\
\hline$K=3$ & 2C-WCDMA & -27.34 & -36.96 \\
\cline { 2 - 4 }$M=2$ & 4C-WCDMA & -27.14 & -31.89 \\
\hline$K=5$ & 2C-WCDMA & -44.03 & -51.78 \\
\cline { 2 - 4 }$M=3$ & 4C-WCDMA & -41.66 & -47.23 \\
\hline$K=7$ & 2C-WCDMA & -44.68 & -52.33 \\
\cline { 2 - 4 }$M=3$ & 4C-WCDMA & -42.02 & -47.77 \\
\hline
\end{tabular}

\section{Varying Power Levels, Fixed Bandwidth}

The linearization performance of the static DPD is compared at different configurations for the 2C-WCDMA or 4C-WCDMA signal for different power levels. Table 5.7 shows that the linearization performance increases as the model configuration is increased for a high powered signal. A poor performance of $-31.89 \mathrm{dBc}$ (above $-45 \mathrm{dBc}$ threshold) was observed for the $-10.5 \mathrm{dBm}$ 4C-WCDMA signal with a model configuration of $K=3, M=2$. At low order model configurations, the DPD is inadequate to successfully suppress the spectrum outgrowth for high powered signals. The PSD plots of the DPD+PA output for different model configurations for a high and low powered 2C-WCDMA and 4C-WCDMA signal is shown in Figures 5.13 and 5.14. 
Table 5.7: Linearization performance (ACPR in $\mathrm{dBc}$ ) for different configurations using the 4C-WCDMA signal at varying power levels

\begin{tabular}{c|c|c|c}
\hline Dataset & $K=3, M=2$ & $K=5, M=3$ & $K=7, M=3$ \\
\hline \hline 4C-WCDMA @ -10.5dBm & -31.89 & -47.77 & -47.23 \\
4C-WCDMA @ -11.5dBm & -35.81 & -50.83 & -50.50 \\
4C-WCDMA @ -12.5dBm & -40.13 & -52.20 & -52.61 \\
4C-WCDMA @ -13.5dBm & -45.16 & -52.43 & -53.23 \\
4C-WCDMA @ -14.5dBm & -50.01 & -52.94 & -53.43 \\
\hline
\end{tabular}

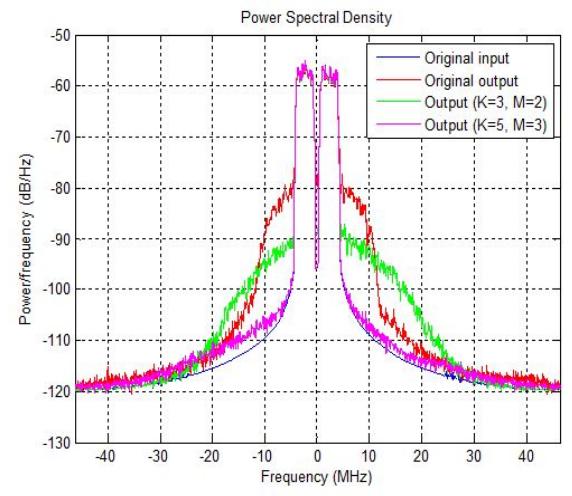

(a)

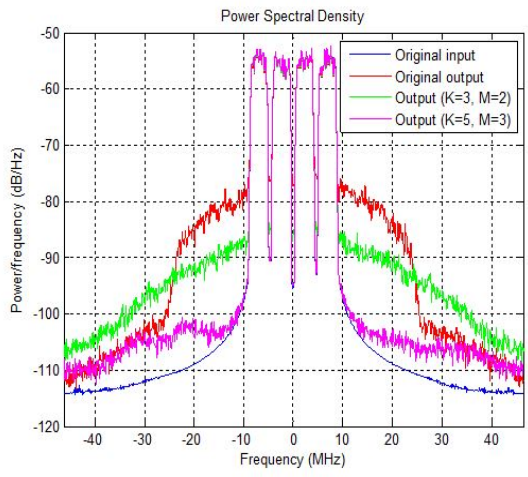

(b)

Figure 5.13: Comparison of the linearization performance of a static DPD with configuration $K=3, M=2$ and $K=5, M=3$, at $-10.5 \mathrm{dBm}$ (high powered signal) for (a) a 2C-WCDMA signal and (b) a 4C-WCDMA signal

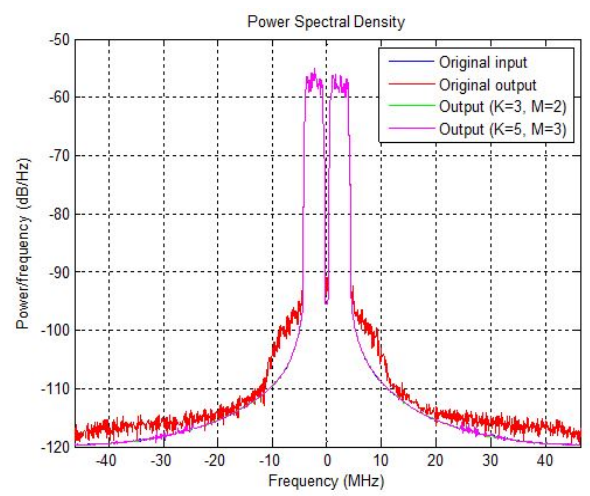

(a)

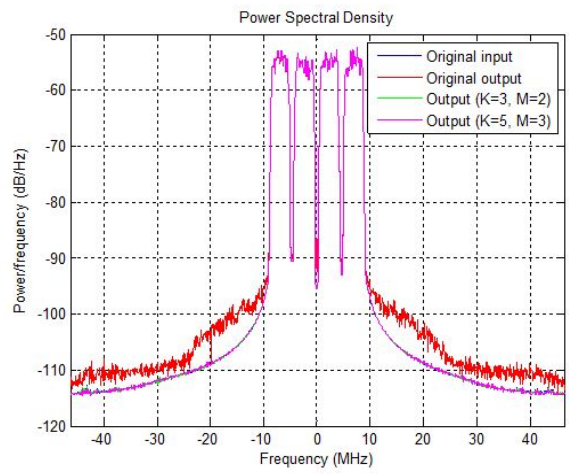

(b)

Figure 5.14: Comparison of the linearization performance of a static DPD with configuration $K=3, M=2$ and $K=5, M=3$, at $-17.5 \mathrm{dBm}$ (low powered signal) for (a) a 2C-WCDMA signal and (b) a 4C-WCDMA signal 
Acceptable performance is only achieved when the configuration increases for high powered signals. However, at this same configuration, the DPD using 4C-WCDMA signals with power levels lesser than $-12.5 \mathrm{dBm}$ (lower powered signals) can achieve good performance. Thus, low order DPD model configurations are adequate for the PA excited by low powered signals.

\subsection{First Order Linear Digital Pre-distortion}

Following from the results presented in Section 5.5 and shown in Figure 5.14, the PA behavior is close to linear for input signals at low power levels. A PA exhibiting mild nonlinearity can be linearized by a simple first order linear model-based DPD. A simple first order linear function is used as the PA and DPD model. The measured AM/AM and AM/PM characteristics of the PA are fitted to a linear first order model. The parameters of the model and its inverse are estimated using adaptive algorithms outlined in Chapter 3. Figure 5.15 is a block diagram of a $1 s t$ order linear model based pre-distorter.

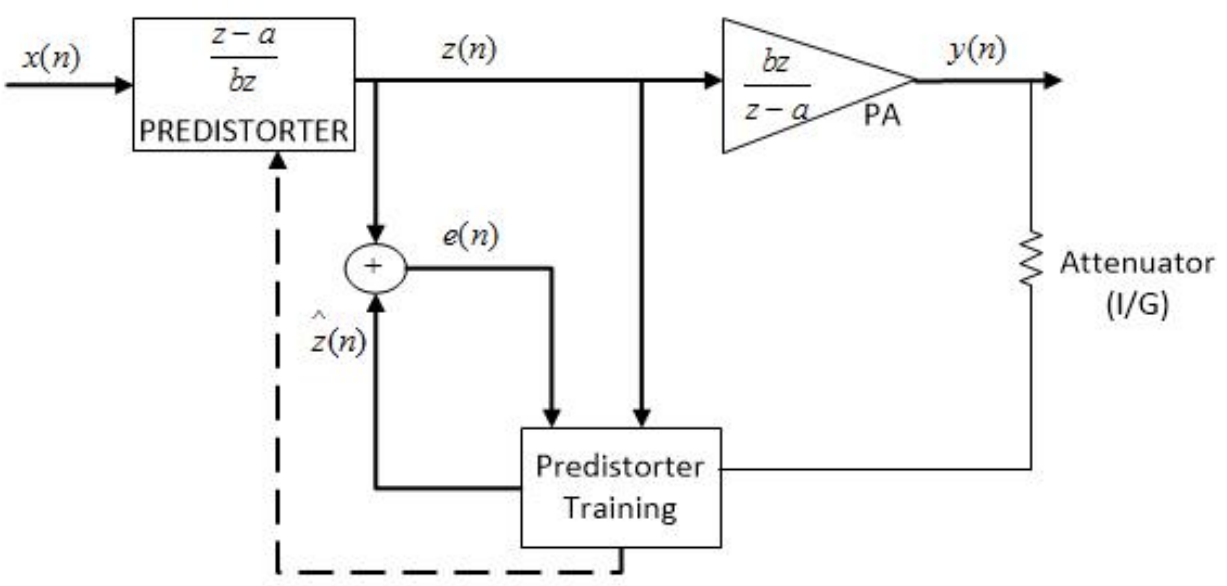

Figure 5.15: Block diagram of a first order linear adaptive DPD 
It is assumed that the PA behavior follows a 1st order linear transfer function:

$$
\frac{Y_{P A}(z)}{X_{P A}(z)}=\frac{b z}{z-a}
$$

where $Y_{P A}(z)$ and $X_{P A}(z)$ are the output and input of the PA respectively and the parameters of the model are the terms $a$ and $b$. Equation 5.1 can be re-written as

$$
y_{P A}(n)=a y_{P A}(n-1)+b x_{P A}(n)
$$

The PA's inverse function for the DPD is

$$
\frac{Y_{D P}(z)}{X_{D P}(z)}=\frac{z-a}{b z}
$$

where $Y_{D P}(z)$ and $X_{D P}(z)$ are the output and input of the DPD respectively. Equation 5.3 can also be re-written as

$$
y_{D P}(n)=\frac{1}{b} x_{D P}(n)-\frac{a}{b} x_{D P}(n-1)
$$

Figures 5.16(a) and 5.16(b) are time plots of the original output and estimated output for the PA and DPD modeling using a first order linear function. The high modeling accuracy is observed from the plot as the estimated output approximately equal to the original measured output. Table 5.8 lists the NMSE and ACPR of low powered 2Cand $4 \mathrm{C}$-WCDMA signals using the first order adaptive DPD. The very low values of the ACPR and NMSE in the table indicates the high linearization performance that can be achieved with the first order linear model-based adaptive DPD scheme. 
Table 5.8: First order linear model adaptive DPD performance for low powered WCDMA signals .

\begin{tabular}{c|c|c}
\hline \hline Signal @ -18.5dBm & NMSE (dB) & ACPR (dBc) \\
\hline \hline 2C-WCDMA & -63.52 & -62.76 \\
4C-WCDMA & -56.80 & -53.67 \\
\hline \hline
\end{tabular}

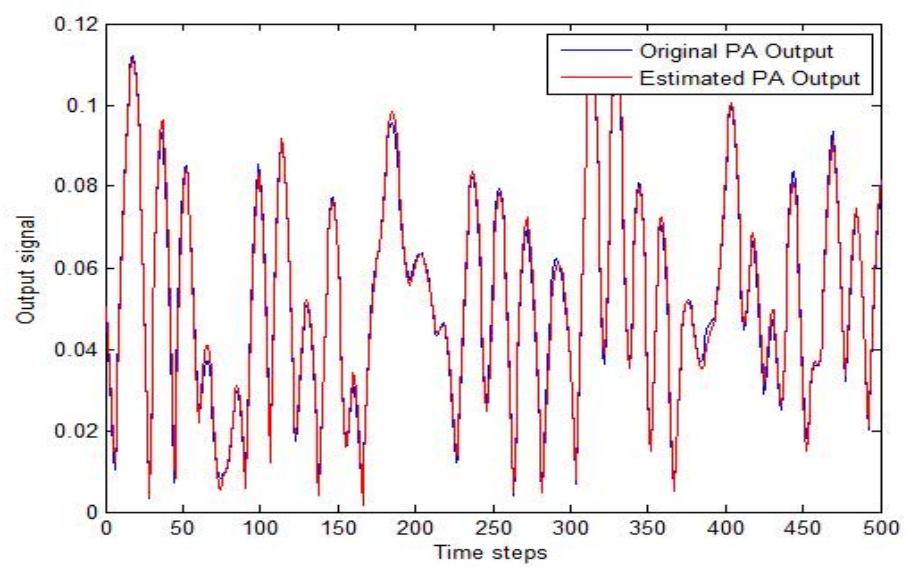

(a)

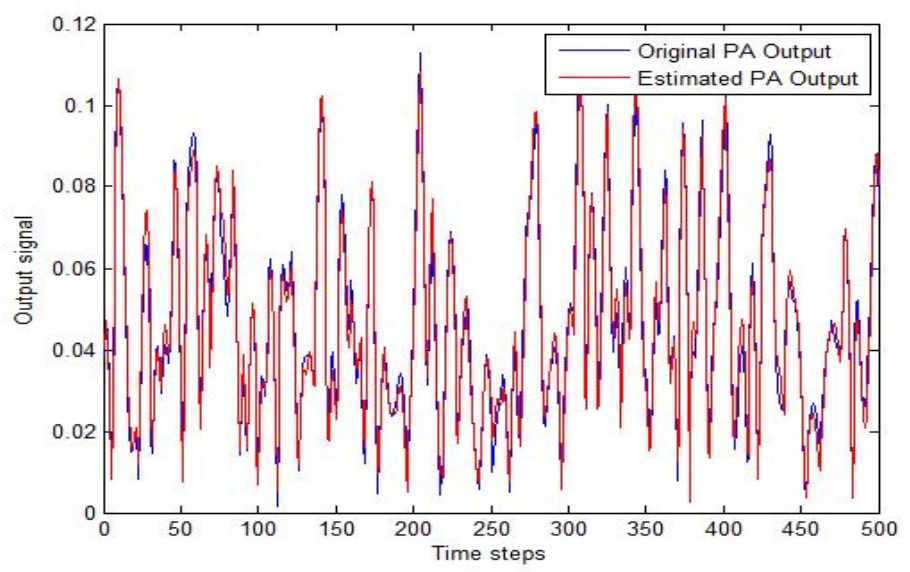

(b)

Figure 5.16: Time plot of the original output and estimated output of a modeled PA for a (a) two-carrier WCDMA signal (b) four-carrier WCDMA signal at $-18.5 \mathrm{dBm}$. 
The cascade of the first order DPD and PA is the original signal at the PA's input. Figure 5.17 shows the performance of a PA linearized with a first order linear model ADPD. The DPD is capable of reproducing the original PA input without any observable spectrum regrowth. Also, the first order linear model ADPD requires the estimation of only two parameters and provides a good linearization of the PA at low operating power condition at a relatively fast speed.

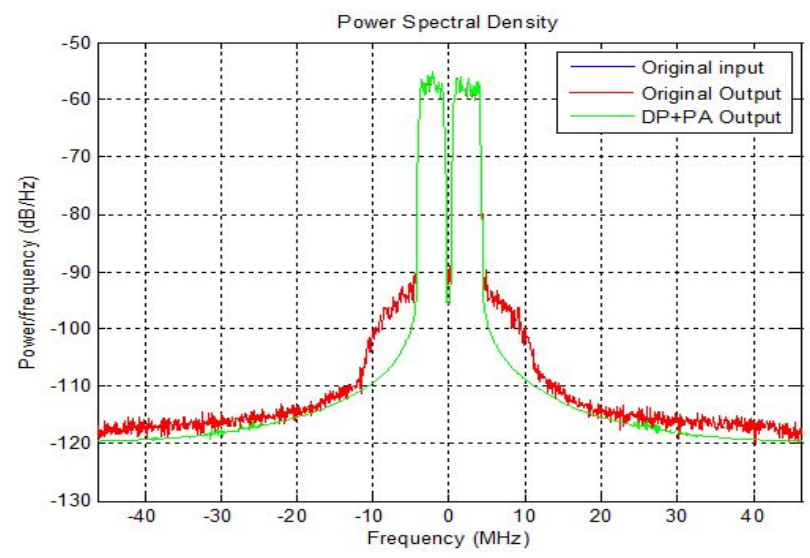

(a)

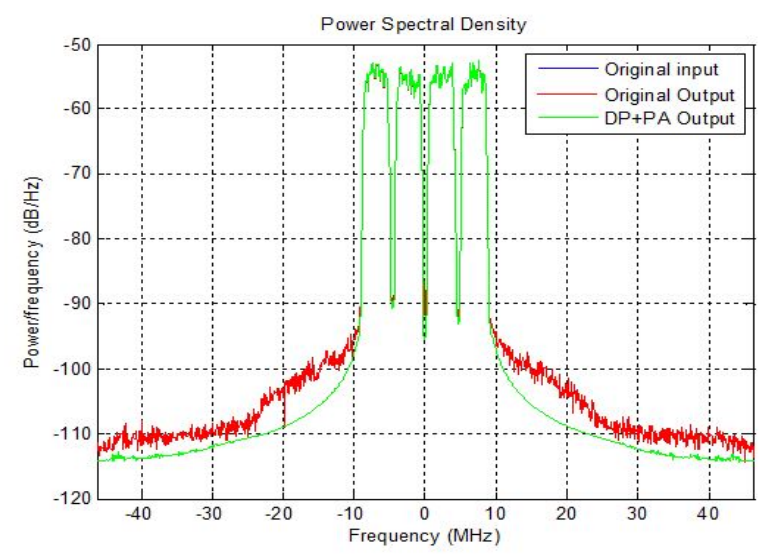

(b)

Figure 5.17: PSD plots of the original input, the original output before the DPD and the simulated DPD+PA output of a low powered (a) two-carrier WCDMA signal and (b) four-carrier WCDMA signal using the first order linear modelbased ADPD 
The implementation of the first order linear DPD is simpler and less complex than a nonlinear model DPD that can require estimation of more than two parameters. However, the first order model is not sufficient to compensate for the increased nonlinearity exhibited by the PA at a higher operating power level. Figure 5.18 shows the minimal spectral regrowth reduction for output signal for a PA at a high operating power condition.

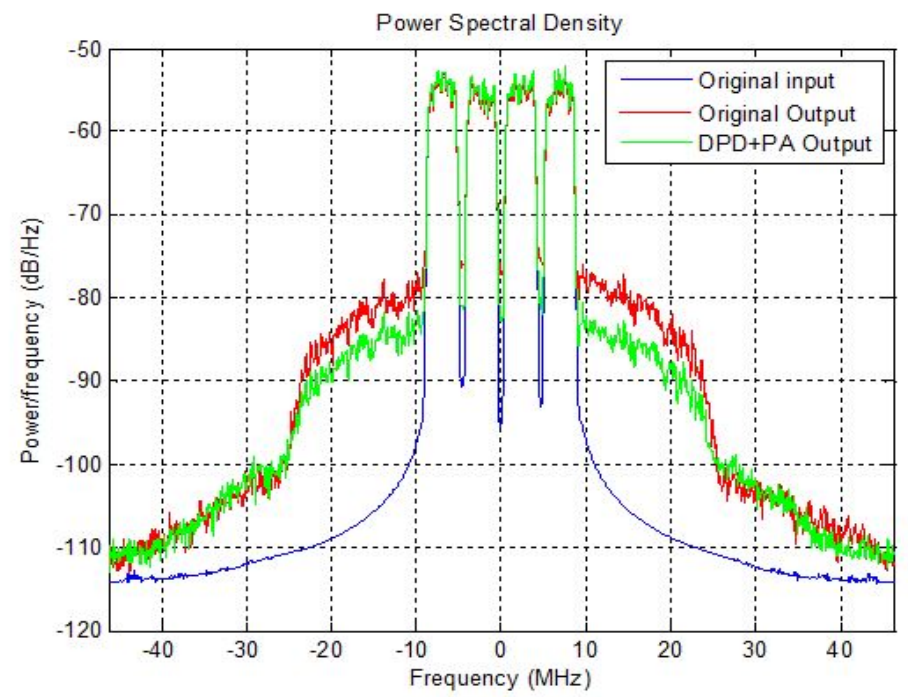

Figure 5.18: PSD plots showing linearization performance of the first order linear model ADPD for a high powered 4C-WCDMA signal.

\subsection{Memory Polynomial Based Adaptive DPD Simulation}

An adaptive DPD is simulated for the same scenerio presented in Section 5.5.2. The algorithm of this DPD requires a fixed model configuration setting for different input signals. The model configuration chosen should be able to compensate for the nonlinearity imposed on the most complex signal (signal with highest power level and 
bandwidth size) experienced by the PA.

The parameters of the model are re-estimated using an iterative procedure until they converge to provide an acceptable linearization performance. The parameters are initialized with the estimated values of the previous iteration. The time period before convergence is affected by large transient errors. Poor DPD performance is observed during these large transient errors. The transient errors during changes from a high to a low power signal can be larger than when a small change in the input power occurs.

The adaptive DPD is simulated using the memory polynomial model. This polynomial is estimated using the conventional LMS and RLS algorithms. The model structure configuration is set as $K=5, M=3$. Figure 5.19 shows the performance and transient response of an LMS based ADPD for a $-10.5 \mathrm{dBm}$ 4C-WCDMA input signal changing to a $-18.5 \mathrm{dBm}$ 2C-WCDMA signal.

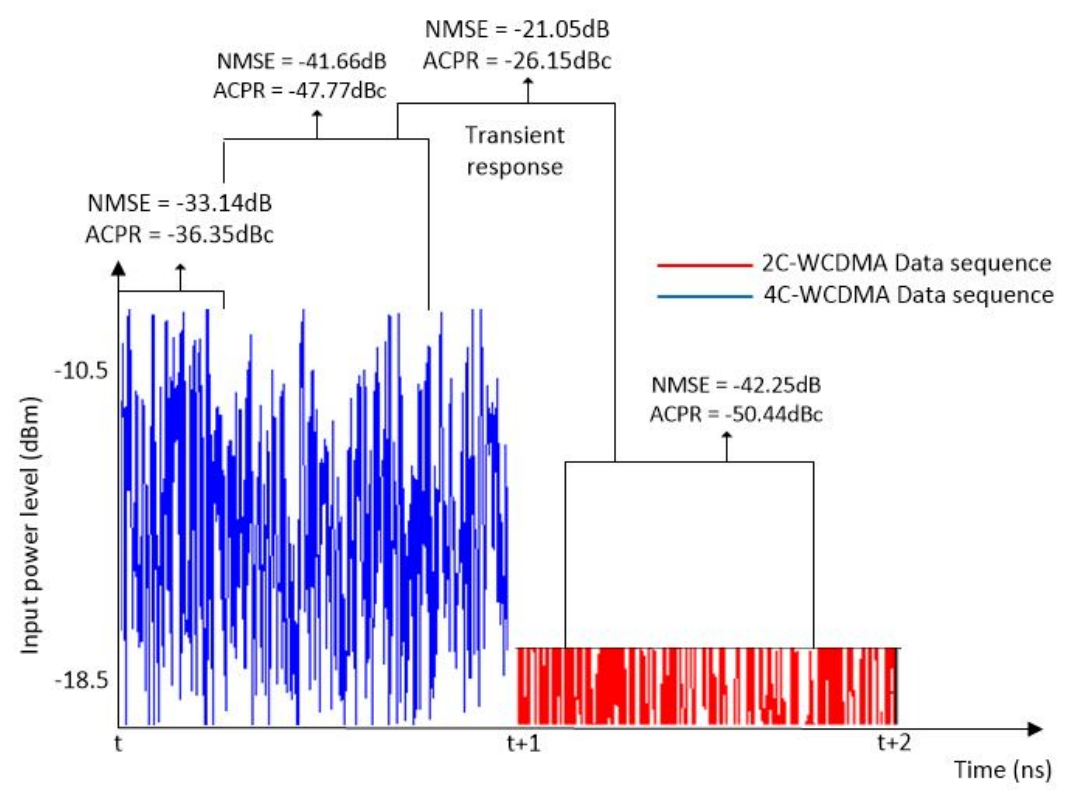

Figure 5.19: Transient response of an LMS based adaptive DPD for input signal changes with respect to time 
The results of the NMSE and ACPR show that degradation of the DPD performance occurs at the time when the input signal changes. The linearization performance cannot be maintained at acceptable values at such times. The parameters of the DPD change and only re-converge to accurately compensate for the PA distortions after an adequate number of iterations have occured from a window containing only samples from the current dataset. Considering the sequence of the datasets studied, the LMS based adaptive DPD required about 50,000 samples from the current dataset to achieve acceptable linearization performance. The RLS based adaptive DPD required about 500 samples. Table 5.9 summarizes the linearization performance of the LMS and RLS based adaptation for input signal changes of samples taken from a clear set of a particular data.

Table 5.9: Comparison of the linearization performance (NMSE \& ACPR) between the LMS ( > 50,000 samples) and RLS ( > 500 samples) based adaptive DPD

\begin{tabular}{|c|c|c||c|c|}
\hline \multirow{2}{*}{ Dataset sequence } & \multicolumn{2}{|c|}{ NMSE $(\mathrm{dB})$} & \multicolumn{2}{c|}{ ACPR $(\mathrm{dBc})$} \\
\cline { 2 - 5 } & LMS & RLS & LMS & RLS \\
\hline 4C-WCDMA @ -10.5dBm & -32.82 & -39.99 & -36.49 & -45.45 \\
2C-WCDMA @ -17.5dBm & -36.21 & -56.40 & -49.72 & -60.02 \\
2C-WCDMA @ -10.5dBm & -31.21 & -43.21 & -42.77 & -52.38 \\
2C-WCDMA @ -11.5dBm & -32.88 & -42.96 & -43.32 & -51.14 \\
4C-WCDMA @ -18.5dBm & -40.35 & -55.17 & -48.56 & -53.55 \\
2C-WCDMA @ -15.5dBm & -47.71 & -59.52 & -58.72 & -62.11 \\
2C-WCDMA @ -15.5dBm & -47.71 & -59.52 & -58.72 & -62.11 \\
2C-WCDMA @ -14.5dBm & -36.85 & -54.06 & -55.45 & -60.87 \\
2C-WCDMA @ -10.5dBm & -30.98 & -42.94 & -42.09 & -51.55 \\
4C-WCDMA @ -10.5dBm & -26.99 & -35.68 & -35.40 & -40.85 \\
\hline
\end{tabular}


The sequence of signal changes follows a pre-determined pattern that is representative of rapid signal variations in practice. Figure 5.20 shows the PSD plot of the linearization performance for an LMS and RLS based adaptive scheme for 500 samples of the two- and four-carrier WCDMA signal.

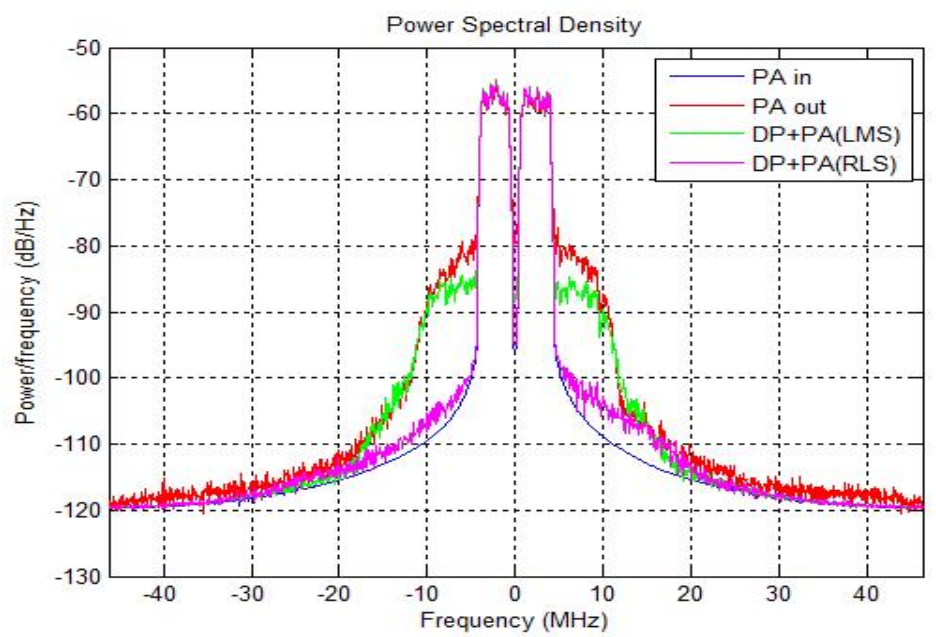

(a)

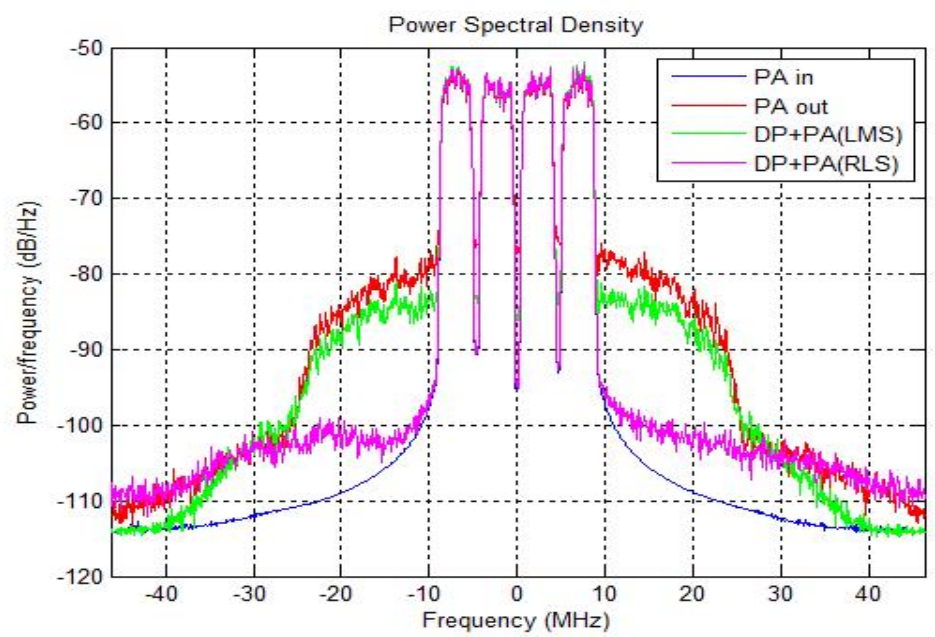

(b)

Figure 5.20: PSD plots of the original input, the original output before DPD, the simulated LMS based DPD+PA output and the simulated RLS based DPD+PA output with 1000 samples from a pure batch of high powered (a) two-carrier WCDMA signal (b) four-carrier WCDMA signal 
The result demonstrate a distortion correction of about $20 \mathrm{~dB}$ and $7 \mathrm{~dB}$ for the RLS based ADPD and LMS based ADPD respectively. An adaptive DPD with the RLS algorithm compensates for the distortions faster than one with the LMS algorithm. The batch of samples utilized to test the DPD is different from the batch of samples utilized to train the adaptive DPD.

\subsection{Multiple Model Switching Algorithm}

The linearization performance of the multiple model (MM) scheme depends on the ability of the switching algorithm to identify changes in datasets quickly and accurately. In the multiple model simulation, offline learning of the model parameters for different datasets is done to initialize the fixed $D P M_{i}$ models, the hypothesis algorithm is implemented to decide switching, and the DPD model parameters associated with the particular operating condition is selected and used to initialize the adaptive model. The adaptive model ADPM tunes the parameters to fit the particular dataset and can be used to reset the fixed $D P M_{i}$ models. The order of changes in the PA excitation signal is determined before simulation to enable examination of

- changes in signal power levels at a constant bandwidth

- changes in signal power levels with varying bandwidth

- changes in bandwidth sizes at a constant power level

- constant excitation signals

The hypothesis test based switching algorithm is applied to the input and output datasets to determine bandwidth changes and power level changes respectively. The double check ensures that wrong decisions to switch are avoided as a switch is initiated 
if either of the two tests reject the null hypothesis (signal statistics remain the same). With this, the accuracy of the decision to switch can be maintained. Table 5.10 lists the order of the incoming sequence for the datasets and the switching decision made after applying the hypothesis test to the datasets. A sample size of $N_{\text {hyp }}=500$ is adequate to ensure a $95 \%$ confidence level.

Table 5.10: Results of the hypothesis test based switching. 0 indicates that the signal's bandwidth or power level remained constant and a 1 indicates changes in the signal's characteristics

\begin{tabular}{c|c|c|c}
\hline Dataset sequence & Bandwidth test & Power level test & Switching decision \\
\hline \hline 4C-WCDMA @-10.5dBm & 0 & 0 & Start-up \\
2C-WCDMA @-17.5dBm & 1 & 1 & Yes \\
2C-WCDMA @-10.5dBm & 0 & 1 & Yes \\
2C-WCDMA @-11.5dBm & 0 & 1 & Yes \\
4C-WCDMA @-18.5dBm & 1 & 1 & Yes \\
2C-WCDMA @-15.5dBm & 1 & 1 & Yes \\
2C-WCDMA @-15.5dBm & 0 & 0 & No \\
2C-WCDMA @-14.5dBm & 0 & 1 & Yes \\
2C-WCDMA @-10.5dBm & 0 & 1 & Yes \\
4C-WCDMA @-10.5dBm & 1 & 0 & Yes \\
\hline
\end{tabular}

\subsection{Uniform Configuration Multiple Model Per- formance}

The parameters of the PA model for each dataset $D_{i}$ at an ith power level are extracted. The sample size $N_{P A}=1000$, and the uniform model configuration are $K_{P A}=5$ and $M_{P A}=3$. The LS algorithm is applied to estimate the initial parameters for each of the models $\left(D P M_{i}\right)$ in the pre-distorter. The sample size $N_{D P}=500$, and $D P M_{i}$ configurations are $K_{D P}=5$, and $M_{D P}=3$. Figures 5.21 and 5.22 show the linearization performance of the multiple model DPD in comparison with the 
LMS based adaptive DPD for low and high powered signals.

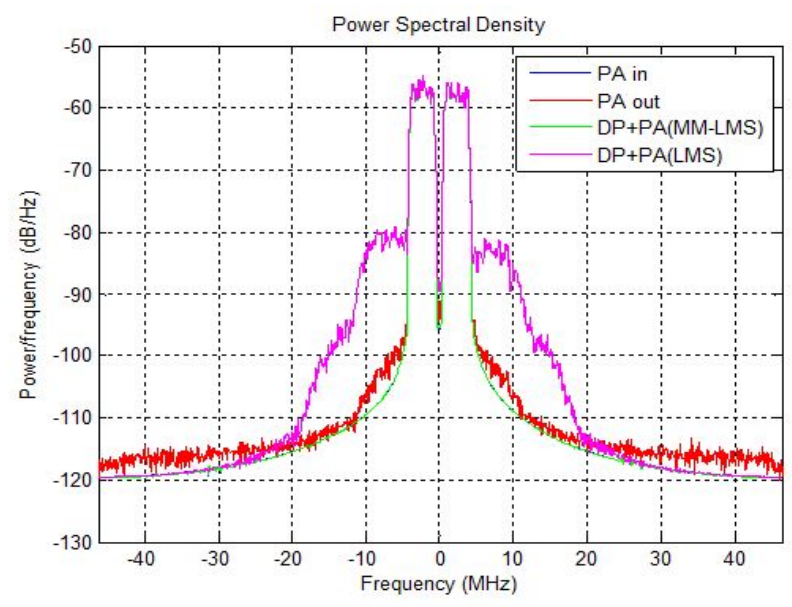

(a)

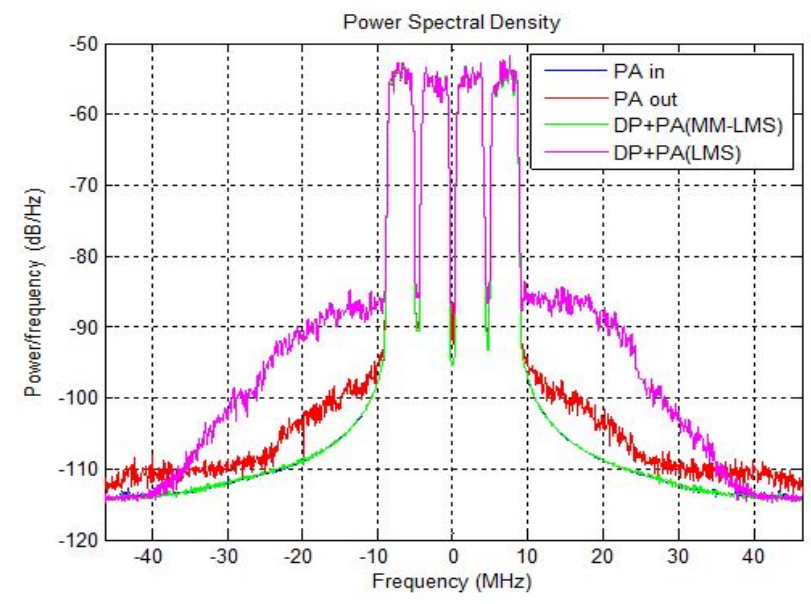

(b)

Figure 5.21: Comparison of the linearization performance of the multiple model and LMS based ADPD for low powered (a) two-carrier WCDMA signal and (b) four-carrier WCDMA signal.

When switching is initiated because of a signal change, a set of fixed parameters learned offline and stored in memory that can maintain acceptable performance is selected. The adaptive model block is initialized at the selected parameters chosen from the fixed $D P M_{i}$ model. The parameters re-converge to fit the particular datasets 
using 100 samples. However, when is not initiated, the adaptive algorithm continues with the previously estimated parameters. The estimated parameters derived after adaptation to a specific dataset can be used to reset the DPD parameters determined during offline learning.

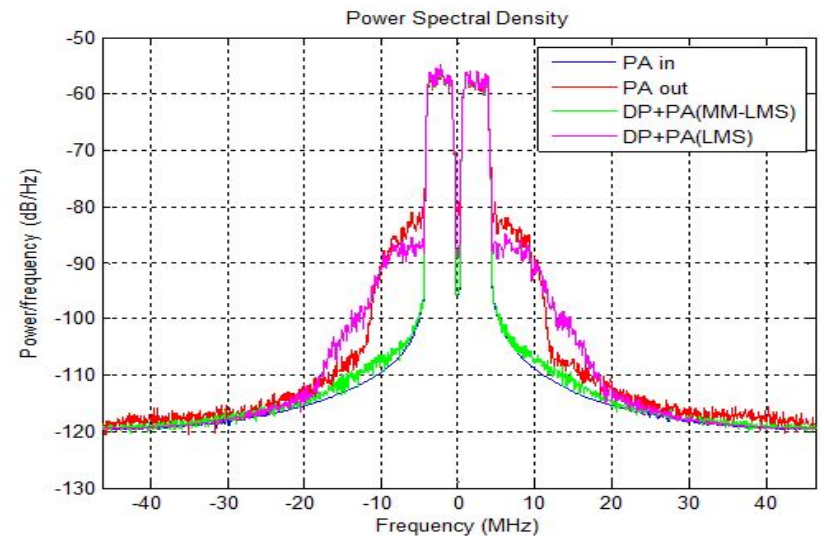

(a)

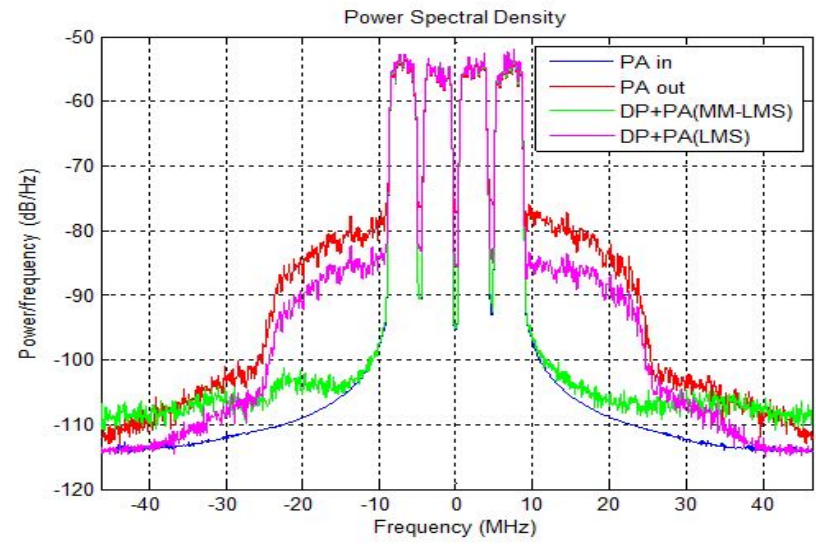

(b)

Figure 5.22: Comparison of the linearization performance of the multiple model and LMS based ADPD for high powered (a) two-carrier WCDMA signal and (b) four-carrier WCDMA signal.

The red trace of Figures 5.21(a) and (b) show the measured output of low powered 2C- and 4C-WCDMA signals. The spectrum outgrowth worsens with an LMS based 
ADPD (pink trace) compared to the distortion reduction provided by the multiple model scheme (green trace). The spectrum regrowth cannot be reduced with the LMS based ADPD and is observed to worsen in the case of low powered signals. The distortion reduction of the LMS based ADPD for high powered signals is significantly far lesser than that of the proposed scheme as shown in Figures 5.22(a) and (b). The results indicate that the MM scheme achieves and maintains acceptable linearization performance for input signal changes at different power levels.

The linearization performance of the RLS based ADPD is also compared to the MM scheme. As demonstrated from the results of Section 5.7, the RLS based ADPD can achieve acceptable performance with 500 samples from a window of a particular dataset at an ith power level. However, it is not certain that the performance can be maintained for all signal changes. This is demonstrated to be true from the NMSE and ACPR results for the RLS based ADPD and MM scheme using the LMS algorithm (MM-LMS) listed in Table 5.10. The RLS based ADPD is not guaranteed to maintain

Table 5.11: Comparison of the linearization performance (NMSE \& ACPR) of the RLS based ADPD and the multiple model scheme at varying signal changes

\begin{tabular}{c|c|c|c}
\hline \hline Dataset selected & Algorithms & ACPR $(\mathrm{dBc})$ & NMSE $(\mathrm{dB})$ \\
\hline \multirow{3}{*}{ 2C-WCDMA @-17.5dBm } & Without DPD & -54.75 & - \\
\cline { 2 - 4 } & RLS & -60.89 & -51.49 \\
\cline { 2 - 4 } & MM-LMS & -62.73 & -61.48 \\
\hline \hline \multirow{3}{*}{ C-WCDMA @-10.5dBm } & Without DPD & -36.07 & - \\
\cline { 2 - 4 } & RLS & -48.90 & -35.78 \\
\cline { 2 - 4 } & MM-LMS & -51.05 & -38.50 \\
\hline \hline \multirow{3}{*}{$4 \mathrm{C}-W C D M A @-18.5 \mathrm{dBm}$} & Without DPD & -46.57 & - \\
\cline { 2 - 4 } & RLS & -52.13 & -50.03 \\
\cline { 2 - 4 } & MM-LMS & -53.51 & -55.03 \\
\hline \hline \multirow{3}{*}{ 4C-WCDMA @-10.5dBm } & Without DPD & -23.06 & - \\
\cline { 2 - 4 } & RLS & -39.84 & -32.76 \\
\cline { 2 - 4 } & MM-LMS & -46.43 & -35.69 \\
\hline
\end{tabular}


the performance for all signal changes. The ACPR and NMSE values are shown to increase significantly above the $-45 \mathrm{dBc}$ and $-35 \mathrm{~dB}$ constraints respectively with the RLS based ADPD for a $-18.5 \mathrm{dBm}$ 4C-WCDMA signal changing to a $-10.5 \mathrm{dBm} 4 \mathrm{C}$ WCDMA signal. The MM-LMS is seen to maintain linearization performance at the time of this change.

Furthermore, the linearization performance of the proposed multiple model approach is assessed in comparison with the RLS based ADPD and LMS based ADPD Figures 5.23 and 5.24 show the PSD plots for the output signal: without a DPD, with the RLS based ADPD, with the LMS based ADPD, and the proposed multiple model scheme The plots show that the proposed scheme gives the highest spectral reduction (green traces) followed by the black traces representing the output spectra for the RLS based ADPD. The LMS based ADPD gives the least spectral reduction as shown by the pink traces.

\subsection{Discussion}

Overall, the results of Section 5.9 are discussed here.

\section{Linearization Performance Results}

The simulation results of Section 5.9 show that the LMS based ADPD gives a poor performance that violates the constraints on the ACPR and NMSE values as the PA excitation signal changes. The linearization performance deteriorates at the point of a sudden change and a good linearization performance can only be guaranteed after 50000 samples from a window containing only samples from a particular dataset have been processed. In other words, more than 50000 samples have to be processed before acceptable requirements are met. In a situation where 50000 samples from a 


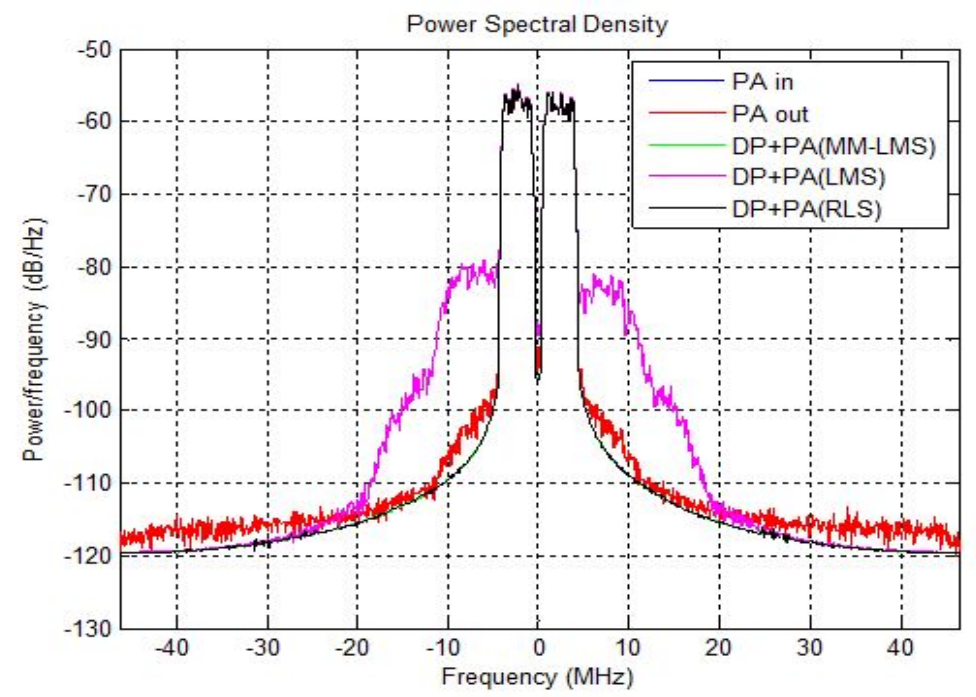

(a)

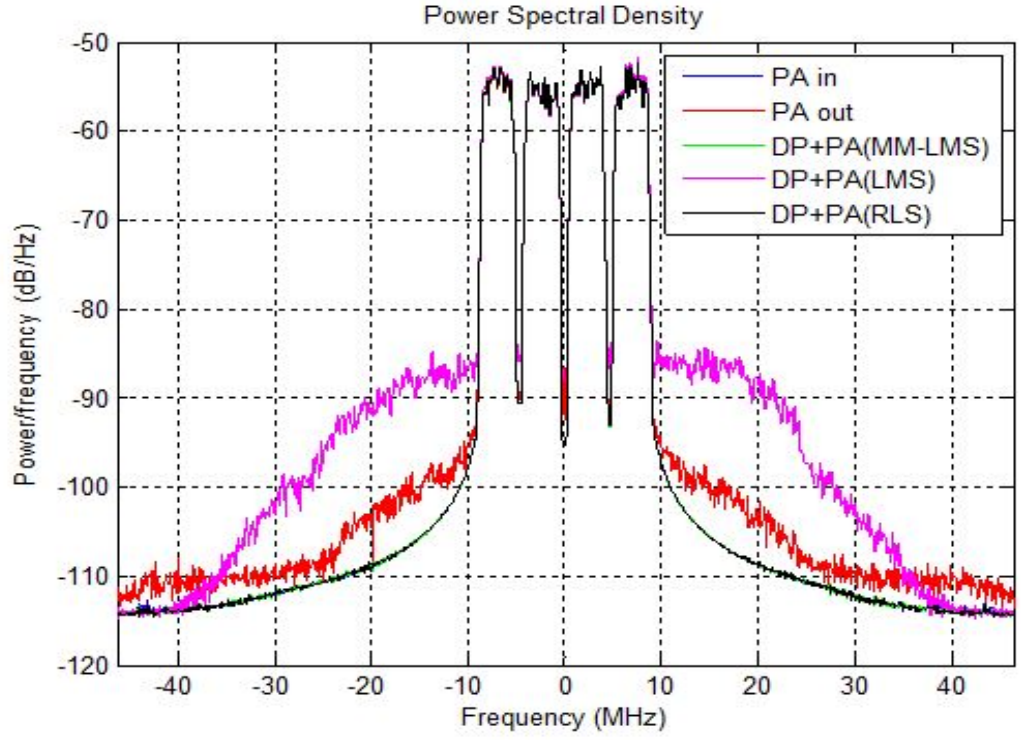

(b)

Figure 5.23: Measured PA output spectra showing: the measured input (blue), measured output without a DPD (red), output with the LMS based adaptive pre-distorter (pink), RLS based adaptive pre-distorter (black), and LMS based multiple model (green) for signal change to (a) -17.5dBm two-carrier WCDMA signal, (b) -17.5dBm four-carrier WCDMA signal. 


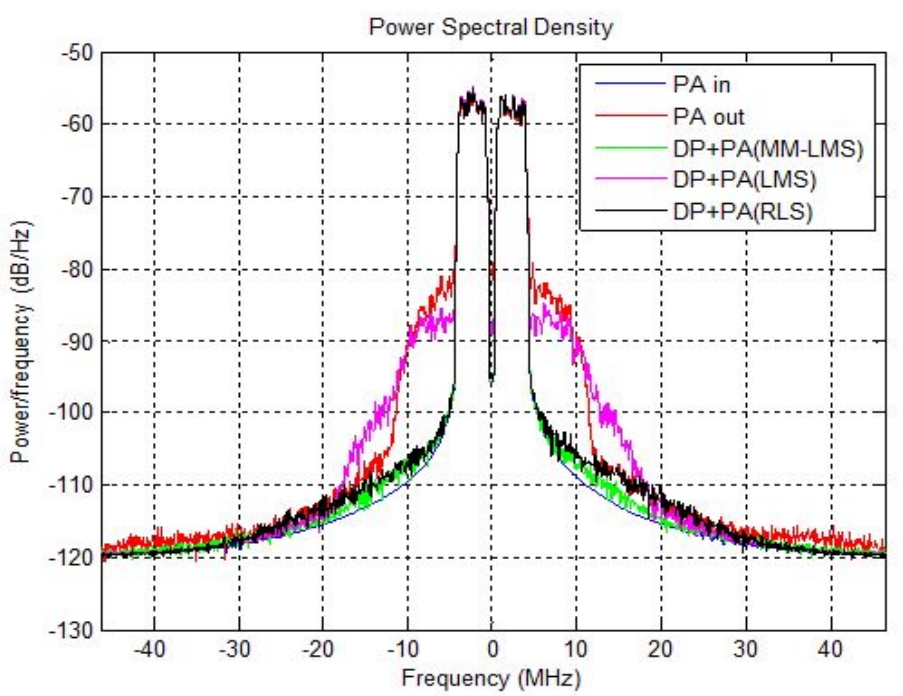

(a)

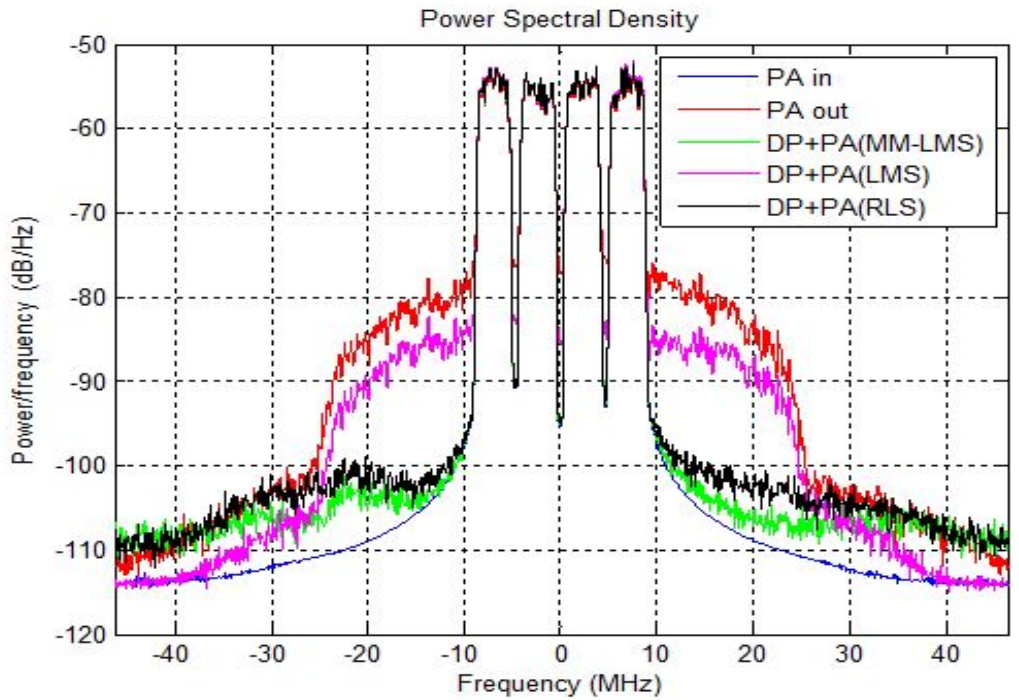

(b)

Figure 5.24: Measured PA output spectra showing: the measured input (blue), measured output without a DPD (red), output with the LMS based adaptive ADPD (pink), RLS based adaptive ADPD (black), and proposed multiple model scheme (green) for (a) -10.5dBm two-carrier WCDMA signal, (b) -10.5dBm four-carrier WCDMA signal. 
particular dataset at ith power level cannot be processed because signals change even more rapidly, the DPD performance degrades even more and becomes unstable.

The plots show that the RLS based ADPD with 500 samples can achieve comparable performance as the proposed scheme. However, this level of performance cannot be maintained when signals switch from a high powered signal to a low powered signal. When a signal switches from a high power (-10.5dBm 2C-WCDMA signal) to a low power (-18.5dBm 4C-WCDMA signal ) as shown in Table 5.11, the DPD has been trained to compensate for the PA over the entire dynamic range and can still work properly with the PA excited by the lower signal. For a low to a high power signal switch, the parameters of the DPD are not sufficient to compensate for the higher nonlinearity experienced. A switch from the $-18.5 \mathrm{dBm}$ 4C-WCDMA signal to the $-10.5 \mathrm{dBm}$ 4C-WCDMA in Table 5.11 shows the deterioration in the performance of the RLS based ADPD.

The MM scheme proposed maintains linearization performance for all switching possiblities. This is as a result of the offline-learned parameters used to initialize the adaptation for signal changes. The hypothesis test based switching technique can accurately deal with the problem of switching with 500 samples. This makes it faster and more suitable for use in a rapidly changing PA input signal scenerio.

\section{Complexity and Speed}

The LMS based ADPD computation per sample requires $K \times M$ complex multiplications which is equal to $4 \times K \times M$ real multiplications. The computation of the LMS based ADPD goes through 50000 samples to achieve acceptable performance. Thus, $4 \times 50000 \times\{K \times M\}^{2}$ real multiplications is required for every signal change. However, the RLS based ADPD attaining good performance with 500 sam-

ples will be computed using $500 \times\{K \times M\}^{2}$ complex multiplication equivalent to $4 \times 500 \times\{K \times M\}^{2}$ real multiplications. 
The hypothesis test algorithm in the MM scheme involves no matrix inversions or complex multiplications. The computation involves additions, subtractions and divisions operating on 500 samples to monitor signal changes. The adaptation of parameters to new values requires 100 samples during signal changes. Computation

will require $4 \times 100 \times\{K \times M\}$ real multiplications. The memory storage for the initial parameters estimated is not considered. It is assumed that this will have little or no significant effect on the computational complexity. The one-time offline computation of the initial parameters is also not considered.

The comparison of the number of samples and computations required by each scheme indicates that the MM scheme offers a faster adaptation with reduced complexity compared to other schemes discussed here.

\subsection{Non-uniform Configuration Multiple Model Performance}

The sets of models in the multiple model DPD scheme can be configured with different nonlinear and memory orders depending on the power level of the input signal. The results in Section 5.5 have shown that low DPD model configurations are adequate when the PA experiences low powered signals while high powered signals require higher configurations. The non-uniform configuration multiple model scheme uses this idea to reduce the number of parameters to be estimated and the computation complexity associated with the estimation when signal changes to a low powered signal. Table 5.12 shows the choice of configurations for the particular datasets used in this thesis. At initialization, the number of parameters to be estimated is reduced. Table 5.13 shows the parameter savings at initialization for the non-uniform multiple 
Table 5.12: Choice of model configuration settings chosen for different power level ranges

\begin{tabular}{l|c|c}
\hline Dataset $\left(D_{r}\right)$ & Power level range & Model configuration \\
\hline $\begin{array}{l}\text { 2C-WCDMA } \\
\text { 4C-WCDMA }\end{array}$ & $-10.5 \mathrm{dBm}$ to $-14.5 \mathrm{dBm}$ & $K=5, M=3$ \\
\hline \hline $\begin{array}{l}\text { 2C-WCDMA } \\
\text { 4C-WCDMA }\end{array}$ & $-15.5 \mathrm{dBm}$ to $-19.5 \mathrm{dBm}$ & $K=3, M=2$ \\
\hline
\end{tabular}

model configuration compared to the uniform configuration. When the input signal changes from a high powered to a low powered signal, the number of parameters to be estimated is reduced from 15 to 6 . Figure 5.25 shows the performance of

Table 5.13: Parameter savings estimation

\begin{tabular}{c|l|c|c|c}
\hline Configuration & Power levels & Parameters/model & Models & Total \\
\hline Uniform & & 15 & 19 & 285 \\
\hline \hline \multirow{2}{*}{ Non-uniform } & High & 6 & 9 & $54+150=204$ \\
& Low & 15 & 10 & 81 \\
\hline \multicolumn{4}{c}{ Parameter savings }
\end{tabular}

the non-uniform configuration multiple model scheme using $K=3, M=2$ for a low powered signal PA excitation compared to the performance of the uniform configuration that requires $K=5, M=3$ for the same low powered signal. the plots show that the spectrum regrowth seen in the red trace representing the output without DPD is reduced to the same level by both configurations For the datasets used, the performance of the multiple model scheme with a configuration of $K=5, M=3$ for high powered signals is compared to a configuration of $K=7, M=5$. Figure 5.26 shows the comparable performance achieved using a multiple model configuration of $K=5, M=3$ and $K=7, M=5$. 


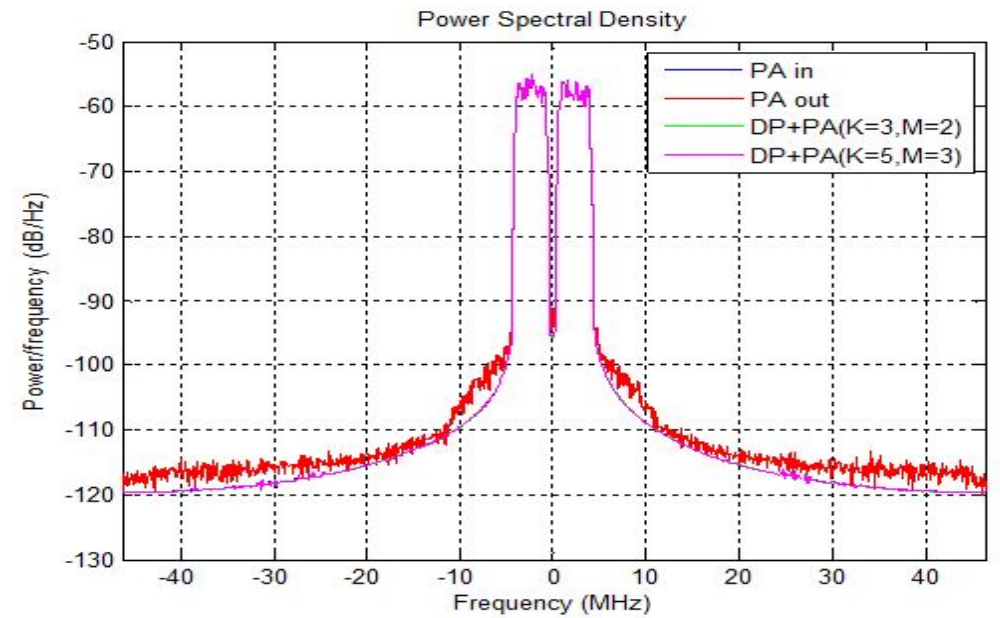

(a)

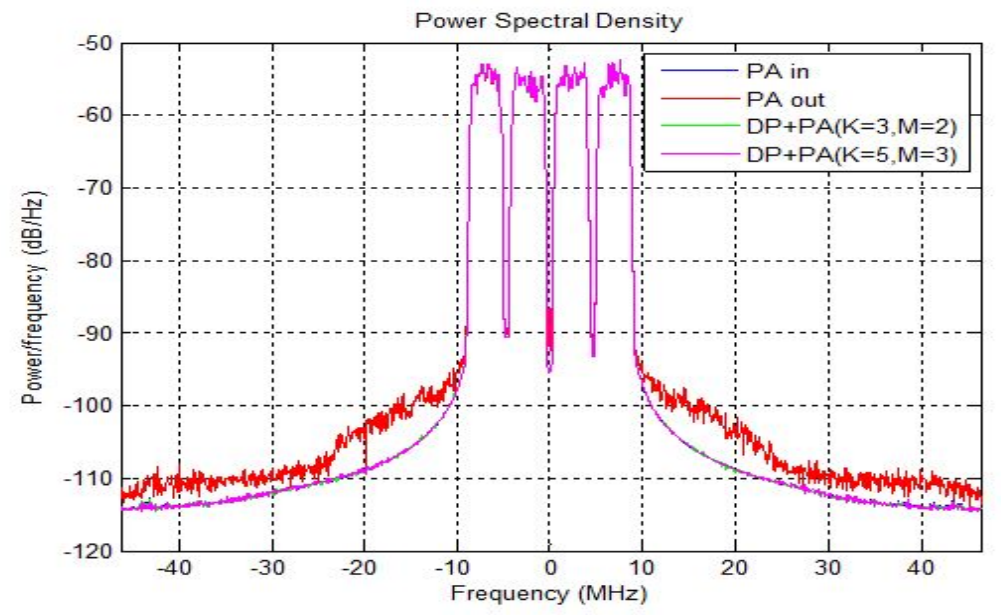

(b)

Figure 5.25: Comparison of the linearization performance of the multiple model scheme at different configurations with low powered signals for (a) 2C-WCDMA and (b) 4C-WCDMA 


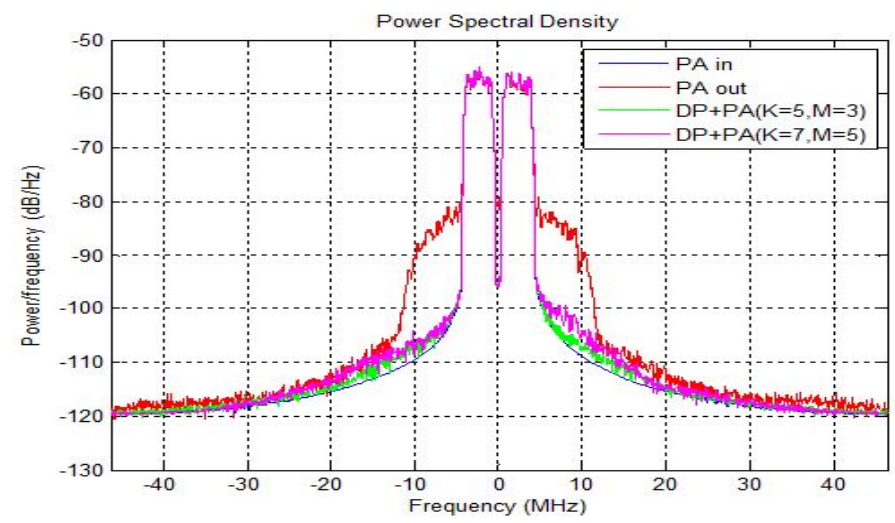

(a)

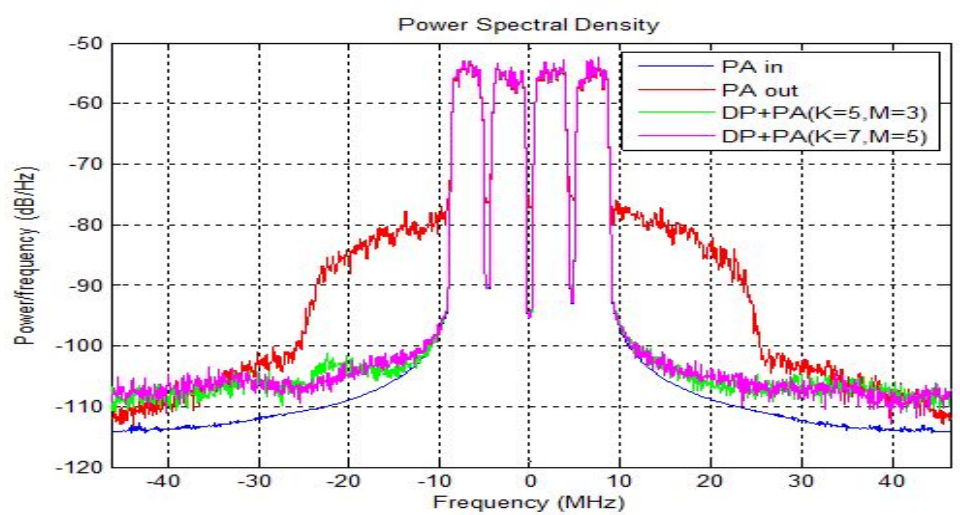

(b)

Figure 5.26: Comparison of the linearization performance of the multiple model scheme at different configurations with high powered signals for (a) 2CWCDMA and (b) 4C-WCDMA

It is observed that the model configuration of $K=5, M=3$ is adequate for the highest power signal measured from the PA. A higher configuration of $K=7, M=5$ will only increase the computation complexity without an associated improvement in the linearization performance. 


\subsection{Discussion}

The non-uniform configuration multiple model scheme is shown to further reduce the computation complexity and increase speed of the proposed DPD scheme. The offline estimation of initial parameters provides an opportunity to understand the PA before it is set to operate in the basestation. The test signals at varying power levels used for initialization are similar to the ones to be experienced in practice. The highest nonlinearity of the PA can be studied and the appropriate model configuration for each operating condition of the PA can be decided beforehand. The benefit of this is a faster adaptation when any slight reduction in the number of parameters to be estimated significantly improves the speed of the DPD.

\subsection{Online Adaptive Parameter Learning}

The re-converged parameters estimated after a switch has been initiated due to a signal change can be used to reset the offline estimated parameters of the fixed $D P M_{i}$ models of the multiple model scheme. This describes the online learning adaptive portion for the proposed scheme. At a time $\mathrm{t}$ when the PA is excited by a signal $x_{a}(n)$, the chosen fixed parameters learned offline serve as a strategic initialization point for adaptation.

When the excitation signal changes at a time $\mathrm{t}+1$ to $x_{b}(n)$, the adaptation is reinitialized with the parameters of the appropriate model related to the power level of $x_{b}(n)$. The resulting parameters after every adaptation for $x_{a}(n)$ and $x_{b}(n)$ are used to reset the fixed parameters learned offline associated with the power level of the signal. At any other time $\mathrm{t}+\mathrm{q}$ when either $x_{a}(n)$ or $x_{b}(n)$ re-excite the PA, the learned parameters stored are selected. The excitation of the PA by re-occurring signals will require little or no adaptation with this resetting capability of the adaptive model in 
the proposed scheme.

Adaptation from the online learned parameters only provides slight improvement to the linearization performance and it is can be possible to cut-off the adaptive scheme after all possible operating conditions of the PA have been learned over time. Table 5.14 shows the linearization performance for the first and subsequent occurrences of the $2 \mathrm{C}$ - and $4 \mathrm{C}$-WCDMA signal as the PA excitation signal at different times. It may be true with the data used for simulation that after learning has ocTable 5.14: NMSE and ACPR for 2C- and 4C-WCDMA signals exciting the PA at time $\mathrm{t}$ (first occurrence) and at time $\mathrm{t}+\mathrm{q}$ (subsequent occurence) when offline or online learned parameters are used

\begin{tabular}{|c|c|c|c|c|c|}
\hline \multirow{2}{*}{ Time } & \multirow{2}{*}{ Parameters } & \multicolumn{4}{|c|}{ Signal } \\
\cline { 3 - 6 } & & \multicolumn{2}{|c|}{ 2C-WCDMA } & \multicolumn{2}{|c|}{ 4C-WCDMA } \\
\cline { 3 - 6 } & & NMSE (dB) & ACPR (dBc) & NMSE (dB) & ACPR (dBc) \\
\hline \multirow{2}{*}{$\mathrm{t}$} & Offline & -38.50 & -51.05 & -35.69 & -46.43 \\
\hline \multirow{2}{*}{$\mathrm{t}+\mathrm{q}$} & Offline & -38.50 & -51.05 & -35.69 & -46.43 \\
\cline { 2 - 6 } & Online & -39.57 & -51.08 & -36.71 & -46.47 \\
\hline
\end{tabular}

cured for all operating conditions, no further changes happen to the parameters and consequently no further improvement in the linearization performance. Therefore, adaptation may seem no longer necessary as the PA model is only described at these levels. However, it is worth noting that factors affecting the operating conditions of the PA can also be long-term [42] and different from the short-term factors (varying input signal characteristics) considered in this work.

The consideration of other factors beyond the scope of the work in this thesis that can still affect the linearization performance of the DPD is an important issue in a real DPD deployment. In such cases, adaptation from learned parameters will remain necessary and desired. A more advanced learning module will be to store parameters into newly generated $D P M_{i}$ models for different operating conditions encountered during the course of learning. 


\subsection{Number of Models Reduction}

This thesis focused on the impact of varying input signals on the PA behavior. The plots of the input and output signals extracted from a PA at different power levels confirm that the PA behavior for each dataset at a particular bandwidth size and power level is different. A certain dataset cannot be used to model the PA for the full dynamic range of operation of the PA. In turn, the DP exhibits different characteristics with respect to different operating conditions of the PA. In simulation, unique parameters of the PA model are estimated for each dataset to be used in the multiple model scheme.

In order to reduce the number of models in the multiple model scheme, the power range of low powered signals can be increased. In this thesis, three low powered datasets at $-17.5 \mathrm{dBm}$ to $-19.5 \mathrm{dBm}$ are combined to represent all signals at a power level $<-17.5 \mathrm{dBm}$. The parameters of each model at these power levels can be combined through coefficient averaging to give only one set of model parameters.

The first order linear model can also be used in place of low configuration MP models for low powered input signals. Further reduction of complexity and improved speed can be gained by incorporating the simpler linear model at low power level ranges. However, the smaller ranges considered for high powered signals is still required because nonlinear behavior varies significantly from one another with small power variations for high powered signals.

\subsection{Summary}

This chapter presented the simulation results from the test and validation of the proposed multiple model scheme. Real data signals of a PA were obtained from an 
experimental platform and utilized to model the digital pre-distorter using the MATLAB simulation tool. The PA linearisation performance, complexity and response time of the digital pre-distorter was studied using input signals with different bandwidth sizes. The simulation results of the static and adaptive pre-distorter schemes was used for comparison with the proposed scheme to demonstrate its effectiveness.

It was observed that the LMS based ADPD offered large transient errors and instability in performance. The RLS based ADPD offered faster convergence of DPD parameters but at the cost of high computation complexity. The multiple model scheme was shown to offer faster adaptation to maintain the linearization performance for all possible signal changes with reduced computation complexity. The hypothesis test based switching incorporated with the proposed scheme used 500 samples for very fast switching. 


\section{Chapter 6}

\section{Conclusion and Future Work}

\subsection{Conclusion}

The work presented in this thesis establishes the importance of an adaptive DPD scheme as a solution to rapidly changing PA behavior caused by the variations in the input excitation signals. A static DPD designed to compensate for the nonlinearity at the operating condition of a PA can adequately linearize the PA. The current operating condition is defined by the input signal at a particular power level and bandwidth. However, the performance of the DPD at all other times when input signal changes have occurred is undesirable.

The static DPD scheme is also associated with significantly high computation complexity. A new batch of samples from the current dataset have to be collected and the parameters re-estimated. Thus, they can only be desired for PAs that are not expected to change behaviors over a long period of time. For such PAs, it is assumed that the input signals do not change and only slow-varying long-term factors such as temperature can affect the PA during its operation.

Adaptive DPD schemes offer real time online compensation that can continuously adapt to the PA's changing behavior. However, one major problem with the 
conventional schemes is that the linearization performance cannot be maintained at acceptable values. The estimators used in the adaptive schemes have computational complexity problems. Rapid fluctuations in power levels and bandwidth sizes degrade the PA performance and the adaptive DPD requires more time to track the rapid fluctuations that may result in relatively large transient errors.

Furthermore, adaptive schemes require the use of a single fixed model and configuration for estimating the parameters of the DPD. The configuration is set to suit the most complex signal to be experienced by the PA and this remains constant for all power levels and bandwidth sizes of the input signal. Therefore, the same number of parameters are required to be estimated for all power levels. Low power operating conditions that can be sufficiently represented with fewer parameters are overparameterized.

The multiple model adaptive DPD is explored in this work as a solution to increase convergence speed and reduce computation complexity of achieving digital pre-distortion. The structure of the multiple model scheme consists of a hybrid of the static and adaptive estimators, and it incorporates the hypothesis switching algorithm to accurately decide when to switch. To demonstrate the viability of the multiple model scheme with a real PA, real WCDMA test signals were used in simulations.

Simulations showed that the multiple model adaptive DPD scheme was able to maintain the linearization performance at an acceptable value for all possible input signal variations defined by standard set NMSE and ACPR thresholds. The scheme also offered faster adaptation in terms of the number of signals required to be processed per signal change. The RLS based adaptive DPD offered relatively fast re-convergence speed at signal changes. However, the RLS based ADPD cannot maintain a good performance for all situations. Furthermore, the multiple model scheme 
offered reduced computation complexity than the RLS based ADPD.

In general, the multiple model scheme yielded lower transient error response than the conventional adaptive schemes. In comparison with other existing work that propose variants of the conventional adaptive schemes, the proposed scheme was simulated/demonstrated for a PA operating over a wide dynamic range within a very short period of time. In addition, the reduced cost implication of the proposed scheme is highly significant. The reduced computation complexity means reduced resources in hardware implementations. Linearization for low powered signals can be achieved using fewer parameters derived from estimation.

Part of the work done in this thesis also investigated the need for using different sets of parameters to model the PA for signals at different power levels. From simulations, it was seen that a set of parameters cannot be used to represent the PA behavior in its full dynamic range. Although a wide range in low operating power conditions can be represented with a set of model parameters, the same cannot be applied when the PA is operating at higher nonlinear modes. Moreover, the PA behavior of adjacent higher power operating regions are significantly different from each other.

\subsection{Future Work}

The work presented in this thesis provides potential directions for future research. The viability of the proposed scheme demonstrated through simulation can be extended by implementing the scheme in real hardware to test the performance and validate the idea proposed in the scheme.

One of the advantages of the multiple model scheme is the flexibility in the choice of the model structure, configuration, and estimation algorithm for the set of models 
used in the DPD. The set-up used in this thesis explored using only non-uniform configurations in the models. Hence, more work to explore the possibilities of using varying model structures and estimation algorithms can be done. Studying the effect of these other possibilities on the overall performance of the DPD will be an interesting area to investigate.

The PA in the equipment testbed platform for acquiring measurement data used in this work exhibits weak nonlinearity that can sufficiently be modeled with 15 parameters. Current PAs have severely high nonlinearities that would require more than fifteen parameters to generate an unbiased estimate of the PA and in effect, improve the efficiency of the DPD.

The power levels and amplitude settings considered in this work were nonoverlapping. It would be an area of interest to explore results for overlapping power level ranges and power levels that fall in between the ones considered in this work. Moreover, the reduction of the number of models required in the DPD, explored by increasing the range for the amplitude setting and power levels of the input signal, can also be considered at overlapping levels.

The adaptive learning component of the proposed scheme can further be researched to improve the effectiveness and reduce the overall complexity of the proposed scheme. Low powered signals can be modeled with a fixed set of parameters that will not be reset by the adaptive scheme. The adaptive learning component can be designed to function only with the higher power level signals that require more adaptation to achieve better linearization performance. 


\section{References}

[1] F. Mkadem, Behavioural Modeling and Linearization of RF Power Amplifier using Artificial Neural Networks. PhD thesis, University of Waterloo, May 2010.

[2] Chih-Hung Lin, Hsin-Hung Chen, Yung-Yi Wang, and Jiunn-Tsair Chen, "Dynamically optimum lookup-table spacing for power amplifier predistortion linearization," IEEE Transactions on Microwave Theory and Techniques, vol. 54, pp. 2118-2127, May 2006.

[3] A. Wright and W. Durtler, "Experimental performance of an adaptive digital linearized power amplifier (for cellular telephony)," IEEE Transactions on Vehicular Technology, vol. 41, no. 4, pp. 395-400, 1992.

[4] X. Yu, Contributions to Digital Predistortion of Radio-Frequency Power Amplifiers for Wireless Applications. PhD thesis, Contributions to Digital Predistortion of Radio-Frequency Power Amplifiers for Wireless Applications, 2012.

[5] O. Andersen, P. Malmlof, and D. Wisell, "Nonlinear Characterization of Multiple Carrier Power Amplifiers," in 56th ARFTG Conference Digest, vol. 38, pp. 1-8, IEEE, Nov. 2000.

[6] F. Luo, Digital Front-End in Wireless Communications and Broadcasting: Circuits and Signal Processing. Cambridge University Press, 2011.

[7] A. S. Tehrani, T. Eriksson, and C. Fager, "Modeling of long term memory effects in RF power amplifiers with dynamic parameters," in 2012 IEEE/MTT-S International Microwave Symposium Digest, pp. 1-3, IEEE, June 2012.

[8] F. Ghannouchi and O. Hammi, "Behavioral modeling and predistortion," IEEE Microwave Magazine, vol. 10, pp. 52-64, Dec. 2009.

[9] S. Andreoli, H. McClure, P. Banelli, and S. Cacopardi, "Digital linearizer for RF amplifiers," IEEE Transactions on Broadcasting, vol. 43, pp. 12-19, Mar. 1997. 
[10] C. Potter, "System analysis of a W-CDMA base-station PA employing adaptive digital predistortion," IEEE Radio Frequency Integrated Circuits (RFIC) Symposium., 2002.

[11] A. D'Andrea, V. Lottici, and R. Reggiannini, "A digital approach to efficient RF power amplifier linearization," in GLOBECOM 9\%. IEEE Global Telecommunications Conference. Conference Record, vol. 1, pp. 77-81, IEEE, 1997.

[12] S. A. Bassam, M. Helaoui, and F. M. Ghannouchi, "2-D Digital Predistortion (2-D-DPD) Architecture for Concurrent Dual-Band Transmitters," IEEE Transactions on Microwave Theory and Techniques, vol. 59, pp. 2547-2553, Oct. 2011.

[13] D. Morgan, Z. Ma, J. Kim, M. Zierdt, and J. Pastalan, "A Generalized Memory Polynomial Model for Digital Predistortion of RF Power Amplifiers," IEEE Transactions on Signal Processing, vol. 54, pp. 3852-3860, Oct. 2006.

[14] L. Ding, G. Zhou, D. Morgan, Z. Ma, J. Kenney, J. Kim, and C. Giardina, "A Robust Digital Baseband Predistorter Constructed Using Memory Polynomials," IEEE Transactions on Communications, vol. 52, pp. 159-165, Jan. 2004.

[15] J. Kim and K. Konstantinou, "Digital predistortion of wideband signals based on power amplifier model with memory," Electronics Letters, vol. 37, no. 23, p. 1417, 2001.

[16] O. Ata, "Predistort-linearisation method for future basestation amplifiers of cellular radio," in Proceedings of PIMRC '96 - rth International Symposium on Personal, Indoor, and Mobile Communications, vol. 2, pp. 267-271, IEEE, 1996.

[17] J. Cavers, "Amplifier linearization using a digital predistorter with fast adaptation and low memory requirements," IEEE Transactions on Vehicular Technology, vol. 39, no. 4, pp. 374-382, 1990.

[18] S. Boumaiza and F. M. Ghannouchi, "Realistic power-amplifiers characterization with application to baseband digital predistortion for $3 \mathrm{G}$ base stations," IEEE Transactions on Microwave Theory and Techniques, vol. 50, pp. 3016-3021, Dec. 2002.

[19] K. Lee and P. Gardner, "Comparison of different adaptation algorithms for adaptive digital predistortion based on EDGE standard," in 2001 IEEE MTT-S International Microwave Sympsoium Digest, vol. 2, pp. 1353-1356, IEEE, 2001. 
[20] T. Fujinaka and S. Omatu, "A switching scheme for adaptive control using multiple models," in IEEE SMC'99 Conference Proceedings. 1999 IEEE International Conference on Systems, Man, and Cybernetics (Cat. No.99CH37028), vol. 5, pp. 80-85, IEEE, 1999.

[21] Y. Nagata, "Linear amplification technique for digital mobile communications," in IEEE 39th Vehicular Technology Conference, pp. 159-164, IEEE, 1989.

[22] D. M. Giesbers, Adaptive digital polynomial predistortion linearisation for $R F$ power amplifiers. PhD thesis, University of Canterbury, 2008.

[23] A. D'Andrea, V. Lottici, and R. Reggiannini, "RF power amplifier linearization through amplitude and phase predistortion," IEEE Transactions on Communications, vol. 44, no. 11, pp. 1477-1484, 1996.

[24] S. C. Cripps, Advanced Techniques in RF Power Amplifier Design. Artech House, 2002 .

[25] S. Boumaiza, T. Liu, and F. Ghannouchi, "On the Wireless Transmitters Linear and Nonlionear Distortions Detection and Pre-Correction," in 2006 Canadian Conference on Electrical and Computer Engineering, pp. 1510-1513, IEEE, 2006.

[26] D. Wisell, "A baseband time domain measurement system for dynamic characterization of power amplifiers with high dynamic range over large bandwidths," in Proceedings of the 20th IEEE Instrumentation Technology Conference, vol. 2, pp. 1177-1180, IEEE, 2003.

[27] K. M. Gharaibeh, Nonlinear Distortion in Wireless Systems: Modeling and Simulation with MATLAB. John Wiley \& Sons, 2011.

[28] A. Saleh, "Frequency-Independent and Frequency-Dependent Nonlinear Models of TWT Amplifiers," IEEE Transactions on Communications, vol. 29, pp. 17151720, Nov. 1981.

[29] J. Stonick, V. Stonick, J. Moura, and R. Zborowski, "Memoryless polynomial adaptive predistortion [TV transmitters]," in 1995 International Conference on Acoustics, Speech, and Signal Processing, vol. 2, pp. 981-984, IEEE, 1995.

[30] G. Montoro, P. L. Gilabert, E. Bertran, A. Cesari, and D. D. Silveira, "A New Digital Predictive Predistorter for Behavioral Power Amplifier Linearization," IEEE Microwave and Wireless Components Letters, vol. 17, pp. 448-450, June 2007. 
[31] O. Hammi and F. Ghannouchi, "Twin Nonlinear Two-Box Models for Power Amplifiers and Transmitters Exhibiting Memory Effects With Application to Digital Predistortion," IEEE Microwave and Wireless Components Letters, vol. 19, pp. 530-532, Aug. 2009.

[32] A. Zhu, J. C. Pedro, and T. J. Brazil, "Dynamic Deviation Reduction-Based Volterra Behavioral Modeling of RF Power Amplifiers," IEEE Transactions on Microwave Theory and Techniques, vol. 54, pp. 4323-4332, Dec. 2006.

[33] L. Ding and G. Zhou, "Effects of Even-Order Nonlinear Terms on Power Amplifier Modeling and Predistortion Linearization," IEEE Transactions on Vehicular Technology, vol. 53, pp. 156-162, Jan. 2004.

[34] A. Bateman, R. Wilkinson, and J. Marvill, "The application of digital signal processing to transmitter linearisation," in 8th European Conference on Electrotechnics, Conference Proceedings on Area Communication, pp. 64-67, IEEE, 1988.

[35] Y. Jung and M. Enqvist, "Estimating models of inverse systems," in 52nd IEEE Conference on Decision and Control, pp. 7143-7148, IEEE, Dec. 2013.

[36] O. Hammi, S. Boumaiza, F. M. Ghannouchi, and B. Vassilakis, "Digital predistorter architecture with small signal gain control for highly nonlinear RF power amplifiers," 2007 50th Midwest Symposium on Circuits and Systems, pp. 12011204, Aug. 2007.

[37] A. Zerguine, O. Hammi, A. H. Abdelhafiz, M. Helaoui, and F. Ghannouchi, "Behavioral modeling and predistortion of nonlinear power amplifiers based on adaptive filtering techniques," in 2014 IEEE 11th International Multi-Conference on Systems, Signals \&6 Devices (SSD14), pp. 1-5, IEEE, Feb. 2014.

[38] O. Hammi, S. Boumaiza, and F. M. Ghannouchi, "On the Robustness of Digital Predistortion Function Synthesis and Average Power Tracking for Highly Nonlinear Power Amplifiers," IEEE Transactions on Microwave Theory and Techniques, vol. 55, pp. 1382-1389, June 2007.

[39] J. Pedro and S. Maas, "A comparative overview of microwave and wireless poweramplifier behavioral modeling approaches," IEEE Transactions on Microwave Theory and Techniques, vol. 53, pp. 1150-1163, Apr. 2005. 
[40] W.-J. Kim, K.-J. Cho, S. Stapleton, and J.-H. Kim, "Piecewise Pre-Equalized Linearization of the Wireless Transmitter With a Doherty Amplifier," IEEE Transactions on Microwave Theory and Techniques, vol. 54, pp. 3469-3478, Sept. 2006.

[41] R. Braithwaite, "A Self-Generating Coefficient List for Machine Learning in RF Power Amplifiers using Adaptive Predistortion," in 2006 European Microwave Conference, pp. 1229-1232, IEEE, Sept. 2006.

[42] O. Hammi, A. Kwan, and F. M. Ghannouchi, "Bandwidth and Power Scalable Digital Predistorter for Compensating Dynamic Distortions in RF Power Amplifiers," IEEE Transactions on Broadcasting, vol. 59, pp. 520-527, Sept. 2013.

[43] A. Zhu, "Power adaptive digital predistortion for RF power amplifiers," in 2014 IEEE MTT-S International Microwave Symposium (IMS2014), pp. 1-3, IEEE, June 2014.

[44] K. Narendra, "Adaptive control of discrete-time systems using multiple models," IEEE Transactions on Automatic Control, vol. 45, no. 9, pp. 1669-1686, 2000.

[45] K. S. Narendra and O. A. Driollet, "Stochastic adaptive control using multiple models for improved performance in the presence of random disturbances," International Journal of Adaptive Control and Signal Processing, vol. 15, pp. 287-317, May 2001.

[46] K. S. Narendra, "New Concepts in Adaptive Control Using Multiple Models," IEEE Transactions on Automatic Control, vol. 57, pp. 78-89, Jan. 2012.

[47] A. Campbell and H. Schwartz, "Stochastic multiple model adaptive control with hypothesis testing," in Proceedings of the 2005, American Control Conference, 2005., pp. 366-367, IEEE, 2005.

[48] A. Campbell, Improvements to stochastic multiple model adaptive control: hypothesis test switching and a modified model arrangement. $\mathrm{PhD}$ thesis, Carleton University, Canada, 2005.

[49] A. S. Campbell and H. M. Schwartz, "Multiple model control improvements: hypothesis testing and modified model arrangement," Control and Intelligent Systems, vol. 35, pp. 236-243, June 2007. 
[50] T. Autenrieth and E. Rogers, "Performance enhancements for a class of multiple model adaptive control schemes," International Journal of Adaptive Control and Signal Processing, vol. 13, pp. 105-127, Mar. 1999.

[51] T. Liu, Y. Ye, X. Zeng, and F. M. Ghannouchi, "Accurate Time-Delay Estimation and Alignment for RF Power Amplifier/Transmitter Characterization," in 2008 4th IEEE International Conference on Circuits and Systems for Communications, pp. 70-74, IEEE, May 2008.

[52] C. Nader, P. N. Landin, W. Van Moer, N. Bjorsell, P. Handel, and D. Ronnow, "Peak-power controlling technique for enhancing digital pre-distortion of RF power amplifiers," IEEE Transactions on Microwave Theory and Techniques, vol. 60 , no. 11, pp. 3571-3581, 2012.

[53] M. Helaoui, S. Boumaiza, A. Ghazel, and F. Ghannouchi, "Power and efficiency enhancement of $3 \mathrm{G}$ multicarrier amplifiers using digital signal processing with experimental validation," IEEE Transactions on Microwave Theory and Techniques, vol. 54, pp. 1396-1404, June 2006.

[54] D. Lopez, P. L. Gilabert, G. Montero, and N. Bartzoudis, "Peak cancellation and digital predistortion of high-order QAM wideband signals for next generation wireless backhaul equipment," in 2014 International Workshop on Integrated Nonlinear Microwave and Millimetre-wave Circuits (INMMiC), pp. 1-3, IEEE, Apr. 2014.

[55] L. Guan and A. Zhu, "Optimized low-complexity implementation of least squares based model extraction for digital predistortion of RF power amplifiers," IEEE Transactions on Microwave Theory and Techniques, vol. 60, no. 3 PART 1, pp. 594-603, 2012.

[56] H. Qian, S. Yao, H. Huang, and W. Feng, "A Low-Complexity Digital Predistortion Algorithm for Power Amplifier Linearization," IEEE Transactions on Broadcasting, vol. 60, pp. 670-678, Dec. 2014. 